Portland State University

PDXScholar

$1-1-2012$

\title{
Examining the Effect of Maternal High-Fat Diet Consumption on the Physiology and Pancreas Development of Fetal and Juvenile Nonhuman Primate Offspring
}

Sarah Michelle Comstock

Portland State University

Follow this and additional works at: https://pdxscholar.library.pdx.edu/open_access_etds Let us know how access to this document benefits you.

\section{Recommended Citation}

Comstock, Sarah Michelle, "Examining the Effect of Maternal High-Fat Diet Consumption on the Physiology and Pancreas Development of Fetal and Juvenile Nonhuman Primate Offspring" (2012). Dissertations and Theses. Paper 551.

https://doi.org/10.15760/etd.551

This Dissertation is brought to you for free and open access. It has been accepted for inclusion in Dissertations and Theses by an authorized administrator of PDXScholar. Please contact us if we can make this document more accessible: pdxscholar@pdx.edu. 
Examining the Effect of Maternal High-Fat Diet Consumption on the Physiology and Pancreas Development of Fetal and Juvenile Nonhuman Primate Offspring

by

Sarah Michelle Comstock

A dissertation submitted in partial fulfillment of the requirements for the degree of

\author{
Doctor of Philosophy \\ in \\ Biology \\ Dissertation Committee: \\ Keith Garlid, Chair \\ Brad Buckley \\ Kevin Grove \\ Jason Podrabsky \\ Charles Roberts \\ David Peyton
}

Portland State University

(C)2012 


\begin{abstract}
The purpose of these studies was to investigate the impact of high-fat diet (HFD) exposure during pregnancy and the early post-natal period on fetal and post-natal development of the endocrine pancreas of the Japanese macaque. Specifically I hypothesized that the HFD would alter islet morphology and lead to disturbances in glucose homeostasis in these animals.
\end{abstract}

Adult female Japanese macaques were placed on either a control (CTR) or HFD diet for 4 years. Fetuses were collected at gestational day 130 (G130), while other offspring from the CTR and HFD mothers were carried to term. After birth, infant animals were maintained with their mothers on the same diet then weaned onto either the CTR or HFD diet for five months. Animals were studied up to 13 months of age, yielding 4 postnatal groups: CTR/CTR, CTR/HFD, HFD/CTR and HFD/HFD. Pancreata were collected from these offspring for gene expression and immunohistochemical analysis. Physiological measurements, including body weight, body fat percentage, fasting glucose, insulin, glucagon and response to intravenous glucose tolerance tests (IVGTTs) and an intravenous insulin tolerance test (IVITT) were collected from the post-natal offspring.

Total fetal islet mass and $\beta$ cell mass were not changed, but $\alpha$ cell mass was significantly decreased in HFD fetuses, leading to a significant increase in the $\beta$ cell to $\alpha$ cell ratio in HFD fetal offspring. The HFD offspring displayed a significant change from CTR offspring in expression of genes involved in glucose homeostasis and islet neogenesis, including PDX1, NeuroD, Glucokinase and Glut2. Postnatal HFD animals were significantly heavier than CTR offspring and had increased adiposity by 6-7 months Comstock, i 
of age. There was no significant effect on fasting or stimulated insulin secretion at this time point, but HFD offspring were significantly insulin resistant just prior to weaning.

At 13 months of age, basal and glucose-stimulated insulin secretion were elevated in HFD/HFD animals and the CTR/HFD group displayed moderate insulin resistance. There was also a significant sex effect, with males from the HFD/CTR and HFD/HFD group having increased body weight and elevated fasting glucose. Although pancreata from both the HFD/HFD and CTR/HFD animals displayed significant changes in expression of genes involved in glucose homeostasis, the pattern was distinct for the two groups. Islet mass was also elevated in both of these groups; yet, HFD/HFD only displayed an increase in $\beta$ cell area, while CTR/HFD had a concomitant increase in $\alpha$ cell area, which served to normalize the $\beta$ cell to $\alpha$ cell ratio to control levels. In contrast, the HFD/HFD group exhibited a $40 \%$ increase in the $\beta$ cell to $\alpha$ cell ratio.

These studies demonstrate that in-utero exposure to a HFD leads to decreased $\alpha$ cell plasticity in response to chronic post-natal HFD consumption. Animals exposed to the HFD during pregnancy and the early post-natal period become insulin resistant, but remain normoglycemic. HFD consumption during the post-weaning period causes similar complications in glucose homeostasis and islet mass in both the CTR/HFD and HFD/HFD animals. However, there are distinct differences in the molecular and cellular adaptive response between these two groups. 


\title{
Dedication
}

\author{
To my husband, Ben Comstock \\ You are my rock and encouragement. \\ Thank you for your love and support.
}

\section{To my son, Droiy Comstock}

You are my inspiration and my joy.

Thank you for your love and enthusiasm.

Comstock, iii 


\section{Acknowledgments}

I would like to acknowledge and thank all those who have helped make this research possible. First I am grateful to the members of my committee. Thank you, Dr. Kevin Grove for mentoring me and allowing me to complete my research in your lab. Thank you, Dr. Keith Garlid for your willingness to support me in the accomplishment of this research. Thank you to the other members of my committee, Dr. Bradley Buckley, Dr. Jason Podrabsky, Dr. David Peyton and Dr. Charles Roberts, for being supportive and flexible.

I am grateful to the members of my lab. Thank you, Anne Evans for introducing me to laboratory research and for being a supportive friend through both the fortunate and difficult chapters of the past 10 years. Thank you, Diana Takahashi for taking over the NHP projects and being a gracious manager and friend. I am grateful to the members of the Gestational Diabetes Mellitus Team and the Obese Resource Team from the Grove Laboratory who are dedicated to the care of our animals.

I also want to acknowledge and thank the technical assistance of many at the Oregon National Primate Research Center, including the Division of Animal Resources, the Veterinary Staff, the RIA Core, and the Imaging Core.

I am also grateful to the members of my family for their support and encouragement throughout the completion of my $\mathrm{PhD}$ research. Thank you, Gary and Julie Williams, Tim and Sandra Joachim, Alex Williams and Jeff, Maureen and Jenny Comstock for your love and support. 


\section{Table of Contents}

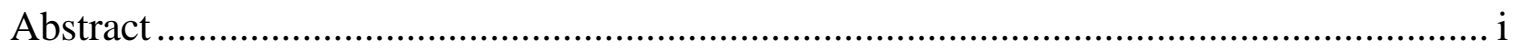

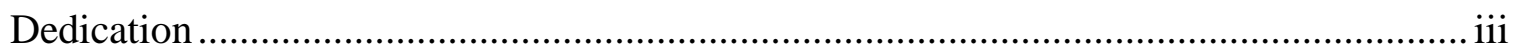

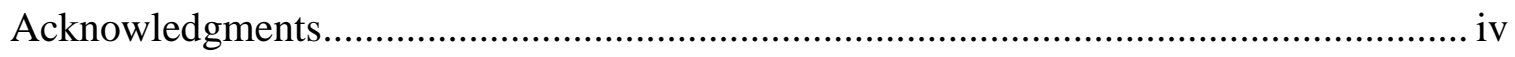

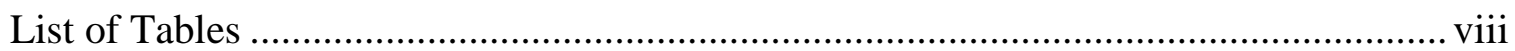

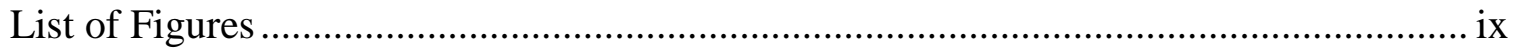

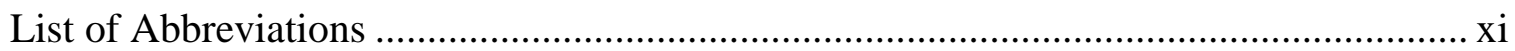

Chapter 1: Background and Introduction...................................................................... 1

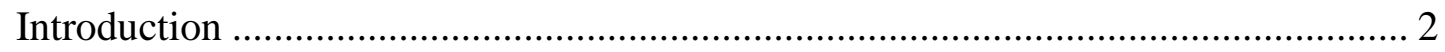

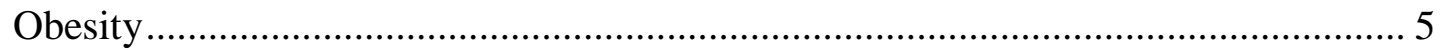

Homeostasis ........................................................................................... 7

Pancreas Morphology, Function and Development ........................................... 9

Type 2 Diabetes Mellitus and the Glucolipotoxicity Hypothesis ............................ 14

Animal Models of Maternal Overnutrition............................................................ 17

Animal Models of Intrauterine Growth Restriction ............................................. 20

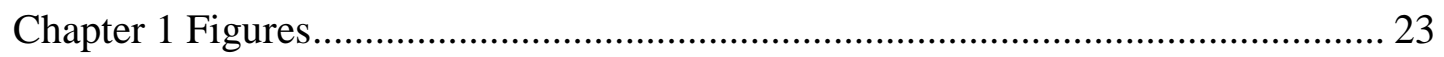

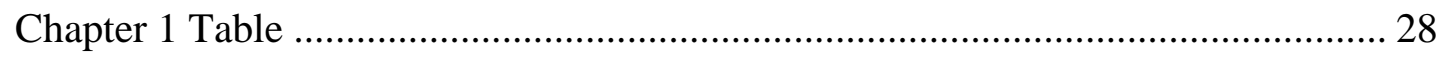

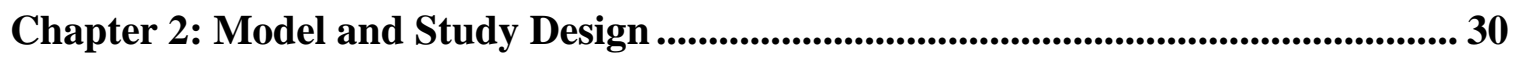

A Nonhuman Primate Model of Maternal HFD Consumption .............................. 31

Metabolic Syndrome and Type 2 Diabetes Mellitus in the Macaque....................... 35

Comstock, v 


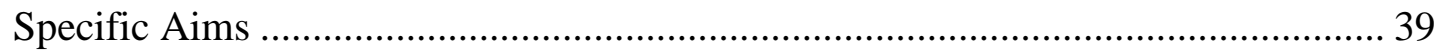

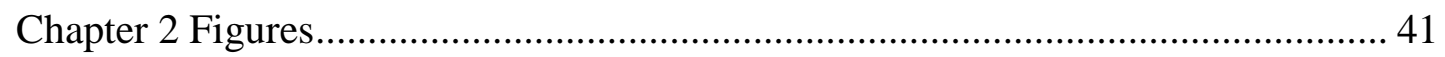

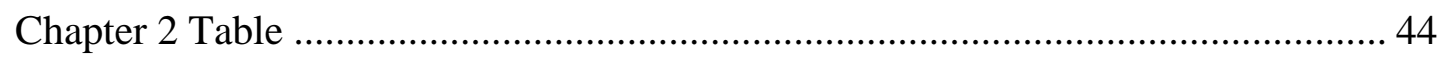

\section{Chapter 3: Maternal High-fat Diet Consumption During Pregnancy Leads to} Reduced $\alpha$ Cell Mass in Fetal Offspring ......................................................................... 46

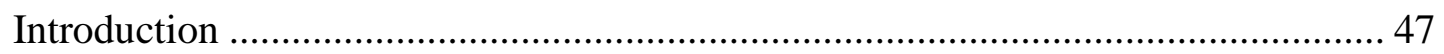

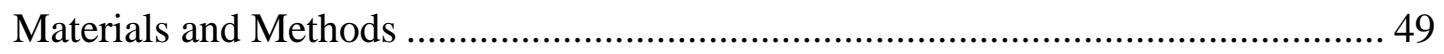

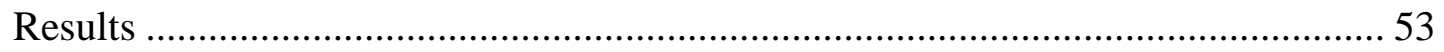

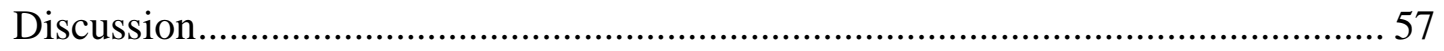

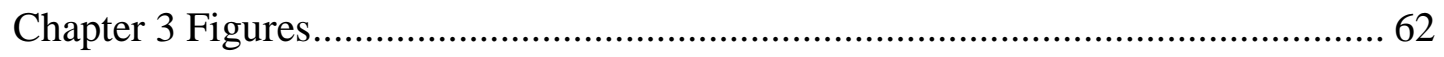

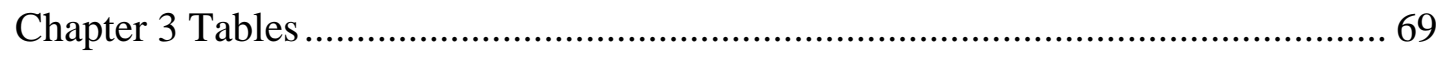

\section{Chapter 4: High-Fat Diet During Perinatal Development Leads to Early Insulin}

Resistance in the Nonhuman Primate Infant ...................................................... 73

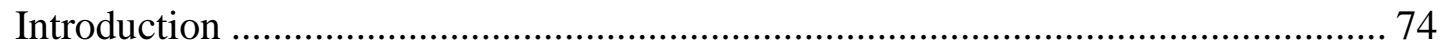

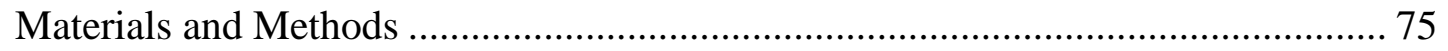

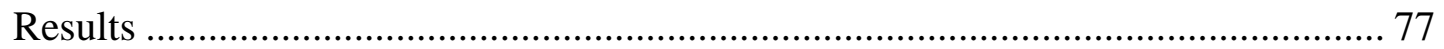

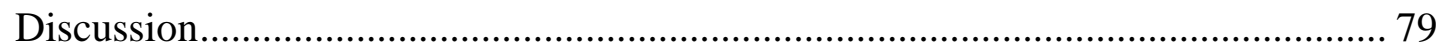

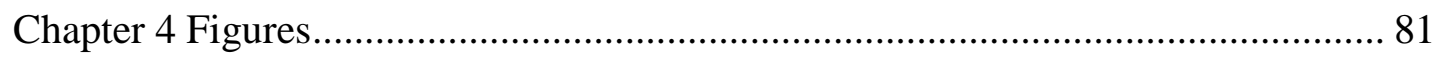

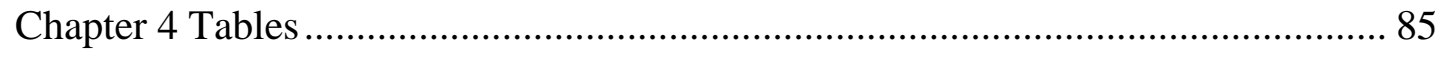


Chapter 5: Chronic High-Fat Diet Consumption During Pregnancy and the Early Postnatal Period Leads to Decreased $\alpha$ Cell Plasticity in the Juvenile Nonhuman

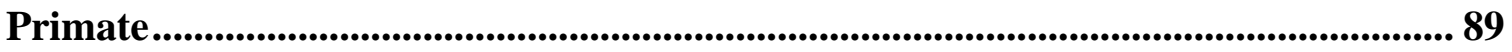

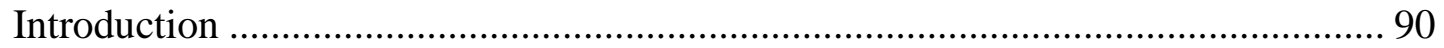

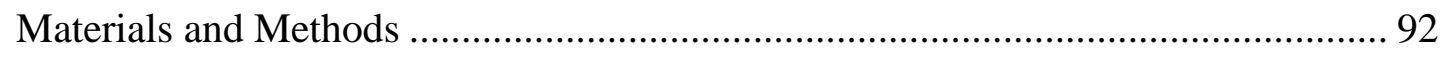

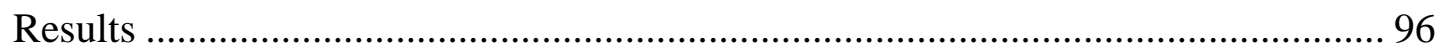

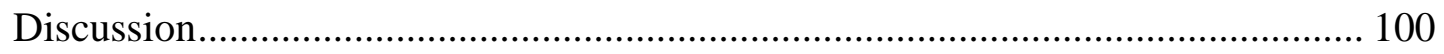

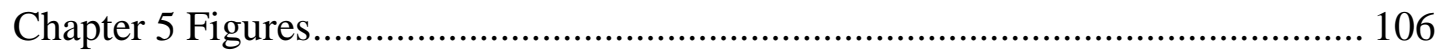

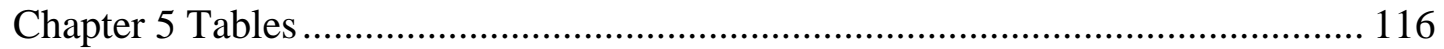

Chapter 6: Limitations and Future Studies............................................................................ 122

Limitations of the HFD Fed Macaque Model ......................................................... 123

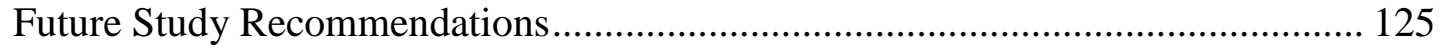

Chapter 7: Summary and Discussion....................................................................................... 130

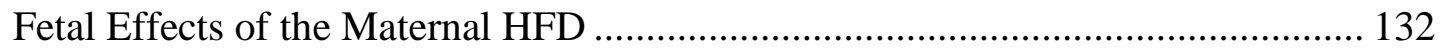

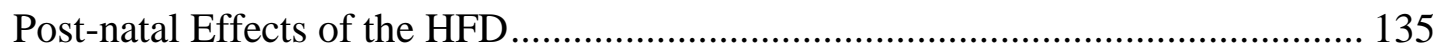

Chapter 7 Figures.............................................................................................. 138

Works Cited................................................................................................................................. 143 


\section{List of Tables}

Table 1.1: Embryonic/Fetal Development of the Pancreas in Macaques and Humans .... 29

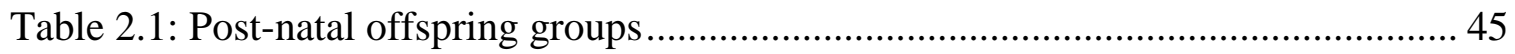

Table 3.1: Comparison of CTR and HFD Fetuses ........................................................... 70

Table 3.2: Real-time RT-PCR Gene Expression in Fetal Pancreas ................................... 71

Table 3.3: Comparison of CTR and HFD Dams...................................................... 72

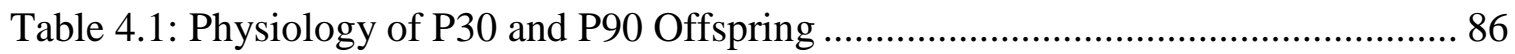

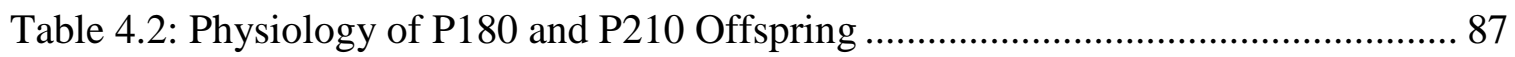

Table 4.3: Physiology of Dams of Infant Offspring ………….................................... 88

Table 5.1: Juvenile Physiology …………………………........................................ 117

Table 5.2: Juvenile Female Physiology ................................................................. 118

Table 5.3: Juvenile Male Physiology ................................................................... 119

Table 5.4: Real-time PCR Values of Juvenile Pancreas ................................................. 120

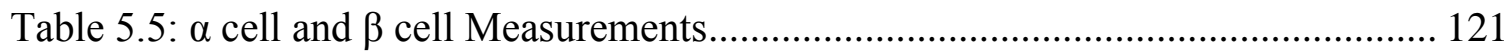




\section{List of Figures}

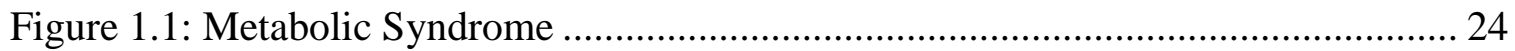

Figure 1.2: Diagram of Glucose Homeostasis ....................................................... 25

Figure 1.3: Diagram of Fully Formed Pancreas....................................................... 26

Figure 1.4: Glucose-stimulated Insulin Secretion in the $\beta$ cell ................................. 27

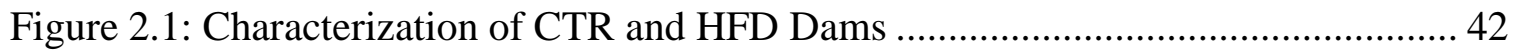

Figure 2.2: Characterization of Metabolic Syndrome and Diabetes in Females .............. 43

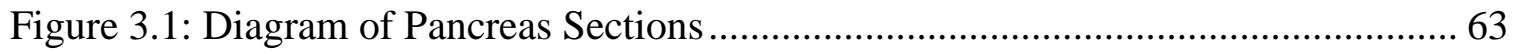

Figure 3.2: Immunohistochemical Analysis of Fetal Islet Mass................................... 64

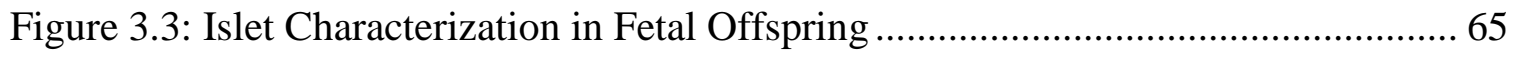

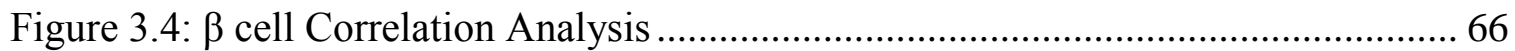

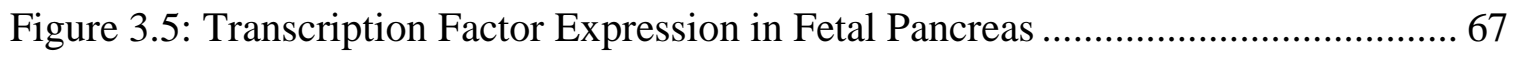

Figure 3.6: Expression of Genes Involved in Glucose-stimulated Insulin Secretion in

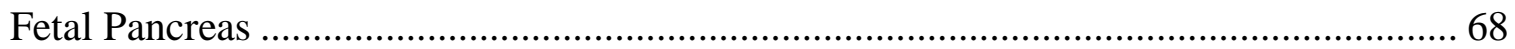

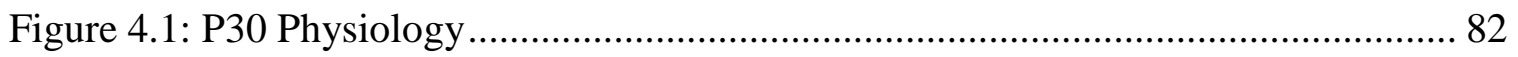

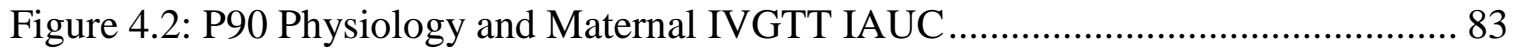

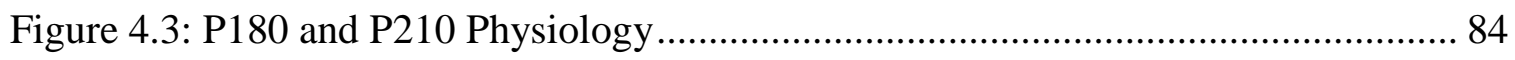

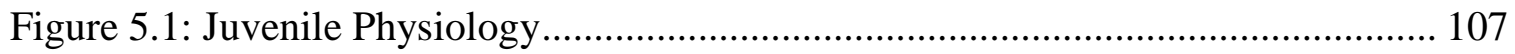

Figure 5.2: Sex Differences in Body Weight and Fasting Glucose ............................ 108

Figure 5.3: Juvenile Islet Mass and Contributing Factors ....................................... 109

Figure 5.4: Relative Mass, Diameter and Density of Islets ...................................... 110

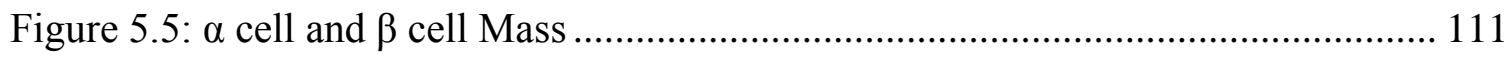

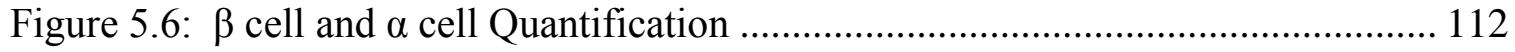

Comstock, ix 
Figure 5.7: Gene Expression in Juvenile Pancreas .................................................. 113

Figure 5.8: Normalized Gene expression in Juvenile Pancreas ................................... 114

Figure 5.9: Glut2 versus Glut1 Expression........................................................ 115

Figure 7.1: Islet Development in the CTR versus HFD Offspring .............................. 139

Figure 7.2: Islet Paracrine Function - Glucagon Regulation of Insulin Secretion ......... 140

Figure 7.3: Islet Paracrine Function - Insulin Regulation of Glucagon Secretion ......... 141

Figure 7.4: Pathological Progression to T2DM ....................................................... 142 


\section{List of Abbreviations}

Cldu - 5-chlorodeoxyuridine

$\mathrm{CNS}$ - central nervous system

CTR - control

CTR/CTR - postnatal offspring born to a mother on a CTR diet and maintained on this diet until necropsy at 13 months of age.

CTR/HFD - postnatal offspring born to a mother on a CTR diet and switched to the HFD after weaning at approximately 8 months of age.

CVD - cardiovascular disease

DHA - docosahexaenoic acid

FFA - free fatty acid

G130 -fetus collected by c-section at 130 days gestation

GIP - gastric inhibitory peptide

GK - glucokinase

GLP-1 - glucagon like peptide-1

GLUT1 - facilitated glucose transporter 1

Comstock, xi 
GLUT2 - faciltiated glucose transporter 2

GLUT5 - facilitated glucose transporter 5

GR - glucagon receptor

GSIS - glucose-stimulated insulin secretion

HFD - high-fat diet

HFD/CTR - postnatal offspring born to a mother on a HFD and switched to the CTR diet after weaning at approximately 8 months of age

HFD/HFD - postnatal offspring born to a mother on a HFD and maintained on this diet until necropsy at 13 months of age.

HGP - hepatic glucose production

HNF4 $\alpha$ - hepatocyte nuclear factor 4 alpha is a transcription factor that facilitates KCNJ11 transcription

IAPP - islet amyloid polypeptide

Idu - 5-iododeoxyuridine

INGAP - islet neogenesis-associated protein

IR - insulin receptor 
IUGR - intra-uterine growth restriction

IVGTT - intravenous glucose tolerance test

IVITT - intravenous insulin tolerance test

KATP - potassium sensitive ATP channel

KCNJ11 - gene encoding for Kir6.2

Kir6.2 - inward rectifier K+ channel subunit of the KATP channel in islet cells

MafA - musculoaponeurotic fibrosarcoma oncogene homolog A

MetS - metabolic syndrome

NAFLD - non-alcoholic fatty liver disease

$\mathrm{NHP}$ - nonhuman primate

NKX6.1 - NK6 homeobox 1 is a transcription factor expressed in $\beta$ cells

NeuroD - also called BETA2 is a transcription factor expressed in islet cells

P30 - postnatal day 30 in NHP offspring

P90 - postnatal day 90 in NHP offspring

P180 - postnatal day 180 in NHP offspring 
P210 - postnatal day 210 in NHP offspring

PCR - polymerase chain reaction

PCK1 - phosphoenolpyruvate carboxykinase 1 is a transcription factor involved in hepatic gluconeogenesis

PDX1 - pancreatic duodenal homeobox 1 is a transcription factor expressed in the pancreas

PGC1A - peroxisome proliferator-activated receptor gamma coactivator-1 alpha is a transcription factor involved in hepatic gluconeogenesis

PUFA - polyunsaturated fatty acid

qRT-PCR - quantitative reverse-transcription PCR

SUR1 - sulfonylurea receptor 1subcomponent of the KATP channel in islet cells

T2DM - Type 2 Diabetes Mellitus

TG - triglyceride

TUNEL - terminal deoxynucleotidyl transferase dUTP nick end labeling 
Chapter 1: Background and Introduction

Comstock, 1 


\section{Introduction}

The framework of my research is based on the theory that environmental stimuli during fetal development lead to adaptations in the fetus to prepare it for post-natal life conditions. This concept was first presented by Dr. David Barker, a physician and professor of clinical epidemiology at the University of Southampton and a professor in the Department of Cardiovascular Medicine at Oregon Health and Science University. Based on his research in epidemiology, Dr. Barker presented the now widely accepted theory that disturbed intrauterine growth has a negative influence on fetal development

which leads to an increased risk for multiple diseases later in life, such as Type 2 Diabetes Mellitus (T2DM) and cardiovascular disease (CVD) (Barker, Winter, Osmond, Margetts, \& Simmonds, 1989; Hales \& Barker, 1992). During fetal development, the inutero environment is significantly affected by maternal health and nutrition. The conditions at this stage provide a signal to the fetus of what to expect after birth. The fetus often adapts to early life stress, such as malnourishment, by trading off the development of less critical organs, such as the pancreas, in favor of maintaining the development of essential organs such as the brain. These adaptations allow the fetus to survive until birth while maintaining critical organs that are essential for post-natal survival. However, poor development of less essential organs usually leads to significant health complications during later life.

Many studies on humans and animals support this hypothesis. However, a unique human population, composed of adult children born to mothers who were pregnant during the Dutch famine, provides distinctive insight into the link between fetal 
undernutrition and adverse post-natal health effects (Smith, 1947a). This famine lasted from November 1944 to May 1945 due to an exhaustion of food supplies during World War II in the western portion of the Netherlands. At the height of the famine, official rations fell to between 400 and 800 calories per day. While pregnant and lactating women were entitled to additional food, supplies were insufficient to accommodate this need. However, when the Netherlands was liberated in May 1945, food rations rose nearly immediately to over 2000 calories per day (T. Roseboom, de Rooij, \& Painter, 2006). Studies performed on survivors of this famine demonstrated that babies born during the famine were growth restricted, weighing about 200 grams lighter at birth than those born to the same mothers not during the famine (Smith, 1947b). Offspring during their adult life were also found to have an increased risk for developing T2DM and CVD (Ravelli et al., 1998; T. J. Roseboom et al., 2000). The health complications observed in the Dutch famine adult offspring demonstrate a significant effect of fetal malnourishment on adult health outcomes. Based on this and many other studies of fetal intra-uterine growth restriction (IUGR), Dr. Barker hypothesized that fetuses that undergo IUGR develop a "thrifty phenotype," which makes them more likely to conserve energy and become obese later in life. The early life undernutrition permanently changes glucoseinsulin metabolism which leads to insulin resistance and reduced insulin secretion (Phillips, 1996). This ultimately leads to an increased incidence of obesity and T2DM within this population (Hales \& Barker, 1992).

Although fetal malnoushiment may be an expected concern in developing countries, where many young women of child-bearing years are chronically under- 
nourished, it is also a concern in Western countries, such as those in North America and the Europen Union. It is important to understand that both maternal undernutrition and overnutrition can result in fetal malnourishment, when inappropriate nutrients are delivered to the fetus. For example, in the case of overnutrition, maternal consumption of high calorie diets has been demonstrated to lead to fetal malnoushishment because these diets are unbalanced in macronutrients and deficient in micronutrients necessary for normal fetal development (Shafrir \& Khassis, 1982). These diets are usually rich in carbohydrates and saturated fat, but deficient in protein and essential vitamins, which directly affect fetal nutrient availability (Rao et al., 2001; Villar et al., 2003). Also, the diets have a direct impact on the health of the mother, because consumption of a high calorie diet usually leads to the development of obesity. Maternal obesity can greatly affect the nutrient supply to the fetus because placental function is significantly affected if a mother is excessively overweight (Farley et al., 2009; Radaelli et al., 2009; Varastehpour et al., 2006). The affect on the placenta often translates to fetal IUGR because the complications of obesity result in fetal malnourishment. Maternal obesity greatly increases the risk of obesity in their offspring. It is necessary to understand how the unbalanced or restricted nutrient supply from these mothers affects the developing fetus. My research focuses on how maternal obesity and the consumption of a highcalorie/high-fat diet may affect the developing offspring. 


\section{Obesity}

Obesity is a global epidemic. In fact, approximately $20 \%$ of the world's population is classified as either obese or overweight (Finucane et al.). However, in the United States, the world leader in this health crisis, more than one third of adults are obese, while an additional one third are classified as overweight (Ogden et al., 2006). While obesity is a serious health concern, the added burden of the co-morbidities associated with obesity, including T2DM and CVD, make this disease a significant health burden (Burton, Foster, Hirsch, \& Van Itallie, 1985). In fact, a classification termed Metabolic Syndrome (MetS) has been designated to a cluster of metabolic risk factors that usually appear together and directly promote the development of both CVD and T2DM (Grundy et al., 2005). These factors include central obesity, elevated triglycerides, reduced HDL cholesterol, elevated blood pressure and elevated fasting glucose (Alberti et al., 2009) (Fig. 1.2). While some health organizations encourage treating the symptoms individually and discourage this classification (R. Kahn, Buse, Ferrannini, \& Stern, 2005), the coexistence of these disorders appears to be more frequent than would be expected by chance (Grundy, 2006). In addition, the clustering of these symptoms increase the risk for CVD and T2DM above the effect of individual risk factors. Moreover, increasing evidence indicates that insulin resistance may be the common etiological factor that is leading to these symptoms (Haffner et al., 1992; Zimmet, 1992).

An additional confounding factor that adds to the obesity crisis is children born to obese mothers (Boney, Verma, Tucker, \& Vohr, 2005; Forsen et al., 1997; Koupil \& 
Toivanen, 2008; Whitaker, 2004) or those who consume a high-fat diet (HFD) during pregnancy (Bayol, Simbi, \& Stickland, 2005; Buckley et al., 2005) are at increased risk for childhood obesity and MetS. This is evidenced by the steep rise in childhood obesity that has followed the increase in adult obesity within the last few decades. In fact, today approximately $17 \%$ of children in the United States are classified as obese, while an additional 14\% are overweight (Ogden, Carroll, Curtin, Lamb, \& Flegal, 2010). Childhood obesity is becoming an epidemic that has far reaching effects in most modern societies. Likewise, the International Obesity Task Force categorizes over $20 \%$ of children in the European Union as being overweight or obese (Cattaneo et al., 2010). Since early childhood and adolescent obesity is a strong predictor of being obese or overweight as an adult (Guo, Wu, Chumlea, \& Roche, 2002; Magarey, Daniels, Boulton, \& Cockington, 2003), this rise in childhood obesity indicates that we have not reached the peak in the obesity health crisis that is plaguing our world today. If the incidence of obesity continues to rise, especially among women, we will likely see an increased incidence of childhood MetS in future generations. It is necessary to understand the mechanisms that lead to the increased risk for childhood obesity and MetS in order to treat or prevent this disease.

Comstock, 6 


\section{Homeostasis}

In order to understand the disease progression of obesity and MetS, it is essential to keep in mind the normal homeostatic mechanisms that are disregulated by these health issues. As I indicated earlier, many factors are pushing the scale toward increased energy input while minimizing energy output. However, the healthy individual maintains a normal, steady weight due to a balance between signals from both the central nervous system (CNS) and peripheral organs. There is complex interplay between peripheral hormone signaling and CNS processing of these signals.

A important homeostatic mechanism is the regulation of whole-body glucose homeostasis. Glucose is an essential fuel source for the body, that, when broken down within individual cells, provides sufficient energy to support most cellular processes (Dzeja \& Terzic, 2003; Dzeja, Zeleznikar, \& Goldberg, 1998). The circulating levels of glucose within a healthy individual are tightly controlled by counterregulatory hormones. In fact, after a meal, when circulating glucose levels rise, insulin is released by the pancreas and leads to glucose uptake and utilization by various organs, which, in turn, reduces the level of glucose in the circulation (Cushman \& Wardzala, 1980; Lang, Goldstein, \& Levine, 1954). However, this uptake is counterbalanced by glucagon that is released from the pancreas, when glucose levels fall, which results in the generation and release of glucose back into circulation (Drury, Wick, Bancroft, \& Mac, 1951; Staub, Sinn, \& Behrens, 1955) (Fig. 1.3). In a healthy individual, these hormones tightly control glucose levels to meet the body's requirements. However, disregulation of these hormones can lead to obesity and T2DM. It is essential to understand the normal 
function of the pancreas in order to determine how disregulation of insulin and glucagon can lead to these diseases. In the next section, I will explain pancreas development and morphology and the role of this organ in maintaining glucose homeostasis.

Comstock, 8 


\section{Pancreas Morphology, Function and Development}

The pancreas is both an exocrine and an endocrine organ. The exocrine tissue is composed of ductal cells that secrete bicarbonate $\left(\mathrm{HCO}_{3}{ }^{-}\right)$, which acts to neutralize stomach chyme that is delivered to the duodenum (Hegyi, Maleth, Venglovecz, \& Rakonczay, 2011), and also acinar cells that secrete digestive enzymes, which aid in the digestion of protein, fat and carbohydrates within the intestine (Carriere, Barrowman, Verger, \& Laugier, 1993; Williams \& Lee, 1976). The endocrine pancreas is made up of islets of Langerhans, which are composed of four main cell types that secrete hormones directly into the circulatory system. In humans and the the nonhuman primate (NHP), $\beta$ cells, which are the most common islet cell type (about 50-70\%), secrete insulin and amylin (Cabrera et al., 2006). The $\alpha$ cells make up 30-45\% of the total islets and secrete glucagon, glucagon like peptide (GLP1), gastric inhibitory peptide (GIP) and (Cabrera, et al., 2006). Less common are the $\delta$ cells and the PP cells, which secrete somatostatin and pancreatic polypeptide respectively (Ganong, 2007; Hall, 2000). In the rodent, a commonly used model for studying islet morphology, the $\beta$ cells form a core at the center of the islet and are surrounded by $\alpha, \delta$ and PP cells. However, the NHP islet, the model I use for research, is more similar to the human islet, with a more random organization but with clear associations with other endocrine cells, suggesting unique paracrine interactions in islets of both humans and NHPs (Brissova et al., 2005; Cabrera, et al., 2006; Kim et al., 2009). This makes the NHP an excellent model for the study of islet development and function that is relevant to the human condition. 
It should be noted that pancreas development follows a similar pattern in mammals. The key developmental occurrences are indicated in Table 1.1 which is derived from studies in humans and macaques (Fowden \& Hill, 2001; Gerich, 1976; Hill, 2005; Kerr, Allen, Scheffler, \& Couture, 1974; Myers et al., 1971; Piper et al., 2004; Remacle et al., 2007). In summary, within the embryo, the pancreas develops from two buds that form as an outgrowth from the primitive gut - termed the dorsal and ventral buds. In the human and the macaque, the dorsal bud first appears less than a month after conception; then the ventral bud appears in the embryo about 24 hours later. Ultimately the ventral bud rotates around the stomach and fuses with the dorsal bud, forming one organ. By the middle of the first trimester, the pancreas resembles the adult organ with a head, body, tail and an uncinate process (Fig. 1.4). Endocrine cells and acinar cells develop from ductal cell precursors. These cells bud off to form clumps of acinar cells or endocrine cells, which differentiate into individual islet cells (Fowden \& Hill, 2001; Piper, et al., 2004; Remacle, et al., 2007). By the beginning of the second trimester, mature islets are noticable, based on the accumulation of islet amyloid polypeptide (IAPP) in $\alpha$ cells and $\beta$ cells (Lukinius, Korsgren, Grimelius, \& Wilander, 1996). Toward the end of the second trimester, most islets are fully functional and they begin to proliferate during the third trimester (Mourmeaux, Remacle, \& Henquin, 1985). This makes the third trimester a critical window of islet expansion. By birth, islets have been demonstrated to compose approximately $4 \%$ of the human pancreas (Fowden \& Hill, 2001). After birth, there is a significant period of islet remodeling that is marked by islet replication and neogenesis which is counter-balanced by apoptosis (Bonner-Weir, 2001; Georgia \& Bhushan, 2004; Scaglia, Cahill, Finegood, \& Bonner-Weir, 1997). However, 
the potential for replication appears to significantly decline with age, so that by adulthood, while the pancreas is significantly larger than it is at birth, islets now only compose 1 to $2 \%$ of its total mass. Within the healthy adult, islet mass is usually homeostatically maintained due to a balance between neogenesis and apoptosis (Butler et al., 2010; Hanley et al., 2010; Reers et al., 2009); however, some factors can induce islet loss or expansion. One such factor is obesity, which leads to islet expansion, principally due to the expansion of $\beta$ cells. A second phenomenon that results in islet size disturbance is insulin resistance, which initially leads to islet expansion but ultimately may result in $\beta$ cell loss if the disease progresses to T2DM (Butler et al., 2003). In order to determine the causes of islet cell destabilization, it is necessary to understand the function of these cells.

The role of the pancreas is critical in maintaining glucose homeostasis due, primarily to the function of $\beta$ cells and $\alpha$ cells. In healthy subjects, $\beta$ cells secrete insulin in response to elevated circulating glucose levels. Glucose uptake and insulin secretion by $\beta$ cells is a complex pathway (Fig. 1.5). The first step in this pathway is mediated by the facilitated glucose transporter 2 (GLUT2) which permits diffusion of glucose into the $\beta$ cell. Once glucose enters the cell, it is phosphorylated by hexokinase or glucokinase (GCK) into glucose-6-phosphate (G6P). GCK is considered a "glucose sensor," determining the threshold level of glucose necessary for insulin secretion (Postic, Shiota, \& Magnuson, 2001). G6P is then metabolized by the additional steps of glycolysis to produce pyruvate, NADH and ATP. NADH and pyruvate both enter the mitochondria where they are metabolized to produce an estimated $98 \%$ of the total $\beta$ cell ATP content 
(MacDonald, Joseph, \& Rorsman, 2005), resulting in the increase of cytosolic ATP/ADP ratio. ATP-sensitive potassium (KATP) channels on the cell membrane are closed as a result of the increased ATP/ADP ratio, leading to depolarization of the $\beta$ cells. These channels are composed of 2 subunits - the sulfonyurea receptor 1 (SUR1) and the inwardly rectifying potassium channel 6.2 (Kir6.2, encoded by the KCNJ11 mRNA). The depolarization of $\beta$ cells leads to the opening of voltage-dependent calcium channels that allow the influx of calcium. The rise in $\beta$ cell calcium concentration triggers insulin granule exocytosis (Miki, Nagashima, \& Seino, 1999). After it is released into circulation, insulin is bound by insulin receptors in various target tissues which leads to the uptake of glucose to be used as a fuel source or stored for later use.

However, when glucose levels start to fall, $\alpha$ cells secrete glucagon. It should be noted, however, that $\alpha$ cells also take up glucose via the glucose transporter Glut1, store it as glycogen and then later metabolize glycogen as a fuel source ATP production (Quesada, Tuduri, Ripoll, \& Nadal, 2008). Thus, while glucose inhibits glucagon secretion, glucose is also required for maintenance of $\alpha$ cell energetics. Little is known about the mechanism that leads to the stimulation or inhibition of glucagon secretion (Walker et al., 2011). However, recent studies indicate that glucagon secretion may be mediated by the effects of insulin or other $\beta$ cell secretory products on $\alpha$ cells. Elevated insulin levels have been shown to lead to sensitization of KATP channels within the $\alpha$ cell, which causes $\alpha$ cell hyperpolarization and inhibits glucagon release (Franklin, Gromada, Gjinovci, Theander, \& Wollheim, 2005; Leung et al., 2006). In contrast, when glucose levels decline the inhibitory signal produced by $\beta$ cell (i.e. insulin, zinc or 
GABA) is diminished. This results in decreased efflux of potassium through KATP channels, which when exposed to and alevated ATP/ADP ratio produced by glycogen metabolism, will lead to depolarization of $\alpha$ cells and glucagon secretion. When glucagon is elevated, it is taken up by hepatic cells which undergo glycogenolysis conversion of glycogen to glucose by the enzyme glucose-6-phosphatase - thereby releasing glucose into the bloodstream. When glycogen stores are depleted, hepatic cells produce glucose through a process called gluconeogenesis - anabolic converstion of noncarbohydrate sources, such as lactate and pyruvate, into glucose (Chakravarty, Cassuto, Reshef, \& Hanson, 2005; Consoli, Nurjhan, Capani, \& Gerich, 1989; Yoon et al., 2001). Glucagon also promotes lipolysis of adipose tissue, which is the breakdown of triglycerides from fat depots into FFAs and glycerol, by increasing the mobilization of glycerol after lipolysis occurs (Slavin, Ong, \& Kern, 1994). FFAs and glycerol can then be used as sources of energy for the body.

It is evident that healthy, functional islet cells work in concert to maintain glucose homeostasis. However, glucose homeostasis can be disrupted in obese individuals. This often leads to the development of MetS, which puts the individual at increased risk for developing T2DM. The mechanisms that lead to this disruption are intensely studied. In the next section I will explain the etiology of T2DM and the progression of MetS to the development of this disorder. I will also present the most widely accepted theory of the mechanism of T2DM disease progression. 


\section{Type 2 Diabetes Mellitus and the Glucolipotoxicity Hypothesis}

T2DM is a metabolic disorder that is characterized by elevated fasting glucose (hyperglycemia) that results in metabolic abnormalities due to defects in insulin action and insulin secretion. Diagnostic criteria for diabetes in adult humans is a fasting blood glucose greater than $126 \mathrm{mg} / \mathrm{dL}$ and a glucose level of $200 \mathrm{mg} / \mathrm{dL}$ or greater two hours after an oral glucose tolerance test ("Diagnosis and classification of diabetes mellitus," 2011). The etiology of T2DM is not completely understood, but the disease progression usually follows a similar pattern in animal models and humans. The risk for T2DM is associated with an increase in insulin levels and insulin resistance of peripheral tissues. This insulin resistance usually occurs concurrently with other maladies including central obesity and increased circulating triglycerides (TGs) which are hallmark symptoms of MetS. Ultimately T2DM will develop in $20 \%$ of MetS sufferers while the remaining $80 \%$ are able to compensate through $\beta$ cell expansion (E. S. Ford, Giles, \& Dietz, 2002; Guiot, Sempoux, Moulin, \& Rahier, 2001; L. C. Jones \& Clark, 2001). Many theories have been presented as what leads to T2DM progression. The most commonly accepted theory of the pathogenesis of this disease is that concurrent glucotoxicity and lipotoxicity of peripheral tissues, including pancreatic $\beta$ cells, leads to insulin resistance and ultimately progression to T2DM (Unger, 1995).

Glucotoxicity occurs when chronic hyperglycemia impairs glucose-stimulated insulin release of $\beta$ cells and reduces insulin gene transcription. $\beta$ cells become desensitized to glucose after a short exposure to elevated levels (above $100 \mathrm{mg} / \mathrm{dL}$ ), meaning they steadily release lower doses of insulin in response to the same glucose 
stimulus. This is a normal physiological adaptation that is readily reversible if glucose levels return to normal levels. However, if glucose is chronically elevated, $\beta$ cells are exhausted, meaning their releasable insulin pool is depleted (Kilpatrick \& Robertson, 1998). Initially this exhaustion is reversible. However, after long term, chronic hyperglycemia, exhaustion is no longer reversible and thus glucotoxicity ensues (Moran et al., 1997). Recent studies have shown that this chronic hyperglycemia leads to oxidative damage in $\beta$ cells that diminishes the activity of two insulin gene promoters PDX1 (pancreatic-duodenal homeobox 1) and MafA (musculoaponeurotic fibrosarcoma oncogene homolog A) - thereby reducing insulin gene transcription and insulin secretion (Olson et al., 1995; Robertson, 2004; Sharma, Olson, Robertson, \& Stein, 1995).

Lipotoxicity is a condition in which free fatty acids (FFAs) accumulate in excess of the required need of oxidative pathways within an organism and spill over into nonoxidative, toxic pathways (Unger \& Zhou, 2001). FFAs provide an alternative fuel source for the body during prolonged periods of fasting and protect against the use of proteins that would otherwise be used for gluconeogenesis - production of glucose by the liver. During the fed state, insulin is raised in order to use glucose as a fuel source and thereby suppresses circulating FFA levels. However, it has been shown that, in obese individuals and those who have MetS, FFA levels remain elevated in the presence of high insulin, likely because of an increase in adipose tissue and adipocyte insensitivity to insulin (Y. D. Chen, Golay, Swislocki, \& Reaven, 1987; Groop et al., 1992). The feedback between adipocytes and $\beta$ cells is thereby inhibited and thus leads to a paradoxical elevation of both basal insulin and FFAs.

Comstock, 15 
The glucolipotoxicity hypotheses postulates that chronically elevated glucose and FFAs impair $\beta$ cell function by inhibiting insulin transcription and secretion (Poitout, 2008). In patients that are susceptible to T2DM, there is a blunted response to nutrient overload caused by excess glucose and FFA (Cusi, Kashyap, Gastaldelli, Bajaj, \& Cersosimo, 2007; Kashyap et al., 2003). As indicated, this occurs prior to the development of the disease and may be a complicating factor that ultimately leads to disease progression. However, in patients with MetS, glucolipotoxocity leads to similar impairment of $\beta$ cell function, yet $80 \%$ of these patients do not develop overt diabetes. This disparity highlights the importance of investigating the causes that may lead to the differential development of T2DM within susceptible individuals.

While many factors may influence whether an individual ultimately develops T2DM, the early life environment likely plays a role in disease progression. While, both maternal HFD consumption and obesity increase the risk of developing MetS and T2DM in the offspring, it is unclear what mechanisms lead to this increased risk. Many others have investigated the effects of maternal HFD consumption or obesity on offspring and have provided insight into these mechanisms. These studies are reviewed in the following pages.

Comstock, 16 


\section{Animal Models of Maternal Overnutrition}

The most common animal models used to investigate HFD consumption and the effects of obesity are rodents - principally rats and mice. In fact, our lab has used both rats and mice as models of early post-natal overnutrition to study the effects of obesity on

metabolic systems (Enriori et al., 2007; Glavas et al., 2010; Xiao et al., 2007). By reducing litter size, our group is able to increase nutrient availability to young rodents. This results in increased susceptibility to obesity, insulin resistance and alterations in energy homeostasis, indicating that early overnutrition may increase the risk of MetS.

In order to study the effects of HFD on offspring, many studies in rodents provide the HFD to mothers during a portion of, or all of, gestation and/or lactation (Cerf, Muller, Du Toit, Louw, \& Wolfe-Coote, 2006; Cerf et al., 2005). However, the range of maternal HFD exposure in many rodent studies varies greatly - 21 days (for gestation only) up to 142 days (100 days prior to and including gestation and lactation). When dams consume a chronic HFD more than 30 days prior to conception, this usually results in maternal obesity - allowing some studies to focus on not only maternal HFD but also maternal obesity effects in the offspring (H. Chen, Simar, Lambert, Mercier, \& Morris, 2008; Ferezou-Viala et al., 2007; Howie, Sloboda, Kamal, \& Vickers, 2009; Srinivasan, Katewa, Palaniyappan, Pandya, \& Patel, 2006). The results of these studies vary based on the length of time the mother is exposed to the HFD. In most rodent models, the consumption of the HFD leads to MetS-like effects in the offspring: they become obese, hyperinsulemic, have a propensity to develop T2DM, and are at increased risk for 
developing CVD (Koukkou, Ghosh, Lowy, \& Poston, 1998; Srinivasan, et al., 2006; Taylor et al., 2005).

Specific effects on the pancreas of offspring exposed to maternal HFD have been described in a few of these models. In one study by Cerf et al., $\beta$ cell mass was reduced in 1-day-old rats only if the mother consumed a HFD all throughout pregnancy. Short-term exposure, for one week of the three week pregnancy, did not have a significant effect on $\beta$ cell mass, regardless of which week the mother consumed the HFD (Cerf, et al., 2005). When this study was followed through until the animals were of weaning age, offspring of mothers that were exposed to the diet through gestation and lactation were normoglycemic. However, when they were fed the HFD during lactation only, the offspring became hyperglycemic and hypoinsulinemic, indicating that switching from a healthy diet to a HFD may be more harmful than continued exposure to HFD alone (Cerf, et al., 2006).

While experimental models with sheep do not provide these animals a HFD, they are often used as a model of overnutrition by increasing their food supply above the recommended portion for their size (George et al., 2010; Zhu et al., 2008). These animals achieve obesity if allowed to consume an obesogenic diet (150\% of recommended calories) 60 days prior to conception. Differential results have been reported on developing islets from the fetuses of obese ewes based on the length of exposure to the diet. One study indicates that obese ewes produce fetuses at mid-gestation that are hyperglycemic, hyperinsulinemic, have over-growth of pancreatic islets and $\beta$ cell hyperplasia (S. P. Ford et al., 2009). However, if the fetuses were analyzed at the end of 
pregnancy, there was a significant reduction in pancreatic growth and markedly decreased numbers of $\beta$ cells due to increased apoptosis, which resulted in reduced circulating insulin concentrations in the fetuses from obese ewes (L. Zhang et al., 2011). One confounding factor of this research is that only ewes carrying singleton fetuses were analyzed in the mid-gestation study, whereas the fetuses that were carried to full term were twins. This makes the results difficult to interpret, because there is possibly increased growth restriction in the full term, twin sheep. Other studies in the sheep model of overnutrition have examined the effects maternal obesity on various organs, but do not describe how this affects the pancreatic islets (George, et al., 2010; Long et al., 2010; Zhu, et al., 2008).

The effects of maternal overnutrition on fetal development indicate that this is likely to cause MetS-like effects due to the increased adiposity, decreased insulin sensitivity and hyperinsulinemia demonstrated in these animals. However, it is unclear from these studies how pancreatic islets may be affected, since the reported results appear to conflict between the sheep and rodent models. It will be necessary to perform similar studies on a higher species, such as the NHP, that is more closely related to the human, in order to determine if factors within the endocrine pancreas may be leading to the association between maternal overnutrition and MetS in offspring. 


\section{Animal Models of Intrauterine Growth Restriction}

A second model which leads to obesity is intrauterine growth restriction (IUGR). Evidence from human studies has demonstrated that IUGR leads to an increased risk of developing MetS in adults (Barker, 1995; Barker et al., 1993). In fact, Dr. Barker's hypothesis of the developmental origins of adult disease theorizes that associations between poor fetal and infant growth and later development of T2DM and the MetS are a result of poor nutrition in early life (Hales \& Barker, 1992). IUGR is accomplished in rodent models through a variety of methods, including restricing maternal caloric or protein intake that both reduce the nutrient supply to the fetus. In addition, exposure to high levels of glucocorticoids or restriction of placental blood supply by bilateral uterine artery ligation both also lead to IUGR. These experimental models lead to decreased beta cell mass and lower pancreatic insulin content in neonatal offspring, yet ultimately these offspring become obese and diabetic (Dahri, Snoeck, Reusens-Billen, Remacle, \& Hoet, 1991; Dumortier et al., 2007; Garofano, Czernichow, \& Breant, 1997, 1998, 1999; Park, Stoffers, Nicholls, \& Simmons, 2008).

The ovine model of IUGR is accomplished by restricting maternal caloric intake, exposing pregnant dams to elevated temperatures $\left(40^{\circ} \mathrm{C}\right.$ versus $\left.25^{\circ} \mathrm{C}\right)$, induction of maternal hypoglycemia by continuous insulin infusion, uterine horn ligation, induction of uterine artery embolism or by surgical ablation of major uterine caruncles in the ewe prior to conception (Darp et al., 2010; Limesand, Jensen, Hutton, \& Hay, 2005; Meyer, Koch, Ramadoss, Kling, \& Magness, 2010; Morrison, 2008; Rozance, Limesand, \& Hay, 2006; Sebert et al., 2011). While all these models produce IUGR by different 
mechanisms, these protocols can result in smaller lambs that have deficient insulin secretion and usually smaller $\beta$ cells (Gatford et al., 2008; Limesand, Rozance, Zerbe, Hutton, \& Hay, 2006).

While studies in IUGR animals indicate that diabetes occurs due to the deficiencies in $\beta$ cell mass and insulin secretion, studies in human IUGR fetuses are not consistent with these findings. The human studies indicate that IUGR does not change fetal $\beta$ cell mass (Beringue et al., 2002), although adults with born with IUGR do have an increased risk for of developing T2DM, indicating that insulin resistance occurs first due to the insult of IUGR, followed by hyperinsulinemia. While the results from the IUGR animals may provide insight into islet development in animal models, they do not explain the association between human IUGR and deficiencies in insulin secretion. An animal model that may be more predictive of the effects of human IUGR would be the NHP. In fact, one recently described model of inter-placental vessel ligation that is being developed in rhesus macaques has the potential to lead to IUGR in its offspring (Roberts et al., 2012). However, no significant pancreatic fetal effects have been reported to date. Follow-up studies on these animals may provide insight into the mechanistic link between IUGR and insulin resistance.

Based on the experimental models described in the last two sections, it is evident that fetal malnutrition or early post-natal overnutrition may lead to the development of MetS-like effects and ultimately may increase the risk for T2DM, just as has been demonstrated in the human population. However, while the development of insulin resistance and hyperinsulinemia are common to all models, the effects on islets vary 
significantly. While some studies indicate that $\beta$ cell mass may be affected, no consistent effects on $\alpha$ cells have been studied or reported (Cerf, et al., 2005; S. P. Ford, et al., 2009; L. Zhang, et al., 2011). In addition, the inconsistencies between the described animal models and human studies make it evident that studies in higher species are necessary to determine the process that leads to the increased risk of MetS and T2DM in these offspring. For this reason, our lab has developed a NHP model of maternal HFD consumption and obesity. The purpose of this model is to determine how the altered maternal physiology may lead to an increased risk of metabolic complications in the offspring. While many organ systems have been demonstrated to be affected by maternal HFD and obesity in our NHP model, my research specifically focuses on the development of $\beta$ cells and $\alpha$ cells in these animals. Due to the counter-regulatory nature of these cells, it is necessary to examine effects in both, rather than specifically focusing on the $\beta$ cell, as many other studies have done. In the next chapter, I will characterize the model, explain the specific effects that have been demonstrated in other organ systems thus far and describe the specific aims of my studies in these offspring. 
Chapter 1 Figures

Comstock, 23 


\section{Figure 1.1: Metabolic Syndrome}

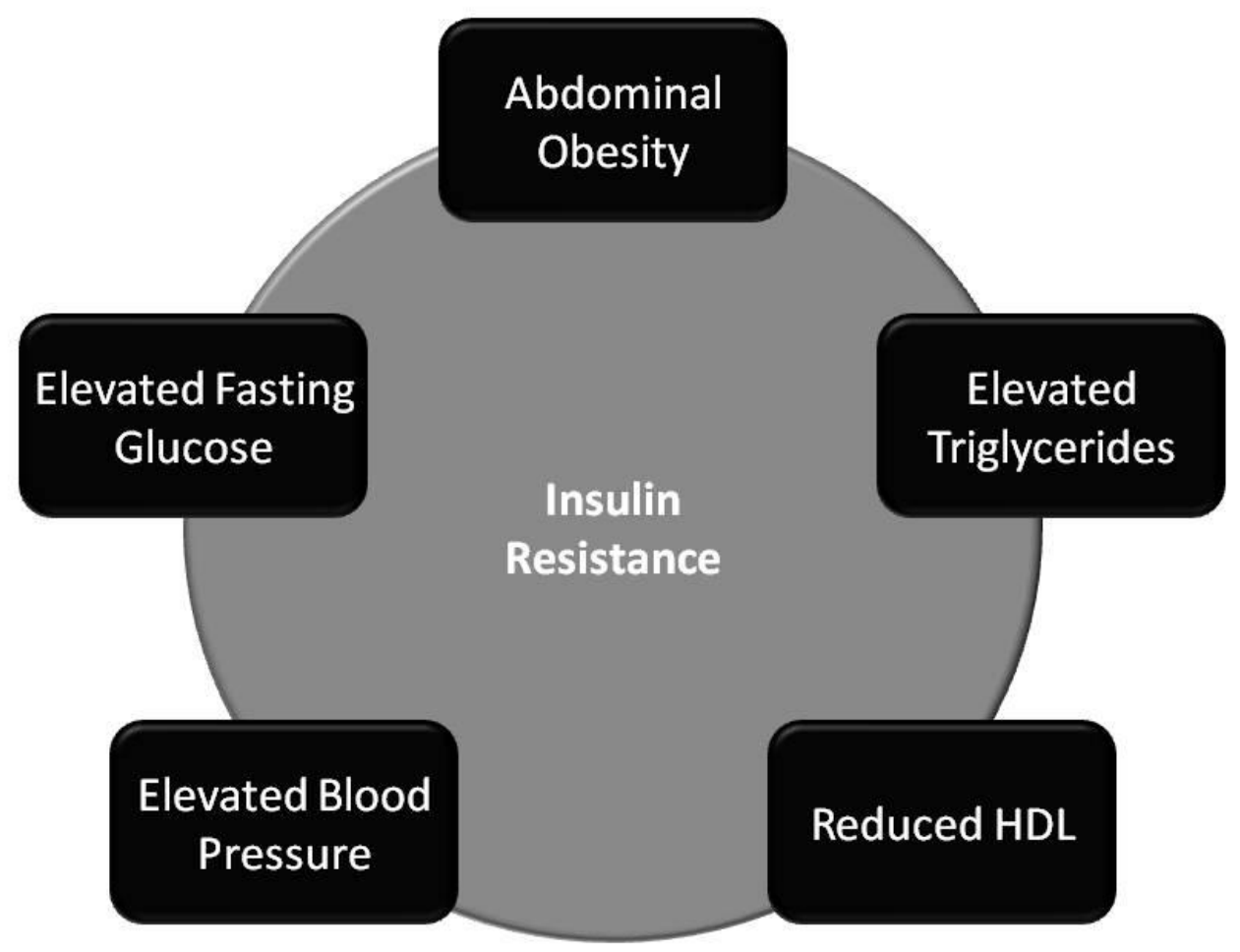

Metabolic syndrome is a clustering of health effects that increase an individual's risk for developing cardiovascular disease and T2DM. The International Diabetes Federation defines this disorder as abdominal obesity with two other associated symptoms listed above, while the American Heart Association identifies this syndrome as a clustering of any three symptoms listed above. Most organizations recognize insulin resistance as a common etiology that leads to this disease. 
Figure 1.2: Diagram of Glucose Homeostasis

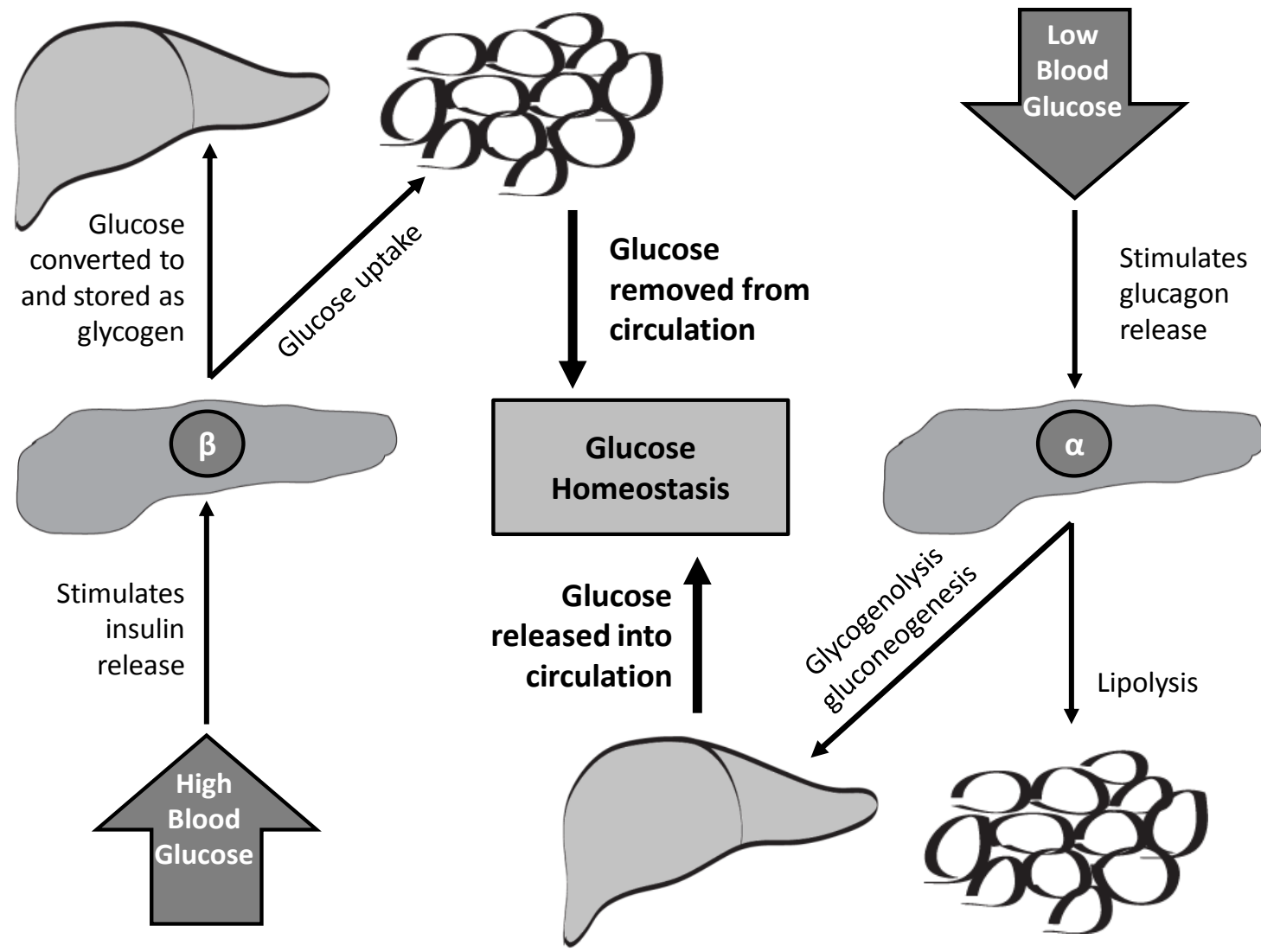

Whole-body glucose homeostasis is maintained by the hormones insulin and glucagon.

When glucose levels rise, $\beta$ cells secrete insulin. Insulin binds to its receptor on various tissues and allows for glucose uptake, utilization and storage. When glucose levels fall, glucagon is released from $\alpha$ cells. This leads to lipolysis of fat stores and causes the liver to undergo glycogenolysis and gluconeogenosis which releases glucose into circulation. 
Figure 1.3: Diagram of Fully Formed Pancreas

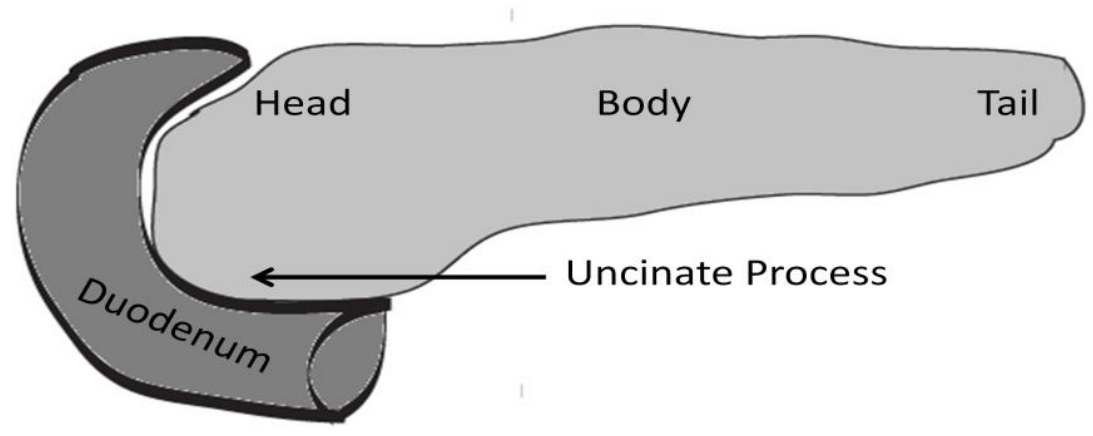

During embryonic development, the duodenal c-shape is formed due to the rotation of the ventral pancreatic bud into the dorsal bud. After the buds fuse, the dorsal pancreatic bud becomes the body and tail of the pancreas and the ventral pancreatic bud forms the head and uncinate process. 
Figure 1.4: Glucose-stimulated Insulin Secretion in the $\beta$ cell

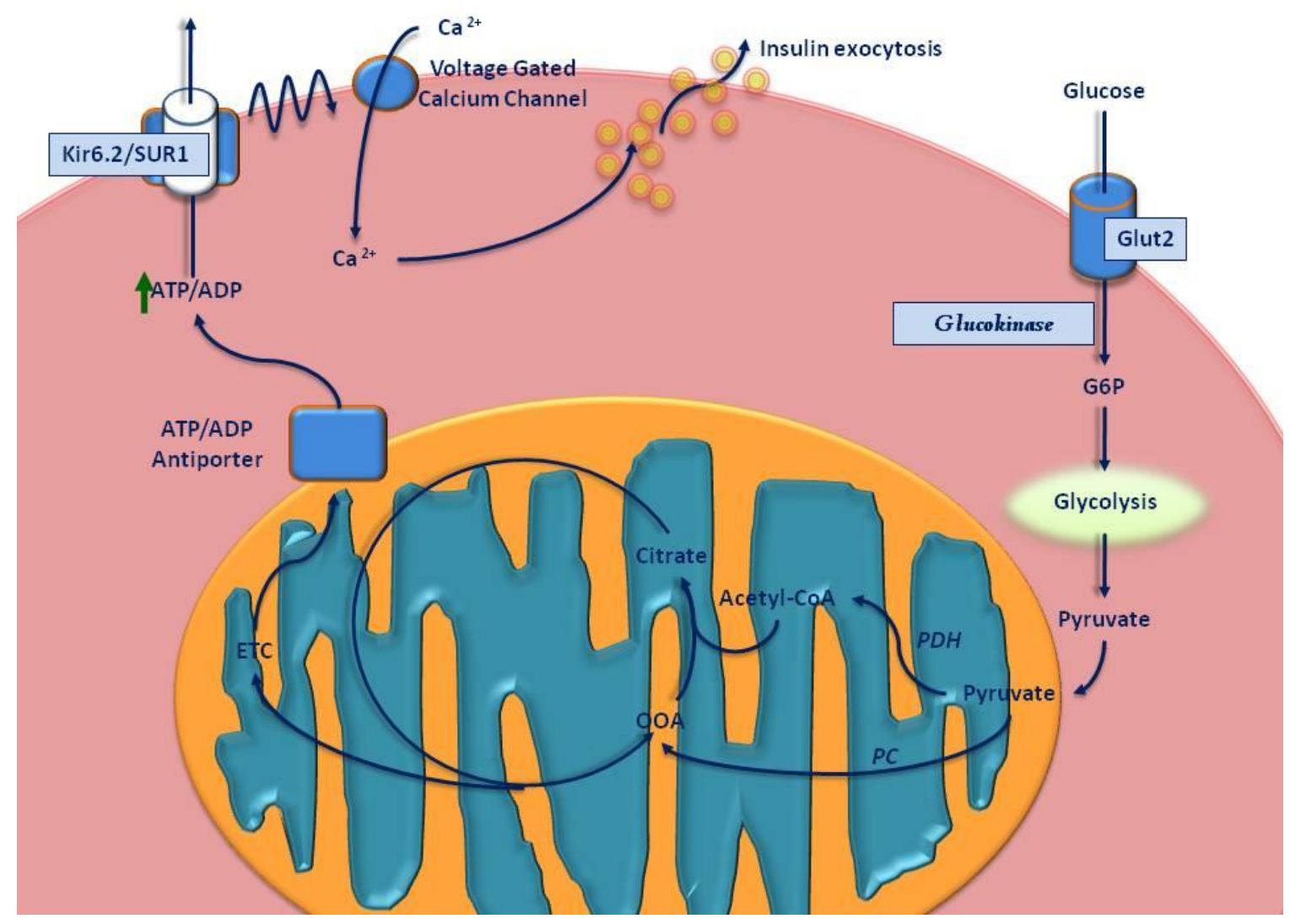

Glucose is transported into the $\beta$ cell by the facilitated glucose transporter, Glut2. It is broken down by the enzyme glucokinase (GK) into glucose-6-phosphate, which undergoes glycolysis to produce pyruvate. Pyruvate is transported into the mitochondria and metabolized to produce ATP. The rise in intracellular ATP/ADP ratio leads to a closure of ATP sensitive potassium (KATP) channels on the cell membrane, which causes depolarization of the cell and $\mathrm{Ca} 2+$ influx via voltage gated calcium channels. The influx of calcium leads to insulin granule exocytosis. 
Chapter 1 Table

Comstock, 28 
Table 1.1: Embryonic/Fetal Development of the Pancreas in Macaques and Humans

\begin{tabular}{|c|c|c|}
\hline $\begin{array}{l}\text { Macaque } \\
\text { (day) }\end{array}$ & $\begin{array}{l}\text { Human } \\
\text { (day) }\end{array}$ & Development \\
\hline 15.5 & 26 & Dorsal pancreatic bud first appears \\
\hline 16 & 26.5 & Ventral pancreatic bud appears less than a day after the dorsal bud \\
\hline 21 & 35 & $\begin{array}{l}\text { The stomach rotates to the left and the ventral bud rotates around } \\
\text { the forgut and comes in contact with the dorsal bud; the gland } \\
\text { grows as a single unit }\end{array}$ \\
\hline 32 & 52 & $\begin{array}{l}\text { Pancreas resembles the adult pancreas with a tail, body, head \& } \\
\text { uncinate process. }\end{array}$ \\
\hline 33 & 54 & $\begin{array}{l}\text { First epithelial cells stain for insulin, first beta cells begin to develop } \\
\text { and a fine capillary network is formed }\end{array}$ \\
\hline 36 & 59.5 & $\begin{array}{l}\text { Glucagon and somatostatin expressed separately in isolated } \\
\text { epithelial cells }\end{array}$ \\
\hline 38 & 63 & Endocrine cell precursors begin to form \\
\hline 38 & 63 & $\begin{array}{l}\text { Acini begin to develop and islet cell precursors begin to form and } \\
\text { clump off }\end{array}$ \\
\hline 38 & 63 & Ducts grow and branch until this point. \\
\hline 43 & 70 & The first alpha cells begin to differentiate. PP cells are now detected \\
\hline 47 & 77 & Delta cells first appear \\
\hline 51 & 84 & Rare endocrine and exocrine cells are continue to differentiate \\
\hline $51-57$ & $84-98$ & $\begin{array}{l}\text { Islets begin to co-express IAPP - indicating mature is } \beta \text { cell and } \alpha \\
\text { cells; Diffuse staining PDX1 co-localized in insulin stained islets in } \\
\text { both nucleus and cytoplasm }\end{array}$ \\
\hline 60 & 98 & $\begin{array}{l}\text { Capillary beds surround and penetrate the islets by this stage; } \\
\text { Interlobular ducts are formed and acini begin to differentiate }\end{array}$ \\
\hline 67 & 112 & $\begin{array}{l}\text { Clusters of immature islet cells become vascularized and then } \\
\text { encapuslated by connective tissue and isolated from ducts }\end{array}$ \\
\hline 71 & 119 & Insulin secretion begins \\
\hline $73-85$ & $120-140$ & $\begin{array}{l}\text { Transient glucagon secretion begins; Well formed islets are } \\
\text { identifiable although isolated endocrine cells persist between the } \\
\text { acinar cells. }\end{array}$ \\
\hline 85 & 140 & $\begin{array}{l}\text { Fetal pancreas begins to demonstrate mature glucose transporter } \\
\text { transporter levels }\end{array}$ \\
\hline $114-170$ & $187-280$ & Beta cells double each day \\
\hline $114-170$ & $187-280$ & Islet remodeling occurs \\
\hline Birth & Birth & Islets account for $4 \%$ of the total pancreas \\
\hline
\end{tabular}

Comstock, 29 
Chapter 2: Model and Study Design

Comstock, 30 


\section{A Nonhuman Primate Model of Maternal HFD Consumption}

The Grove laboratory at the Oregon National Primate Research Center (ONPRC) uses a Japanese macaque model to investigate the relationship between maternal HFD consumption, maternal obesity and health risks in the offspring. Macaques are an essential animal model to study the progression of obesity because they are phylogenentically close to humans (Isa, Yamane, Hamai, \& Inagaki, 2009). Their body structure, physiology, gestation and development have been well characterized and are more similar to humans than other commonly used laboratory animal models (i.e. rodents and sheep) (Hamada, 1999). This model has been developed and used for the past 10 years to characterize effects of HFD consumption in adult females as well as their offspring.

Adult female NHPs, ages 5 to 7, were randomly assigned to this project from a larger breeding colony that has been at ONPRC since the 1960s. Females were age- and weight-matched at the beginning of this study and placed into two separate groups. One group was fed a normal, monkey chow control (CTR) diet supplying $13 \%$ of calories from fat. The fat in the CTR diet is principally made of vegetable fat. The experimental group was fed a high-calorie, high-fat diet (HFD) supplying $35 \%$ of calories from fat plus calorically dense treats. This HFD is made from animal fats, principally lard, and is rich in total fatty acids, saturated, and monounsaturated fat. Furthermore, this diet is balanced in essential fatty acids but has an increased n- 6 to n-3 polyunsaturated fatty acid ratio that is similar to the current Western diet (Grant et al., 2011). The animals were maintained on their respective diets until they reach early middle age (12 to 14 years of age).

Comstock, 31 
All females were socially housed in indoor/outdoor housing with two males and approximately 8 to 10 females per group. They were allowed to breed naturally and become pregnant. The adult females have been extensively characterized during each year of the study throughout the pre-gravid, pregnant, and lactation periods. Specifically, animals were weighed monthly and checked for pregnancy throughout the breading season (November through May). Dual-energy X-ray absorptiometry (DEXA) scans were performed annually on each animal during the Fall (August - October). IVGTTs were performed twice each year; once prior to breeding season and a second time at approximately 123 days gestation (during the early third trimester). Like humans, the HFD dams vary in their sensitivity to the diet. They are classified as either sensitive or resistant to the diet based on their insulin clearance rates in response to an IVGTT at the end of the second year on the diet, according to methods previously described (McCurdy et al., 2009). To be classified as resistant to the diet, the HFD animal's insulin area under the curve (IAUC) must fall within 2 standard deviations of the mean of the control animals for that same year (Fig. 2.1a).

A correlation analysis between the IAUC at the end of year 2 and body weight of these animals at the end of year 4 demonstrated a significant correlation (Pearson correlation $=0.46, \mathrm{p}=0.0092$ ), indicating that insulin sensitivity at 2 years is a predictor of long-term weight gain in these animals (Fig. 2.1b). Concurrent with this finding, all HFD animals during year 4 displayed an increase in body weight and body fat (Fig. $1.1 c \& d)$. However, this increase was significantly more severe in the animals that were classified as diet sensitive (HFD-S) versus the resistant (HFD-R) animals. A similar 
response has been established in various rodent models in which a subset of animals exposed to a HFD are more resistant to obesity (Enriori, et al., 2007; Speakman, Hambly, Mitchell, \& Krol, 2007).

Some offspring from the CTR and HFD mothers were collected by C-section at gestational day 130 (G130). Since the normal pregnancy term in a Japanese macaque is approximately 173 days (Hayama, Nakiri, \& Konno, 2011), G130 corresponds to the early third trimester of development, a critical period of islet development within the fetus (Fowden \& Hill, 2001) . Likewise, in order to investigate the effects of post-natal HFD consumption, a subset of the CTR and HFD offspring were born by natural means and maintained with their mothers in the same housing and on the same diet until weaning at approximately 7 to 9 months of age. At this time, the offspring were weaned and placed into a social housing facility with peers, where they were maintained on their same diet or switched to the opposing diet for approximately 5 months. This produced four post-weaning groups: CTR/CTR, CTR/HFD, HFD/CTR and HFD/HFD (Table 2.1).

As indicated above, the purpose of this model is to investigate the effects of HFD consumption on fetal and early post-natal pancreatic development. We have chosen the HFD as a model of overfeeding that is similar to the Western diet. By providing a highly saturated fatty diet that is also calorically dense to our animals, we believe that we can mimic the detrimental effects of the Western diet and begin to understand some of the mechanisms that are leading to the health complications in children today. Also, by allowing animals to be maintained with their mothers until weaning, we are endeavoring to mimic the early lifestyle in humans. If a mother eats a healthy diet throughout her 
pregnancy, she is likely to continue to do so during the breast-feeding period. The opposite is true as well - a mother who eats fatty foods throughout her pregnancy will not be likely to change her lifestyle during breastfeeding. While the CTR/CTR and the HFD/HFD juvenile groups are best representative of the human population, the two opposing groups, CTR/HFD and HFD/CTR are essential to test which might be the factors (maternal diet versus post-weaning diet) that lead to the complications that we demonstrate in these offspring. 


\section{Metabolic Syndrome and Type 2 Diabetes Mellitus in the Macaque}

T2DM has been demonstrated to develop spontaneously in macaques as they reach middle age (approximately 15 years of age), which is preceded by typical symptoms of MetS in these animals. Macaques that develop MetS are obese, have increased waist circumference, are insulin resistant and have elevated triglycerides, lower HDL and increased fasting plasma glucose levels, when compared to healthy macaques (Hansen \& Bodkin, 1990; Wagner et al., 2001; X. Zhang et al., 2011). MetS occurs spontaneously in approximately $5-15 \%$ of both young and middle aged macaques that eat a healthy diet (Kaufman et al., 2005; X. Zhang, et al., 2011), while T2DM occurs in nearly one third of macaques with MetS-like effects (Hansen \& Bodkin, 1986).

While not all of the above mentioned markers of MetS were measured, in our macaque model, MetS-like effects occur in approximately 13\% (4/31) of CTR females and are characterized by elevations in fasting plasma insulin, fasting blood glucose, body weight and body fat (Fig. 2.2). In contrast, MetS occurs in approximately 72\% (26/36) of age matched HFD females that have consumed the diet for 4 years or more, while $28 \%$ of remain resistant to MetS-like effects. All females that have consumed the HFD for 4 years or more are at least 9 years old; however, those that develop MetS tend to be about 2 years older than the average HFD female. Of the females who developed MetS, $12 \%$ (3/26) went on to develop T2DM, which is characterized by an average fasting blood glucose level of $117 \mathrm{mg} / \mathrm{dL}$ in these animals (Fig. 2.2). T2DM has not occurred spontaneously in the CTR animals; however, females are removed from the study before the onset of middle age.

Comstock, 35 


\section{Effect of Maternal HFD Consumption on NHP Offspring}

We have previously demonstrated significant effects on fetal and early post-natal development due to exposure to the HFD or maternal obesity. Maternal HFD consumption translated to an increased n-6 to n-3 polyunsaturated fatty acid ratio in the fetal offspring, with a reduction in the essential fatty acid docosahexaenoic acid (DHA). This diet also decreased the supply of DHA to the nursing offspring through breast milk, while increasing total insulin content of the breast milk (Grant, et al., 2011). This could result in serious defects in the offspring, as DHA is essential for neuronal formation and cognitive function (Uauy et al., 1996).

Placental studies established a significant reduction in uterine volume blood flow from HFD animals and a significant increase in inflammatory cytokines regardless of maternal sensitivity to the HFD. HFD consumption by obese mothers with hyperinsulinemia also reduced volume blood flow on the fetal side of the placenta and resulted in increased number of placental infarctions and stillbirths. These studies indicate that a HFD, independent of obesity, may lead to placental insufficiency, and that maternal obesity and insulin resistance results in decreased blood volume to the fetus (Frias et al., 2011). The reduction in blood flow and the increase in cytokines are likely to have a significant effect on various developing organs.

In fact, we have demonstrated an increase in local inflammatory response within the brain of the HFD fetus. Specifically, we demonstrated disturbances in the serotonergic system, which led to increased anxiety in the female postnatal offspring 
from HFD mothers while males tend to be more aggressive in response to stress (Sullivan et al., 2010). Also, within the melanocortin system, agouti-related peptide (AGRP) mRNA expression and protein immunoreactivity were inhibited, while proopiomelanocortin (POMC) mRNA expression was upregulated in hypothalamic neurons of HFD fetal offspring (Grayson et al., 2010). These neuronal populations play a key role in glucose homeostasis by sensing both glucose and insulin (Fioramonti et al., 2007; Parton et al., 2007; Woods, Lotter, McKay, \& Porte, 1979) and integrating this signal by regulating hepatic glucose production (HGP) (Benoit et al., 2002; Pocai et al., 2005; van den Hoek et al., 2004). Since AGRP neurons mediate the insulin inhibition of hepatic glucose production (Konner et al., 2007), the inhibition of this peptide will likely lead to increased HGP.

Accordingly, we demonstrated that fetal offspring from mothers in years 2 through 4 on the HFD had increased expression of hepatic gluconeogenic genes and transcription factors. In addition, we demonstrated an increase in liver TGs and evidence of hepatic oxidative stress early and increased hepatic apoptosis, consistent with the development of nonalcoholic fatty liver disease (NAFLD) (Grant, et al., 2011; McCurdy, et al., 2009). Notably, the increased hepatic TG levels persisted until post-natal day 180 (P180), indicating that early HFD exposure through the maternal diet will likely increase the risk for early-onset NAFLD.

Finally, the HFD leads to aortic endothelial dysfunction in animals exposed to the diet until 13 months of age (Fan et al., 2012). Endothelial dysfunction has been established as an early marker for atherosclerosis, indicating that the HFD offspring are at 
increased risk for developing cardiovascular disease (Celermajer, Sorensen, Bull, Robinson, \& Deanfield, 1994; Ludmer et al., 1986; Reddy, Nair, Sheehan, \& Hodgson, 1994). Taken together, these results indicate that maternal HFD consumption places increased metabolic pressure on the developing offspring. The increased liver gluconeogenesis, elevation of circulating TGs and endothelial dysfunction indicate that the HFD offspring may be susceptible to development of pediatric MetS and may ultimately progress to T2DM. The purpose of my research is to determine how HFD consumption by the mother and the post-natal offspring affect pancreas development. It is necessary to determine if maternal HFD or obesity during pregnancy will lead to effects on the pancreas that will increase their susceptibility to MetS. Furthermore, since early life post-natal nutrition also plays a significant role in the risk for MetS and T2DM, it is essential to determine if HFD consumption during the post-natal period will contribute to this risk. It is my aim to establish how the pancreas responds to the increased metabolic pressure of maternal HFD consumption previously described in these offspring, and how this will affect long-term pancreas function. 


\section{Specific Aims}

The first aim of this study was to determine if maternal HFD and/or obesity consumption during fetal development will affect islet cell morphology or function. I hypothesized that HFD during development would similarly suppress islet development of fetal offspring regardless of maternal obesity. Since maternal HFD consumption leads to similar effects on fetal liver TG content and upregulation of gluconeogenic genes within these livers, it is likely that pancreatic islets will be similarly affected by the diet. These islets would need to compensate for the increased glucose production of the liver and elevation in FFA supplied by the maternal HFD and would, thus, display similar effects regardless of maternal sensitivity to the diet.

My second aim was to characterize the metabolic profile of juvenile Japanese macaques chronically exposed to a HFD. Specifically, I analyzed the physiology of these animals up through weaning to determine if chronic HFD exposure affected glucose homeostasis. I hypothesized that the effect on islets caused by HFD during gestation would lead to disregulation of insulin secretion and increased susceptibility of the HFD offspring to insulin resistance. While fetal exposure to the HFD should prepare the animal for continued post-natal HFD exposure, the increased liver TG deposition will likely lead to insulin resistance in this organ. This insulin resistance will likely be compensated by an increase in insulin secretion in the HFD offspring.

My third aim was to determine if the changes in islet mass would persist after the weaning period and lead to persistent changes in glucose homeostatic mechanisms. I 
hypothesized that chronic HFD exposure in the HFD/HFD juvenile would lead to hyperinsulinemia, increased insulin resistance, and persistent changes in islet morphology. By returning the HFD animals to a CTR diet after weaning (HFD/CTR) these changes would be ameliorated. Finally, it was my hypothesis that post-weaning HFD alone (CTR/HFD) would lead to alterations in islet morphology that would have a minimal affect on glucose homeostasis in these animals. I have reported the results of the experiments testing these hypotheses in the following pages and have included the methodologies that I used. 
Chapter 2 Figures

Comstock, 41 
Figure 2.1: Characterization of CTR and HFD Dams
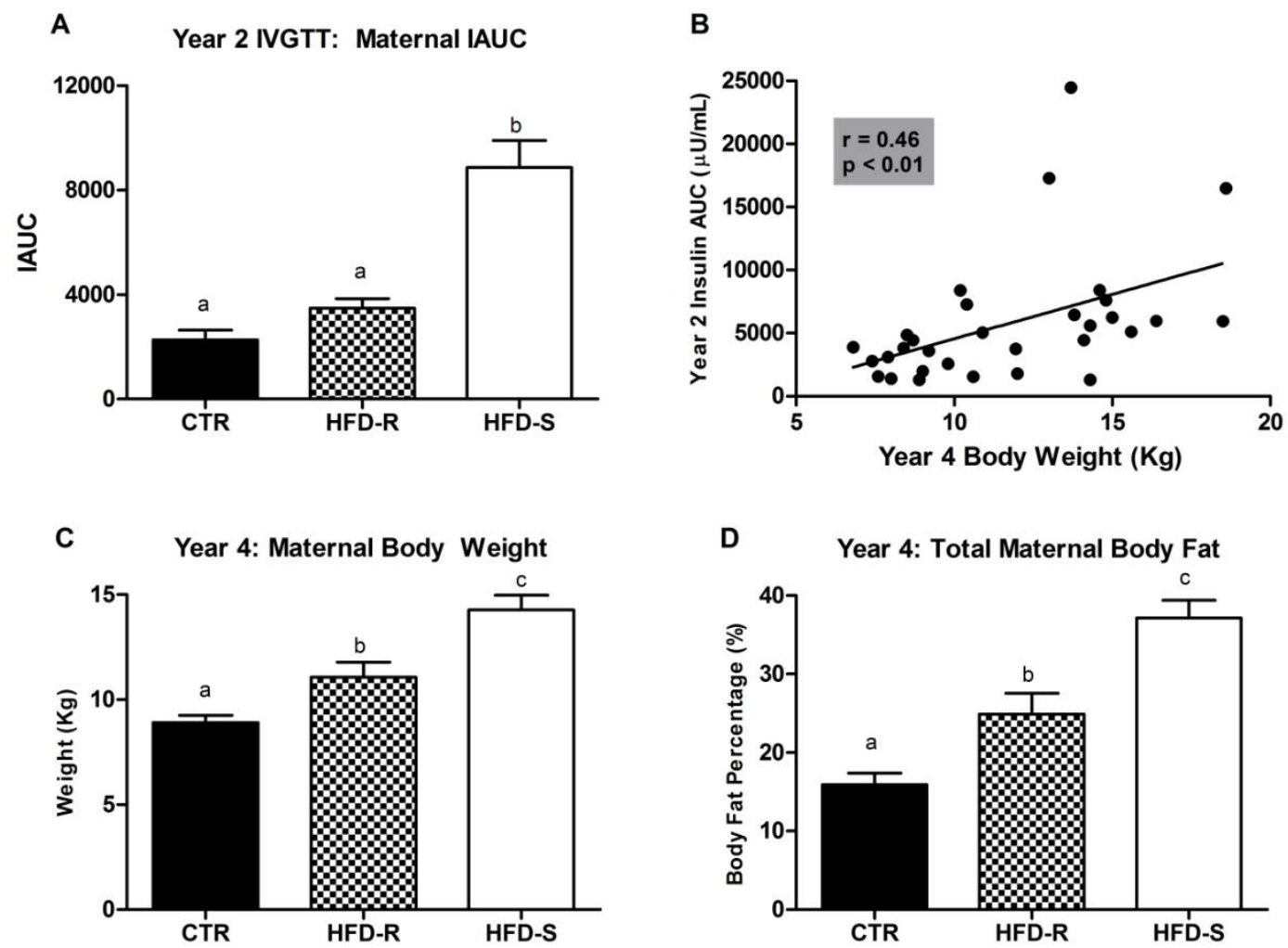

IAUC after 2 years in the study is significantly increased in HFD-S but not HFD-R dams (A). This correlates with body weight in dams after 4 years on HFD (B). Weight (C) and body fat percentage (D) of dams after 4 years is significantly increased due to HFD and HFD-S display a further increased over the HFD-R group. 
Figure 2.2: Characterization of Metabolic Syndrome and Diabetes in Females

A

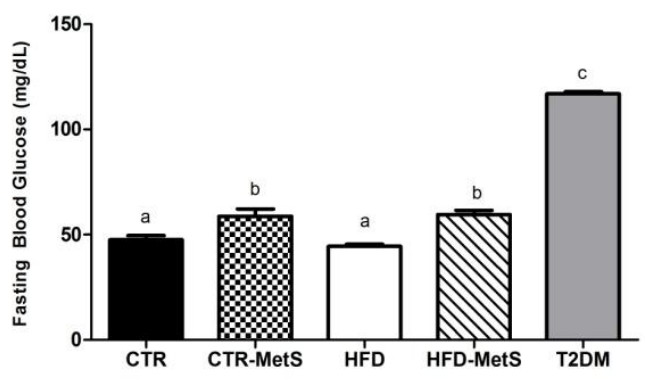

C

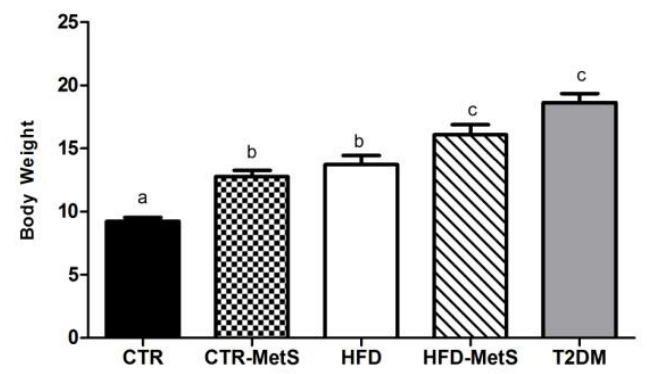

B

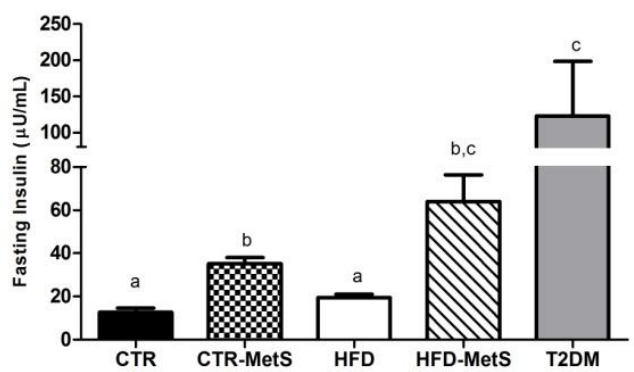

D

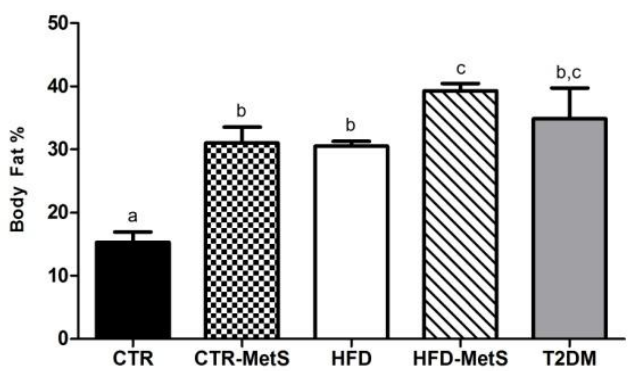

Females that have MetS have elevated fasting blood glucose (A), elevated fasting insulin (B), increased body weight (C) and increased body fat (D) compared to others on the same diet. Females that develop T2DM have increased fasting blood glucose, increased fasting insulin and elevated body weight when compared to all other groups (A-C). Body fat percentage was not significantly different from HFD female or other groups that developed MetS (D) 
Chapter 2 Table

Comstock, 44 
Table 2.1: Post-natal offspring groups

\begin{tabular}{|c|c|c|c|}
\hline Group & Pre-natal & Pre-weaning & Post-weaning \\
\hline CTR/CTR & & CTR & CTR \\
\hline CTR/HFD & & CTR & HFD \\
\hline HFD/CTR & & HFD & CTR \\
\hline HFD/HFD & & HFD & HFD \\
\hline
\end{tabular}


Chapter 3: Maternal High-fat Diet Consumption During Pregnancy Leads to

Reduced $\alpha$ Cell Mass in Fetal Offspring

Comstock, 46 


\section{Introduction}

The mechanisms that lead to the association between maternal obesity, childhood obesity and metabolic syndrome need to be determined in order to treat or prevent this effect from perpetuating. We have previously demonstrated that HFD consumption during pregnancy, independent of maternal metabolic phenotype, can lead to a broad range of complications in fetal development that may increase these animals' susceptibility to obesity and diabetes later in life. We have demonstrated increased liver TGs, a shift in genes involved in hepatic gluconeogenesis, and increased apoptosis, suggesting early signs of NAFLD (Grant, et al., 2011; McCurdy, et al., 2009). We also observed placental insufficiency and inflammation in pregnant macaques fed a HFD. Importantly, the cytokines produced in the placenta are secreted specifically into the developing fetus, where we hypothesized that they initiate inflammatory responses in several developing organs (Frias, et al., 2011). Also, because placental insufficiency has been demonstrated to lead to reduced islet vascularization, it is important to determine how this will effect fetal islet cell development and function (Fahy, 2009; Martin-Gronert \& Ozanne, 2007).

The purpose of this study was to determine the effects of maternal obesity and/or HFD consumption during pregnancy on the development of fetal pancreatic islets. Principally, I chose to examine islet morphology, $\alpha$ cell and $\beta$ cell mass, and regulation of genes involved in glucose metabolism and islet development. Previous animal model studies indicate that much of the disregulation of glucose homeostasis in offspring associated with in-utero obesity exposure is due to $\beta$ cell dysfunction or the suppression 
of $\beta$ cell proliferation in response to this early life insult (Bertin et al., 2002; Hales et al., 1991; Van Assche, De Prins, Aerts, \& Verjans, 1977; L. Zhang, et al., 2011). However, recent data has demonstrated the complexity of pancreatic islet paracrine regulation of both $\beta$ cells and $\alpha$ cells that are disrupted in the development of T2DM (Ehses, Ellingsgaard, Boni-Schnetzler, \& Donath, 2009; Ellingsgaard et al., 2011). It is important to understand how $\alpha$ cells may also be affected by maternal HFD consumption. I chose to investigate these hypotheses in our well-characterized model that has developmental similarities to humans and in which we can control and manipulate their environment. My aim was to determine which islet cells may be affected by maternal obesity and/or HFD consumption. I hypothesized that HFD during development would similarly suppress islet development of fetal offspring regardless of maternal obesity. Since maternal HFD consumption leads to effects on fetal liver triglyceride content and upregulation of gluconeogenic genes within these livers, it is likely that pancreatic islets will be similarly affected by the diet. These islets would need to compensate for the increased glucose production of the liver and elevation in FFA supplied by the maternal HFD and would thus display similar effects regardless of maternal sensitivity to the diet. 


\section{Materials and Methods}

Animals. All animal procedures were in accordance with the guidelines of the Institutional Animal Care and Use Committee of the ONPRC as previously described (Frias et al.; Grant et al.; Grayson et al.; McCurdy, et al., 2009; Sullivan et al.). Briefly, age and weight matched adult female Japanese macaques were fed a control (CTR) or HFD for up to 4 years. Mothers were fasted overnight and fetal offspring were obtained by c-section G130 (early $3^{\text {rd }}$ trimester) and immediately delivered to necropsy for tissue collection. HFD fetal offspring used for this study were taken from mothers in their $4^{\text {th }}$ year on the HFD (Table 3.1).

Intravenous Glucose Tolerance Tests. In the dams we performed the IVGTTs at 123 days ( \pm 5 days) gestation. Mothers were fasted overnight prior to a morning IVGTT, while offspring were fasted for 5 hours and the IVGTT was performed in the afternoon. Animals were sedated with ketamine $(10 \mathrm{mg} / \mathrm{kg})$ and administered a glucose bolus $(50 \%$ dextrose solution) at a dose of $0.6 \mathrm{~g} / \mathrm{kg}$ via the saphenous vein. Baseline blood samples were obtained prior to the infusion, and $500-\mu \mathrm{L}$ blood samples were taken at $1,3,5,10$, 20, 40, and 60 min after infusion via the femoral artery. Glucose was measured immediately using a OneTouch Ultra2 Blood Glucose Monitor (LifeScan), and the

remainder of the blood was kept in heparinized or clot tubes on ice for insulin measurement. After the IVGTT, samples were centrifuged, and plasma was stored with $5 \%$ aprotinin at $-80^{\circ} \mathrm{C}$ until assayed. Insulin was assayed in plasma by RIA (Millipore catalog no. RI-13K, Billerica, MA). HOMA-IR was calculated as fasting serum insulin $(\mu \mathrm{U} / \mathrm{ml}) \times$ fasting plasma glucose $(\mathrm{mmol} / \mathrm{l}) / 22.5$ as previously described (Bonora et al., 
2000; Matthews et al., 1985). Glucose-stimulated insulin secretion was measured as the insulin area under the curve (IAUC) minus basal fasting insulin levels, while total IAUC is a measure of insulin secretion from time 0. Baseline samples were assayed for glucagon (Millipore, catalog no. GL-32K, Billerica, MA), c-peptide (Roche Diagnostics, catalog no. 03184897190, Indianapolis, IN) and leptin (Millipore catalog no. HL-81K, Billerica, MA).

.RNA Isolation and Quantitative RT-PCR. Total RNA was isolated from 5 head-to-tail grouped sections of each offspring pancreas (Fig. 3.1) using TRIzol reagent (Invitrogen, Carlsbad, CA). RNA was bioanalyzed with an Agilent Bioanalyzer (Scotland, UK) and only high quality RNA was DNase treated and reverse-transcribed. mRNA was measured by real-time quantitative RT-PCR using a PE Applied Biosystems PRISM 7700 Sequences Detector System. Premade qPCR gene expression assays directed against human sequences were purchased from Applied Biosystems. The amplification was performed as follows: $2 \mathrm{~min}$ at $50^{\circ} \mathrm{C}, 10 \mathrm{~min}$ at $95^{\circ} \mathrm{C}$, then 45 cycles each at $95^{\circ} \mathrm{C}$ for $15 \mathrm{sec}$ and at $60^{\circ} \mathrm{C}$ for $60 \mathrm{sec}$ in the $\mathrm{ABI} /$ Prism 7700 Sequences Detector System. Standard curves on serial dilutions of the cDNA (1:10, 1:50, 1:100 1:500, 1:1000 and 1:10,000) were done using cDNA from pooled pancreas samples. Standard curves were drawn on the basis of the log of the input RNA versus the critical threshold (CT) cycle, in which the fluorescence of the sample is greater than the threshold of the baseline fluorescence. Although expression of classical housekeeping genes 18S, $\beta$-actin, and GAPDH are commonly used to normalize gene expression levels in qRT-PCR assays, all three genes were significantly differentially expressed in the fetal groups. A novel 
reference gene, ALG9 (Kidd et al., 2007), was assayed and also shown to be differentially expressed between fetal groups. Therefore, all qPCR results are normalized to cDNA concentrations. We have also presented data as gene expression normalized to $\beta$ cell area, $\alpha$ cell area, or islet area, depending on the cell types in which the gene of interest is expressed. This methodology has been previously described when whole pancreas mRNA is used (Gatford, et al., 2008).

Immunohistochemistry. Pancreas tissue was collected from 5 head-to-tail areas (Fig. 3.1), section number 3 was fixed with $10 \%$ zinc formalin, paraffin-embedded, and cut into $5 \mu \mathrm{m}$ sections. Sections were deparaffinized in xylene and then $100 \%, 95 \%, 85 \%$, and $70 \%$ graded ethanol washes for 5 minutes each. Standard antigen retrieval with microwave-heated citrate buffer was used. Standard immunohistochemical methods were used. Briefly, sections were washed in KPBS and then blocked in $2 \%$ donkey serum in 0.4\% triton X/KPBS. Antibodies against insulin (\#AB9210, 1:2,000, Chemicon, Temecula, CA) and glucagon (\#4031-01F, 1:1500, Millipore, St. Charles, MO) were used. Primary antibodies were diluted in $2 \%$ donkey serum in $0.4 \%$ triton X/KPBS and applied to tissue sections where they were incubated overnight. Tissues were then washed in KPBS and secondary antibodies were applied for 1 hour at room temperature at a dilution of 1:200 (Jackson ImmunoResearch Laboratories, Inc, West Grove, PA).

Quantitative Analysis. Images were acquired with a MarianasTM imaging workstation (Intelligent Imaging Innovations, Denver, CO) using a 20X objective and a stereology module to montage images covering whole sections. Areas occupied by $\beta$ and $\alpha$ cells were measured based on intensity segmentation of each fluorescence channel 
using Slidebook 5.0. The $\beta$ and $\alpha$ cells fractions were calculated by dividing by the area of the whole section. Total islet area, islet mass [(total islet area/pancreas area)x pancreas weight], and $\beta$ and $\alpha$ cell mass [(total cell area/pancreas area)x pancreas weight] were calculated as previously reported (Uchida et al., 2005). 


\section{Results}

Alpha Cell Mass is Decreased in HFD Fetal Offspring. Overall, the physiological phenotype in the HFD fetus was minimally affected by the maternal phenotype or diet. HFD fetal weight was slightly reduced (Table 3.1), which is consistent with previous published findings from our lab, although it was not significant, likely due to the smaller sample size used for this study (McCurdy, et al., 2009). Furthermore, maternal HFD consumption did not lead to significant differences in pancreas weight, glucose, or insulin in the fetus (Table 3.1). However, C-peptide was significantly decreased in HFD fetuses and glucagon was decreased in fetuses from HFDsensitive (HFD-S) mothers (Table 3.1). Analysis of islets demonstrated no significant difference in total islet mass between the fetal groups (Fig. 2.2a). However, a closer analysis of specific cell types within the islets demonstrated a significant decrease in $\alpha$ cell mass in the HFD offspring (Fig. 3.3a). While glucagon mRNA expression was also significantly decreased in the HFD animals (Fig. 3.3b), when this expression was normalized to $\alpha$ cell mass, the difference was lost (Fig. 3.3c). On the contrary, there was no significant difference in $\beta$ cell mass (Fig. 3.3d) or plasma insulin between groups (Table 3.1). However, insulin gene expression was significantly decreased in the HFD offspring (Fig. 3.3e) and normalizing to $\beta$ cell area did not change this effect (Fig. 3.3f). The effect on islet cell mass caused a significant increase in the $\beta$ cell to $\alpha$ cell ratio in HFD offspring (Fig. 3.4).

Since maternally derived glucose may exert an effect islet cell development, we measured umbilical vein glucose, which is a measure of glucose delivered to the fetus 
from the placenta (Kiserud, 2005; Konig, Vest, \& Stahl, 1978), Umbilical-vein glucose significantly correlated with the $\beta$ cell to $\alpha$ cell ratio in these fetuses (Fig. 3.4b) In addition, while circulating fetal insulin was not significantly affected in the fetal HFD group, this may be due reduced clearance by the liver since c-peptide measures indicate that there is a decrease in insulin secretion. Since we have previously demonstrated an increase in gluconeogenic gene expression in the fetal HFD liver (McCurdy, et al., 2009), we chose to investigate the relationship between islet cell mass and the expression of these genes. Both $\beta$ cell mass and the $\beta$ cell to $\alpha$ cell ratio significantly correlated with expression of phosphoenolpyruvate carboxykinase 1 (PCKl) and peroxisome proliferator-activated receptor gamma, coactivator 1 alpha (PGC1A) (Fig. 3.4c-f).

Transcription Factor Expression in Fetal Pancreas. PDXI mRNA, a critical transcriptional regulator of early pancreatic development, islet cell neogenesis, and insulin gene transcription, was downregulated in the HFD fetal pancreas (Fig. 3.5a). PDX1 has also been shown to have multiple roles in pancreas development including bud expansion (Jonsson, Carlsson, Edlund, \& Edlund, 1994; Offield et al., 1996) and islet cell differentiation (Ahlgren, Jonsson, Jonsson, Simu, \& Edlund, 1998). We also examined other genes responsible for differentiation of pancreas endocrine cells. Neurogenin differentiation factor 1 (NeuroD), also known as choline dehydrogenase $\beta 2$ (BETA2), is responsible for endocrine cell differentiation and transcriptional activation of insulin secretion (Sharma et al., 1999), while NK6 homeobox 1 (NKX6.1) is more specific to expansion and maintenance of $\beta$ cells at this stage of development (Johansson \& GrapinBotton, 2002; Rivas-Carrillo, Okitsu, Tanaka, \& Kobayashi, 2007). Consistent with the 
decrease in PDX1, both NeuroD and NKX6.1 expression was decreased in the HFD fetal pancreas (Fig. 3.5b\&c).

\section{Alteration of Genes Involved in Glucose-stimulated Insulin Secretion in the}

Fetal Offspring. Since we were unable to directly test glucose-stimulated insulin secretion, we analyzed gene expression of proteins involved in this pathway, in order to determine if GSIS may be affected by the $\beta$ cell to $\alpha$ cell ratio. The two initial factors in the glucose-stimulated insulin secretion cascade are the facilitated glucose transporter 2 (Glut2) that allows glucose to enter the cell, expressed in $\beta$ cells in macaques, and glucokinase (GK), the rate-limiting enzyme in glycolysis (Iynedjian, 1993; Kramer, Moeller, Hachey, Mansfield, \& Wachtman, 2009; Matschinsky, 1996; Newgard \& McGarry, 1995). Glut2 gene expression, when normalized to $\beta$ cell area, was increased in the HFD animals (Fig. 3.6a). Since, $G K$ is expressed in both $\alpha$ cells and $\beta$ cells (Heimberg et al., 1996), we normalized $G K$ expression to islet area. $G K$ was significantly up-regulated in the HFD offspring (Fig. 3.6b). The ATP-sensitive potassium channel found in both $\alpha$ and $\beta$ cells is critical for regulating insulin and glucagon secretion. Gene expression of both components of this channel, SUR1 and Kir6.2 (Moritz, Leech, Ferrer, \& Habener, 2001), when normalized to islet area, were not significantly affected by the HFD. (Fig 3.6c\&d). Hepatocyte nuclear factor-4 alpha (HNF-4 $\alpha$ ), a transcriptional activator of KCNJ11, is required for maintenance of normal Kir6.2 mRNA levels (Gupta et al., 2005). HNF-4 $\alpha$ was also not significantly affected, when expression was normalized to islet area (Table 3.2). Further analysis of qRT-PCR results demonstrated 
that mRNA expression patterns from the fetal groups were not depended on maternal sensitivity to the HFD. 


\section{Discussion}

In the past couple of decades, there has been a clear increase in the occurrence of metabolic diseases in children (Cook, Weitzman, Auinger, Nguyen, \& Dietz, 2003; Weiss et al., 2004) and a significant contributor to this increase has been nutritional and health issues during pregnancy, commonly referred to the developmental origin of adult diseases hypothesis. However, somewhat confusingly, the increase in early-onset obesity has been shown to be associated with conditions of both undernutrition as well as overnutrition during pregnancy. This has led us to search for a common underlying cause between these opposing ends of the spectrum. Placental insufficiency is another condition that greatly increases the risk of early-onset obesity and diabetes, subsequent to fetal growth restriction and catch-up growth. Interestingly, our group recently demonstrated that chronic consumption of a HFD during pregnancy in the NHP leads to a significant reduction in placental blood flow (Frias, et al., 2011), similar to that observed in models of placental insufficiency, thus providing a possible common link between conditions of maternal undernutrition and placental insufficiency with that of maternal obesity and overnutrition.

Previously, our group has also demonstrated a premature activation of the fetal hepatic gluconeogenic pathway caused by chronic consumption of a HFD during pregnancy (McCurdy, et al., 2009). At that time it was uncertain as to what the driving factor for this activation was, especially since HFD fetal circulating glucose levels were normal. Importantly, there are numerous reports that placental insufficiency and maternal undernutrition is also associated with premature activation of the fetal 
gluconeogenesis, including an increase in fetal glucagon levels and activation of hepatic glucose production (HGP) (Fowden, Mijovic, \& Silver, 1993; Limesand, Rozance, Smith, \& Hay, 2007). This is thought to be a compensatory response to adapt to the low levels of glucose from maternal sources. This raised the question as to whether the placental insufficiency caused by the maternal HFD resulted in an increase in glucagon secretion and possibly an increase in $\alpha$ cell mass in the HFD fetal offspring. Surprisingly, in contrast to placental insufficiency and maternal undernutrition, in our current study we demonstrated a significant decrease. The correlation between hepatic gluconeogenic gene expression and both the $\beta$ cell to $\alpha$ cell ratio and $\beta$ cell mass suggests that islet cells may be adapting to normalize liver glucose secretion. This adjustment may allow the fetuses to maintain glucose homeostasis. However, while the decline in $\alpha$ cell mass may protect the fetus, this may be a maladaptive response that will ultimately lead to significant physiological consequences that are not measureable at the fetal stage. Analysis of the impact of the maternal metabolic phenotype (not shown) established that the lower $\alpha$ cell mass occurred in HFD offspring regardless of maternal obesity or insulin resistance.

Interestingly, although the decrease in $\alpha$ cell mass in HFD offspring suggests some fundamental issue with islet development, glucagon gene expression, normalized for $\alpha$ cell mass, was not significantly affected by the maternal HFD. However, the decreased glucagon secretion in the HFD-sensitive fetal offspring indicates that maternal obesity may be leading to a further decline in $\alpha$ cell function.

Comstock, 58 
A second potential contributing factor to the decreased $\alpha$ cell mass is placental glucose levels, since this was significantly correlated to $\beta / \alpha$ cell ratio (Fig. 3.4b). It is possible that the higher levels of glucose coming from the placenta are a signal that suppresses the $\alpha$ cell expansion that normally occurs during this time. Thus, while maternal HFD may have some common abnormalities in placental function to maternal undernutrition and placental insufficiency models, this does not result in a decrease in glucose delivery to through the placenta. In fact, previous reports have demonstrated an increase in glucose transporter expression in the placenta of obese mothers or mothers who consume a HFD (H. N. Jones et al., 2009; Zhu, Ma, Long, Du, \& Ford, 2010). This may result in increased glucose transfer across the placenta of HFD dams, which would impact fetal $\alpha$ cell development.

In addition, while HFD offspring have normal levels of $\beta$ cell mass, we did observe significant decreases in insulin mRNA expression. Even after normalization to the $\beta$ cell area, insulin mRNA fell to nearly $1 / 3$ of the normal expression, indicating that this is truly a decrease in expression on a per cell basis. Analysis of c-peptide from HFD offspring indicates that the decreased insulin gene transcription does translate into decreased insulin secretion, although total circulating insulin remained unchanged. In order to understand what factors may be leading to the differences in insulin expression and $\alpha$ cell mass we analyzed PDX1, NeuroD and NKX6.1. Similar to the decrease in insulin mRNA, $P D X 1$ gene transcription was also decreased. $P D X 1$ has been shown to regulate insulin gene transcription and islet expansion (Brissova et al., 2002; Jonsson, et al., 1994). NeuroD and NKX6.1 mRNA expression were also significantly decreased. 
While the specific targets of these transcription factors in this NHP fetal tissue require further investigation, it is likely that the decrease effects both insulin gene transcription and $\alpha$ cell expansion. Previous studies have demonstrated that $\alpha$ cell secretory products lead to increased PDX1 activation of insulin gene transcription (Buteau, Roduit, Susini, \& Prentki, 1999; Perfetti, Zhou, Doyle, \& Egan, 2000; Wang et al., 1999). While further studies in isolated islet are necessary to elucidate the mechanisms that lead to this disregulation the decrease in both PDX1 and insulin expression may be due to decreased paracrine regulation of $\beta$ cells caused by the decrease in $\alpha$ cell mass.

In contrast, while there have been several studies demonstrating changes in $\beta$ cell development and function in response to maternal overnutrition, undernutrition and obesity in rodent and sheep models, they have either not investigated abnormalities in the $\alpha$ cell or have reported not changes in this cell type. In ewes, maternal obesity results in accelerations in $\beta$ cell, but not $\alpha$ cell, development in the fetus in mid gestation and decreases $\beta$ cell mass in late gestation (S. P. Ford, et al., 2009; L. Zhang, et al., 2011). In rodent models, the timing of HFD consumption - beginning, middle, end or all of gestation - results in differential islet effects in fetuses of pregnant rats (Cerf, et al., 2005). Within these studies, it is not possible to determine if these differential effects are due to species differences or whether they are a result of different types of diets and/or different exposure periods to the diets. However, the effect on $\alpha$ cell mass in the NHP offspring may represent a novel, unexplored avenue that clarifies the islet defects that may occur prior to childhood obesity onset. Furthermore, because of the essential nature of islet paracrine regulation for normal endocrine function, the change in $\beta$ cell to $\alpha$ cell 
ratio may lead to islet cell dysfunction (Franklin, et al., 2005; Leung, et al., 2006). In fact, analysis of gene expression in these animals demonstrated an increase in Glut2 and GK mRNA expression in the HFD fetus, indicating that maternal HFD consumption could lead to increased glycolysis. However, since insulin gene transcription is decreased, this will decrease the reserved supply of insulin in $\beta$ cell, thereby limiting the availability of the ready releasable insulin pool and decreasing insulin secretion which we demonstrated by a decrease in c-peptide in these animals. In disassociated islets, $\beta$ cells exhibit a poor insulin secretory response, despite normal glucose metabolism (Holz, Kuhtreiber, \& Habener, 1993; Huypens, Ling, Pipeleers, \& Schuit, 2000), indicating that a basal level of glucagon or other $\alpha$ cell secretagogues that are required for efficient insulin secretion. The decreased $\alpha$ cell mass demonstrated in these animals elucidate a possible mechanism by which decreased $\beta$ cells of insulin secretion results in a disconnect between increased glucose metabolism by Glut2 and GK and decreased insulin secretion. Studies from isolated fetal islets are essential to determine how the glucose-stimulated insulin secretion mechanism may be biochemically altered. Although the decrease in $\alpha$ cell mass was unexpected due to results from other animal model studies, my hypothesis that HFD during development would similarly suppress islet development of fetal offspring regardless of maternal obesity is supported by these experiments.

Comstock, 61 
Chapter 3 Figures

Comstock, 62 


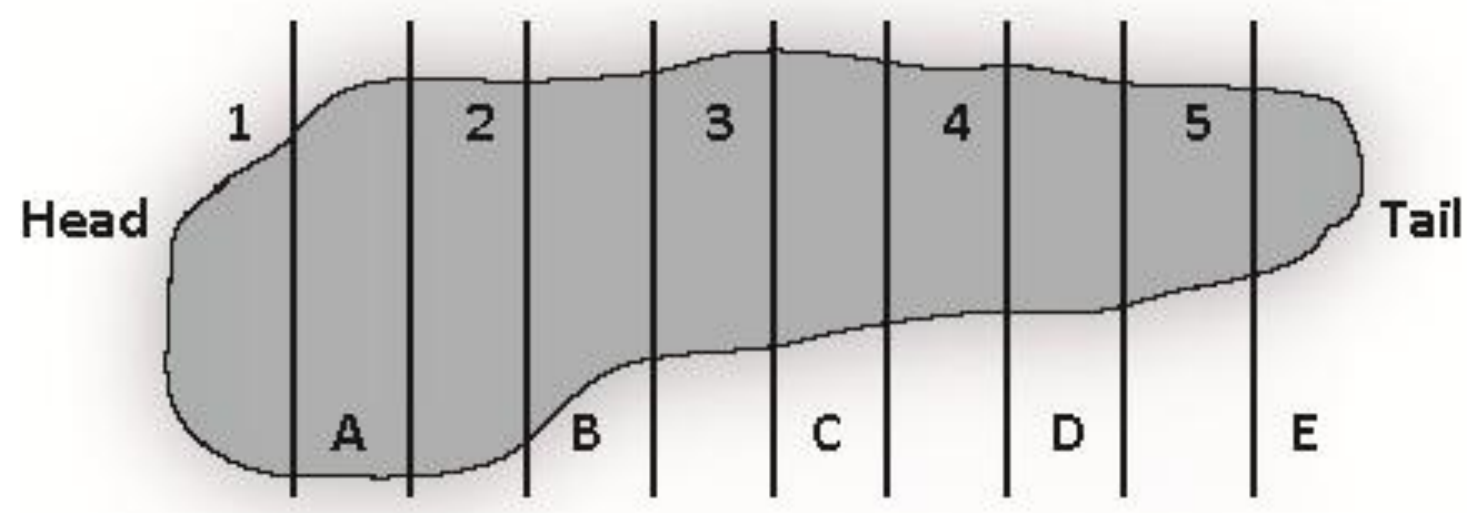

Pancreas tissue was sectioned into 10 equal head-to-tail areas. Every other section was collected for immunohistochemistry (1-5) or RNA extraction (A-B). Results from IHC were averaged together to represent the total expression in the pancreas. Fresh tissue was homogenized completely to represent the total gene expression in the pancreas. 
Figure 3.2: Immunohistochemical Analysis of Fetal Islet Mass
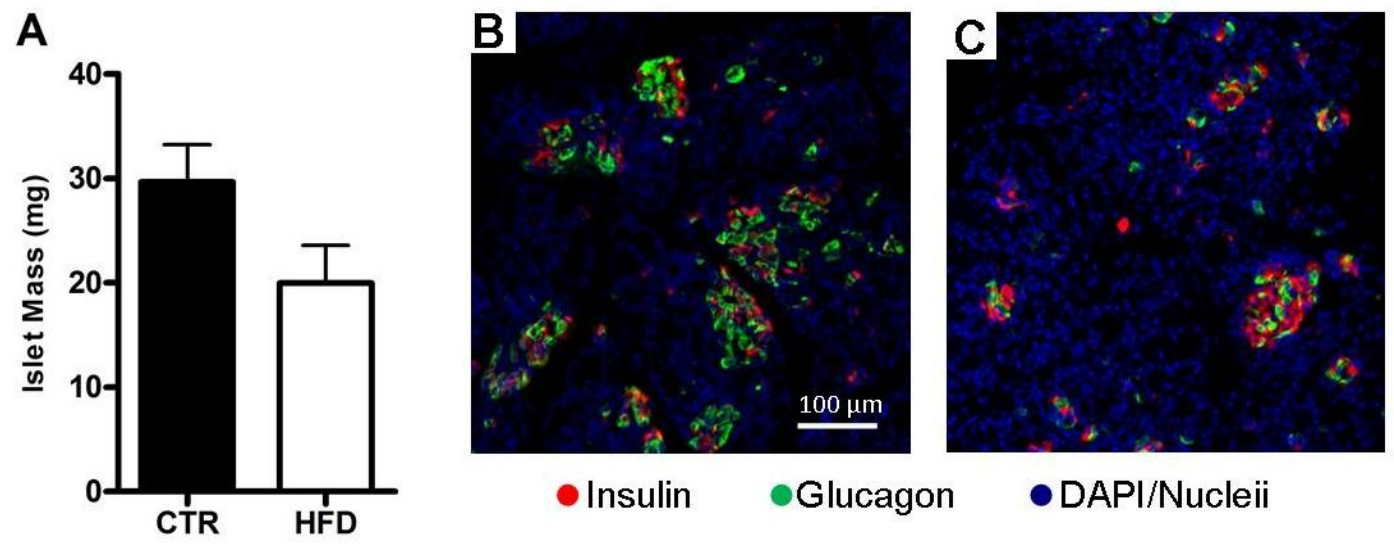

Islet mass was not significantly different between diet groups (A) Representative images of CTR (B) and HFD (C) IHC. *P $<0.05$ versus CTR (Student's t-test). 
Figure 3.3: Islet Characterization in Fetal Offspring

A
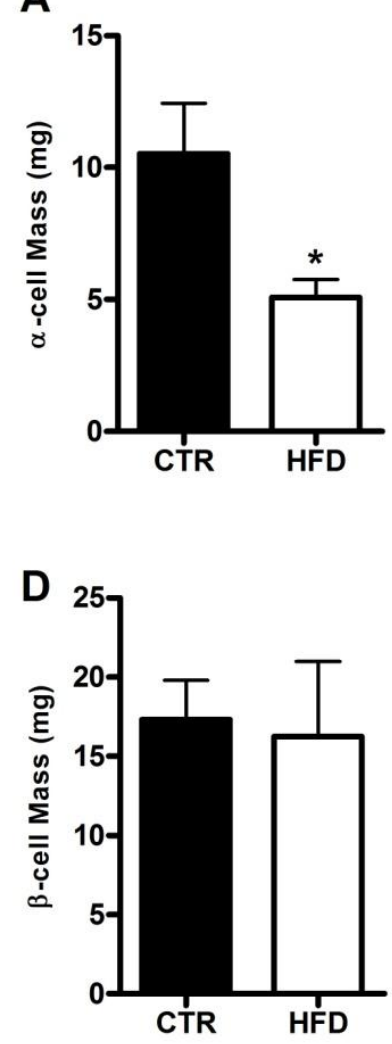

B
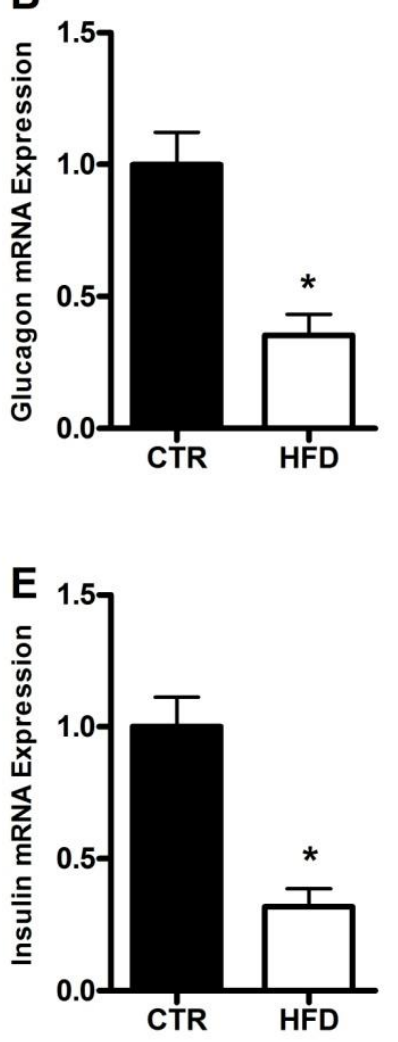

C
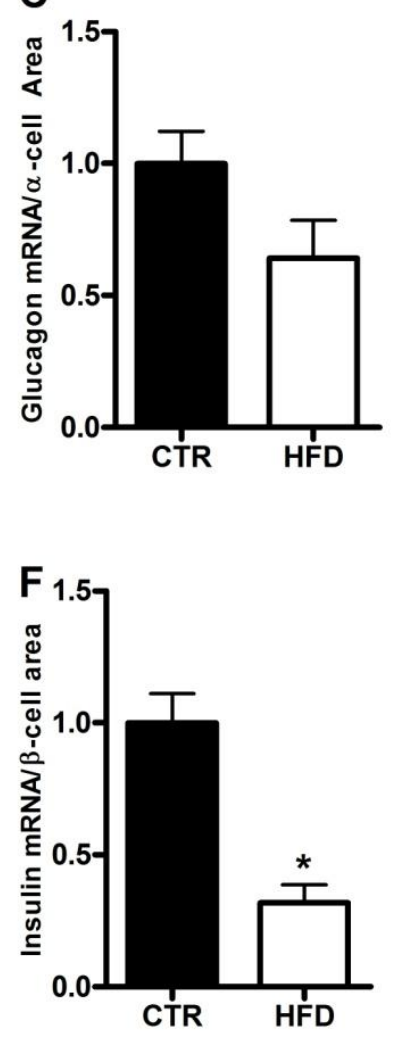

$\alpha$ cell mass was significantly decreased in the HFD fetal pancreas (A). Glucagon expression was suppressed in HFD offspring (B). When normalized to $\alpha$ cell area this effect was no longer significant (C). $\beta$ cell mass was not affected in the HFD animals (D). Insulin expression was suppressed (E) and remain suppressed when normalized to $\beta$ cell area $(\mathbf{F}) .{ }^{*} \mathrm{P}<0.05$ versus CTR (Student's t-test). IHC: CTR $\mathrm{n}=7$, HFD $\mathrm{n}=8$; Realtime: CTR $n=12$, HFD $n=8$ 
Figure 3.4: $\beta$ cell Correlation Analysis
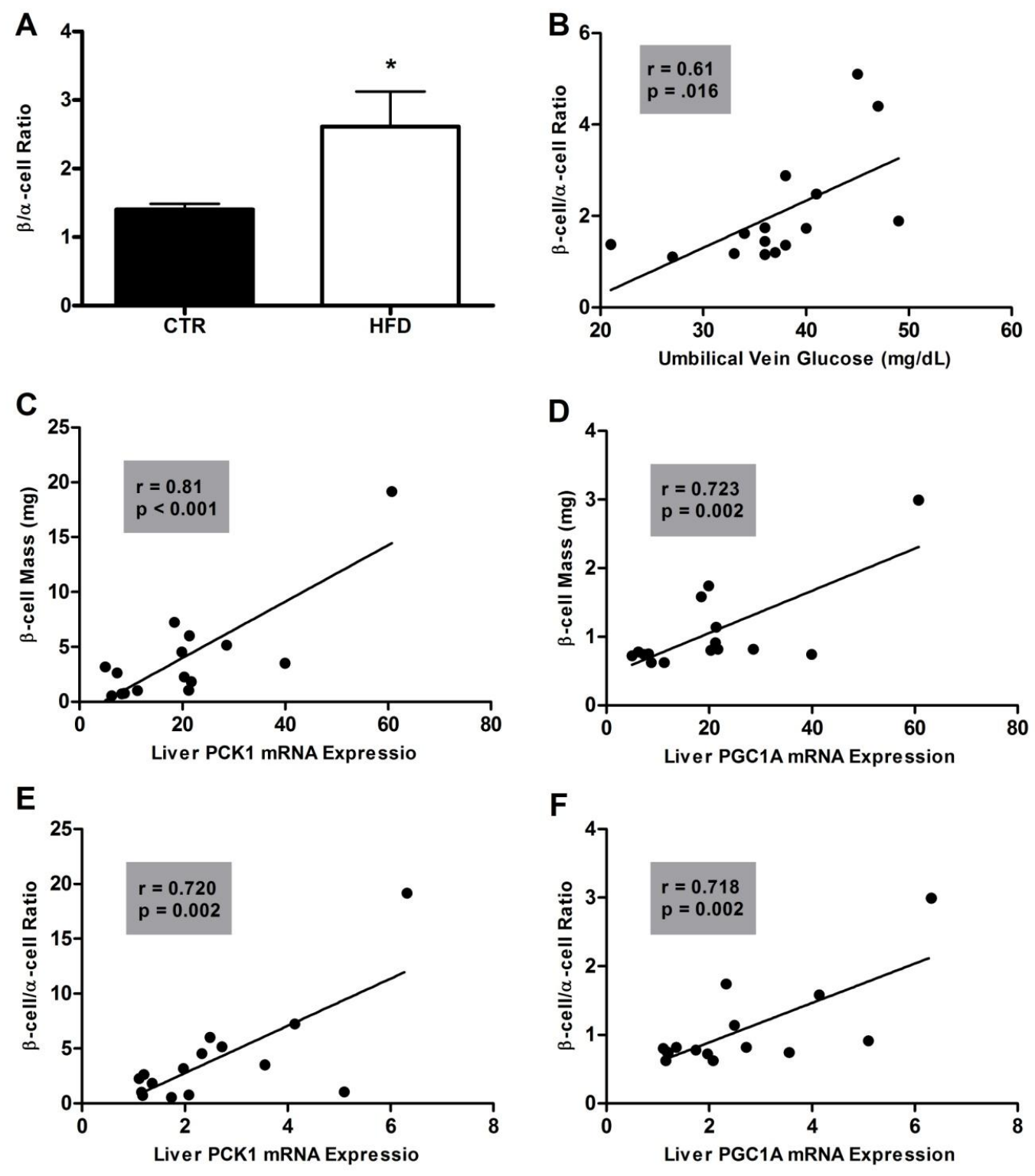

$\beta / \alpha$ cell ratio was increased in the HFD offspring $(\mathbf{A}) . * \mathrm{P}<0.05$ versus CTR (Student's ttest). CTR $n=7$, HFD $n=8$ There was a significant correlation between the $\beta / \alpha$ cell ratio and umbilical vein glucose $(\mathbf{B})$. Expression of $P C K 1(\mathbf{C}, \mathbf{E})$ and $P G C 1 A(\mathbf{D}, \mathbf{F})$ within the liver also significantly correlated with $\beta$ cell mass and $\beta / \alpha$ cell ratio. 
Figure 3.5: Transcription Factor Expression in Fetal Pancreas
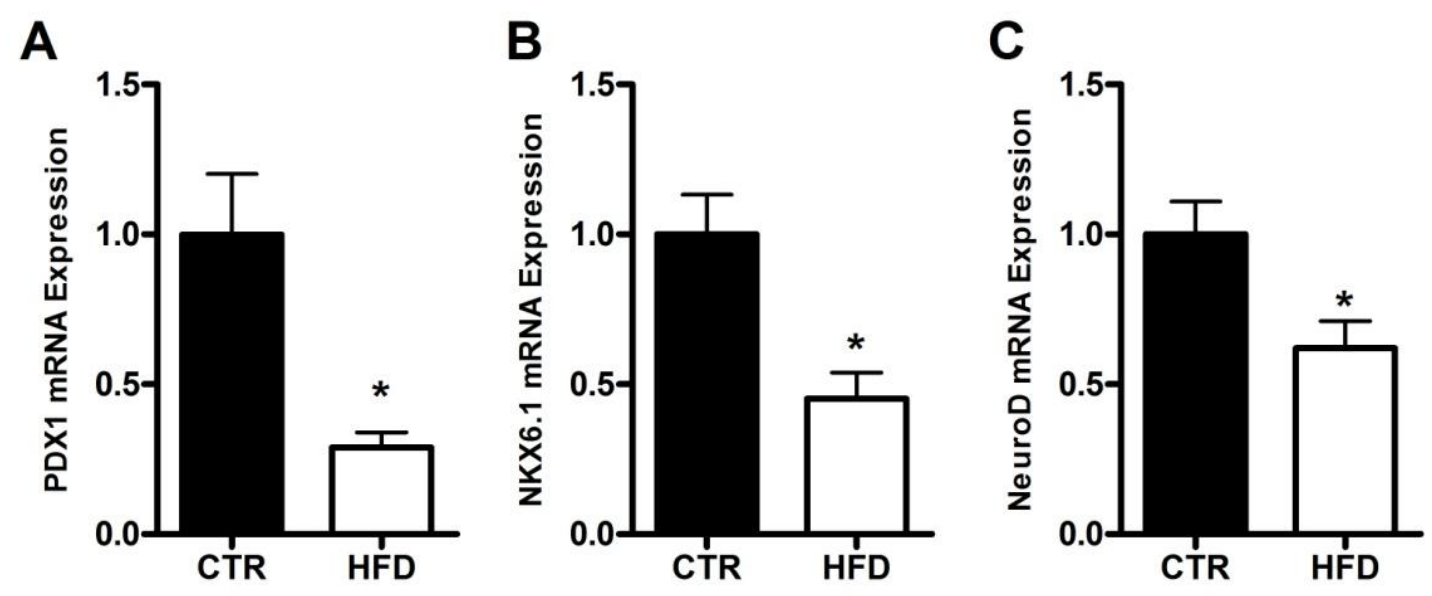

PDX1, NKX6.1 and NeuroD gene expression were significantly suppressed in the HFD offspring (A-C). ${ }^{*} \mathrm{P}<0.05$ versus CTR (Student's t-test). CTR $\mathrm{n}=12, \mathrm{HFD} \mathrm{n}=8$ 
Figure 3.6: Expression of Genes Involved in Glucose-stimulated Insulin Secretion in Fetal Pancreas
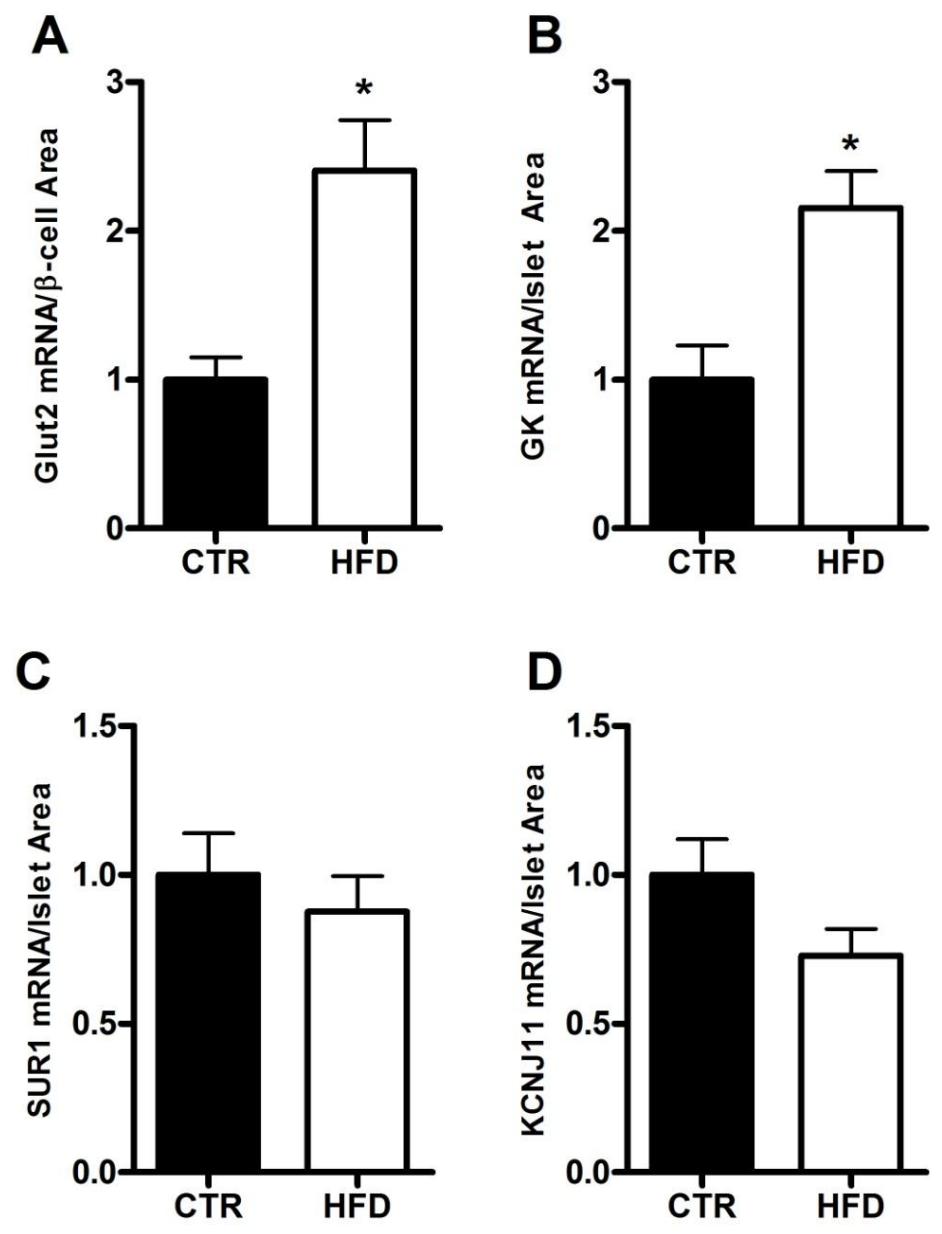

Glut2 was increased in the HFD offspring when normalized to $\beta$ cell area (A). Glucokinase was significantly elevated in the HFD offspring when normalized to islet area (B). SUR1 (C) and KCNJ11 (D) were not significantly affected by the HFD when normalized to islet area. ${ }^{*} \mathrm{P}<0.05$ versus CTR (Student's t-test). CTR $\mathrm{n}=12$; HFD $\mathrm{n}=$ 8 


\section{Chapter 3 Tables}

Comstock, 69 
Table 3.1: Comparison of CTR and HFD Fetuses

\begin{tabular}{|l|c|c|}
\hline Gestational Day 130 & Control & HFD \\
\hline $\mathrm{n}$ & 12 & 8 \\
\hline Weight $(\mathrm{g})$ & $350.2 \pm 8.0$ & $335.4 \pm 18.1$ \\
\hline Pancreas weight $(\mathrm{mg})$ & $256.0 \pm 16.6$ & $213.4 \pm 14.3$ \\
\hline Retro WAT Weight $(\mathrm{mg})$ & $120.8 \pm 23.0$ & $111.3 \pm 18.9$ \\
\hline Insulin $(\mu \mathrm{U} / \mathrm{mL})$ & $5.56 \pm 0.61$ & $5.92 \pm 0.06$ \\
\hline C-peptide $(\mathrm{ng} / \mathrm{mL})$ & $0.60 \pm 0.06$ & $0.44 \pm 0.04 *$ \\
\hline Glucose $(\mathrm{mg} / \mathrm{dL})$ & $116.8 \pm 12.2$ & $90.3 \pm 11.6$ \\
\hline Glucagon $(\mathrm{pg} / \mathrm{mL})$ & $126.5 \pm 16.6$ & $94.6 \pm 15.1$ \\
\hline Leptin $(\mathrm{ng} / \mathrm{mL})$ & $0.52 \pm 0.02$ & $0.51 \pm 0.01$ \\
\hline
\end{tabular}

Comstock, 70 
Table 3.2: Real-time RT-PCR Gene Expression in Fetal Pancreas

\begin{tabular}{|l|c|c|c|}
\hline \multicolumn{1}{|c|}{ Real-time PCR } & CTR & HFD & $\begin{array}{c}\text { HFD } \\
\text { (Normalized) }\end{array}$ \\
\hline $\mathrm{n}$ & 12 & 8 & 8 \\
\hline Glucagon & $1.00 \pm 0.12$ & $0.35 \pm 0.08^{*}$ & $0.72 \pm 0.08$ \\
\hline Glucokinase & $1.00 \pm 0.23$ & $1.45 \pm 0.25$ & $2.15 \pm 0.23^{*}$ \\
\hline Glut2 & $1.00 \pm 0.15$ & $1.62 \pm 0.34^{*}$ & $2.41 \pm 0.30^{*}$ \\
\hline HNF4 $\alpha$ & $1.00 \pm 0.11$ & $0.56 \pm 0.09 *$ & $0.83 \pm 0.12$ \\
\hline Insulin & $1.00 \pm 0.11$ & $0.32 \pm 0.07^{*}$ & $0.34 \pm 0.08 *$ \\
\hline KCNJ11/KIR6.2 & $1.00 \pm 0.12$ & $0.49 \pm 0.09 *$ & $0.49 \pm 0.09$ \\
\hline NeuroD & $1.00 \pm 0.11$ & $0.62 \pm 0.09 *$ & - \\
\hline NKX6.1 & $1.00 \pm 0.13$ & $0.45 \pm 0.09 *$ & - \\
\hline PDX1 & $1.00 \pm 0.20$ & $0.31 \pm 0.07 *$ & - \\
\hline SUR1 & $1.00 \pm 0.14$ & $0.59 \pm 0.12^{*}$ & $0.88 \pm 0.18$ \\
\hline
\end{tabular}

Comstock, 71 
Table 3.3: Comparison of CTR and HFD Dams

\begin{tabular}{|l|c|c|}
\hline Maternal Pregnancy Data & CTR & HFD \\
\hline $\mathrm{N}$ & 12 & 8 \\
\hline Weight $(\mathrm{Kg})$ & $8.7 \pm 0.48$ & $10.2 \pm 0.72$ \\
\hline Pregnancy Weight Gain $(\mathrm{Kg})$ & $0.30 \pm 0.23$ & $0.90 \pm 0.29$ \\
\hline Glucose $(\mathrm{mg} / \mathrm{dL})$ & $43.7 \pm 4.0$ & $42.9 \pm 2.3$ \\
\hline Insulin $(\mu \mathrm{U} / \mathrm{mL})$ & $19.7 \pm 3.7$ & $33.1 \pm 9.7$ \\
\hline Glucagon $(\mathrm{pg} / \mathrm{mL})$ & $110 \pm 16.5$ & $87.5 \pm 10.6$ \\
\hline Leptin $(\mathrm{ng} / \mathrm{mL})$ & $22.4 \pm 4.9$ & $55.5 \pm 12.6 *$ \\
\hline HOMA-IR & $2.4 \pm 0.65$ & $3.6 \pm 1.16$ \\
\hline IVGTT - Insulin AUC & $4157 \pm 599$ & $8183 \pm 1772 *$ \\
\hline IVGTT - Glucose AUC & $3905 \pm 207$ & $4018 \pm 253$ \\
\hline
\end{tabular}

Comstock, 72 
Chapter 4: High-Fat Diet During Perinatal Development Leads to Early Insulin Resistance in the Nonhuman Primate Infant 


\section{Introduction}

Since maternal HFD consumption is a significant contributor to the increased risk of MetS in children, it is essential to analyze the effects in our HFD post-natal offspring to determine what physiological processes may be leading up to the development of this syndrome. The purpose of these studies was to characterize the metabolic profile of juvenile Japanese macaques chronically exposed to a HFD. The decrease in $\alpha$ cell mass of fetal HFD offspring likely led to decreased paracrine regulation of $\beta$ cells, which resulted in dysfunction of insulin secretion. Due to this dysfunction, it is essential to determine if continued exposure to the HFD will significantly affect glucose homeostasis in the HFD offspring. I determined to analyze the physiology of the HFD animals up through weaning to determine if the islet effects measured in the HFD fetuses would translate to detrimental effects in infant glucose metabolism. I hypothesized that the decreased $\alpha$ cell mass and relative increase in the $\beta / \alpha$ cell ratio caused by the HFD during gestation would lead to perpetuation of the insulin secretory deficiency, but would ultimately result in increased insulin secretion and increased susceptibility of the HFD offspring to insulin resistance.

Comstock, 74 


\section{Materials and Methods}

Animals. All animal procedures were performed in accordance with the guidelines of the Institutional Animal Care and Use Committee of the ONPRC as previously described (Frias, et al.; Grant, et al.; Grayson, et al.; McCurdy, et al., 2009; Sullivan, et al., 2010) Briefly, age and weight matched adult female Japanese macaques were fed a control (CTR) or high-fat diet (HFD) for up to 5 years. Juvenile offspring were born naturally from CTR or HFD mothers during years 2 through 5 on the HFD. Physiological data collected from animals occurred during the first 210 days of life, prior to weaning.

Intravenous Glucose Tolerance Tests. We performed IVGTTs at P90 and P180 in the juveniles. In the dams we performed IVGTTs at 123 days ( \pm 5 days) gestation and at approximately 130 days ( \pm 6 days) postnatally. Animals were sedated with ketamine $(10 \mathrm{mg} / \mathrm{kg})$ and administered a glucose bolus (50\% dextrose solution) at a dose of 0.6 $\mathrm{g} / \mathrm{kg}$ via the saphenous vein. Baseline blood samples were obtained prior to the infusion, and blood samples were taken at $1,3,5,10,20,40$, and 60 min after infusion via the femoral artery. Glucose was measured immediately using a OneTouch Ultra2 Blood Glucose Monitor (LifeScan), and the remainder of the blood was kept in heparinized tubes on ice for insulin measurement. After IVGTT, samples were centrifuged, and plasma was stored with $5 \%$ aprotinin at $-80^{\circ} \mathrm{C}$ until assayed. Insulin was assayed in plasma by RIA (Millipore catalog no. RI-13K, Billerica, MA). HOMA-IR was calculated as fasting serum insulin $(\mu \mathrm{U} / \mathrm{ml}) \times$ fasting plasma glucose $(\mathrm{mmol} / \mathrm{l}) / 22.5$ as previously described (Bonora, et al., 2000; Matthews, et al., 1985). Baseline samples were assayed 
for glucagon (Millipore, catalog no. GL-32K, Billerica, MA), and leptin (Millipore catalog no. HL-81K, Billerica, MA).

Intravenous Insulin Tolerance Tests. We performed IVITTs at P210 on a subset of animals. Animals were sedated with telazol $(5 \mathrm{mg} / \mathrm{kg})$ and a baseline blood sample was collected and measured for glucose. The remainder of the blood was kept in a heparinized tube on ice for insulin measurement. Insulin was infused at $0.05 \mathrm{U} / \mathrm{Kg}$ and sequential blood samples were taken at 1, 3, 5, 10, 15, 20, 25 and 30 minutes. After IVITT, the initial baseline sample was centrifuged, and plasma was stored with 5\% aprotinin at $-80^{\circ} \mathrm{C}$ until assayed. Insulin was assayed in plasma by RIA (Milllipore catalog no. RI-13K, Billerica, MA). The insulin sensitivity index (ISI) was calculated as the change in glycemia from baseline to 15 minutes (estimated using the linear regression from baseline to 15 minutes) divided by the initial glycemia (average of both baselines) as previously described (Grulet, Durlach, Hecart, Gross, \& Leutenegger, 1993). 


\section{Results}

Physiology of P30 and P90 Offspring. At postnatal day 30 (P30), body weight was not significantly different between diet groups, although the HFD offspring did have increased adiposity (Fig. 4.1a\& c). Also, males were significantly heavier than females at P30 (Fig. 4.1b). Body mass index (BMI) was significantly decreased in the HFD offspring at P30 (Fig. 4.1d), indicating that the intra-uterine HFD exposure lead to growth restriction in these animals.

At P90 males were still heavier than females (Fig. 4.2a). However, females tended to have increased body fat (Table 4.1). Glucose was not significantly affected by the diet; however, at P90 males did secrete approximately 15\% more glucose than females (Fig. 4.2b). Also, insulin response to an IVGTT was significantly suppressed in the HFD offspring (Fig. 4.2c). The decreased insulin secretion at P90 is likely due to an increase in insulin secretion in the HFD mothers during lactation (Fig. 4.2d), as insulin is passed into and absorbed from the breast milk (Jovanovic-Peterson, Fuhrmann, Hedden, Walker, $\&$ Peterson, 1989). Previous publications from this lab have demonstrated an increase in the insulin content of the breast milk of HFD mothers (Grant, et al., 2011).

Physiology of P180 and P210 Offspring. By P180 HFD offspring were significantly heavier (Fig. 4.3a) and the sex effect was no longer demonstrable (Table 4.1). While still very lean, the HFD animals had elevated body fat (Fig. 4.3b). At P180 HFD offspring insulin secretion was normalized to control levels (Fig. 4.3c). At P210, 
just prior to weaning, animals on the HFD had become insulin resistant as indicated by a $30 \%$ decrease in their insulin sensitivity index (Fig. 4.3d). 


\section{Discussion}

In order to determine if fetal changes in islet composition and gene expression might lead to lasting physiological effects in infant macaques from HFD dams, I studied the physiology of these animals after birth until weaning. Infant offspring consume only maternal milk during the first three months of life. Since previous studies from our lab have shown no significant difference in total fatty acid content in milk from dams on the CTR versus HFD, it is likely that they will not be exposed to the specific metabolic pressure of the HFD until after P90. In fact, at P90, we demonstrated decreased insulin secretion in the HFD offspring which may be reflective of the high insulin content of the mother's milk. However, since insulin secretion is clearly affected in the HFD fetal offspring, this may reflect the decreased paracrine regulation of HFD fetal islets. By 7 months of age, the HFD animals were heavier, had increased body fat and were insulin resistant. However, at this time, there was no significant difference in fasting insulin or insulin response to an IVGTT. This indicates that, while HFD exposure during the early post-natal period may lead to whole body insulin resistance. While islet defects were not measureablel at this stage if $\alpha$ cell mass remained decreased, it may produce a beneficial effect in the HFD post-natal offspring which will lead to decreased glucagon production and allow them to adapt to the high lipid content of the diet.

Although HFD during fetal development may program the fetal pancreas to withstand the early postnatal insults of this diet, this adaptation may not continue when the juvenile offspring are chronically exposed to the increased metabolic pressure that the HFD produces. As we have previously demonstrated, there are significant effects of the 
HFD on liver function and morphology in the fetuses of HFD mothers (Grant, et al., 2011; McCurdy, et al., 2009), indicating that insulin resistance in the HFD animal results from dysfunction of peripheral tissues first and then hyperinsulinemia will occur as a response to this resistance. While insulin hyper-secretion and insulin resistance occur concurrently in the diabetic adult, in this model we have demonstrated that the pathway to disease progression starts with insulin resistance. My hypothesis that decreased $\alpha$ cell mass and relative increase in the $\beta / \alpha$ cell ratio caused by the HFD during gestation leads to elevated insulin secretion is not supported by this data. The decreased insulin secretion at P90 may be a result of $\beta$ cell dysfunction; however, based on the limitations of this study, it is not possible to determine how islet composition is affected. Yet, these animals become insulin-resistant, as I hypothesized. It is essential to follow these animals until a point at which there are significant physiologic measures of islet dysfunction in order to determine how islets adapt in the juvenile animal to a HFD. 
Chapter 4 Figures

Comstock, 81 
Figure 4.1: P30 Physiology
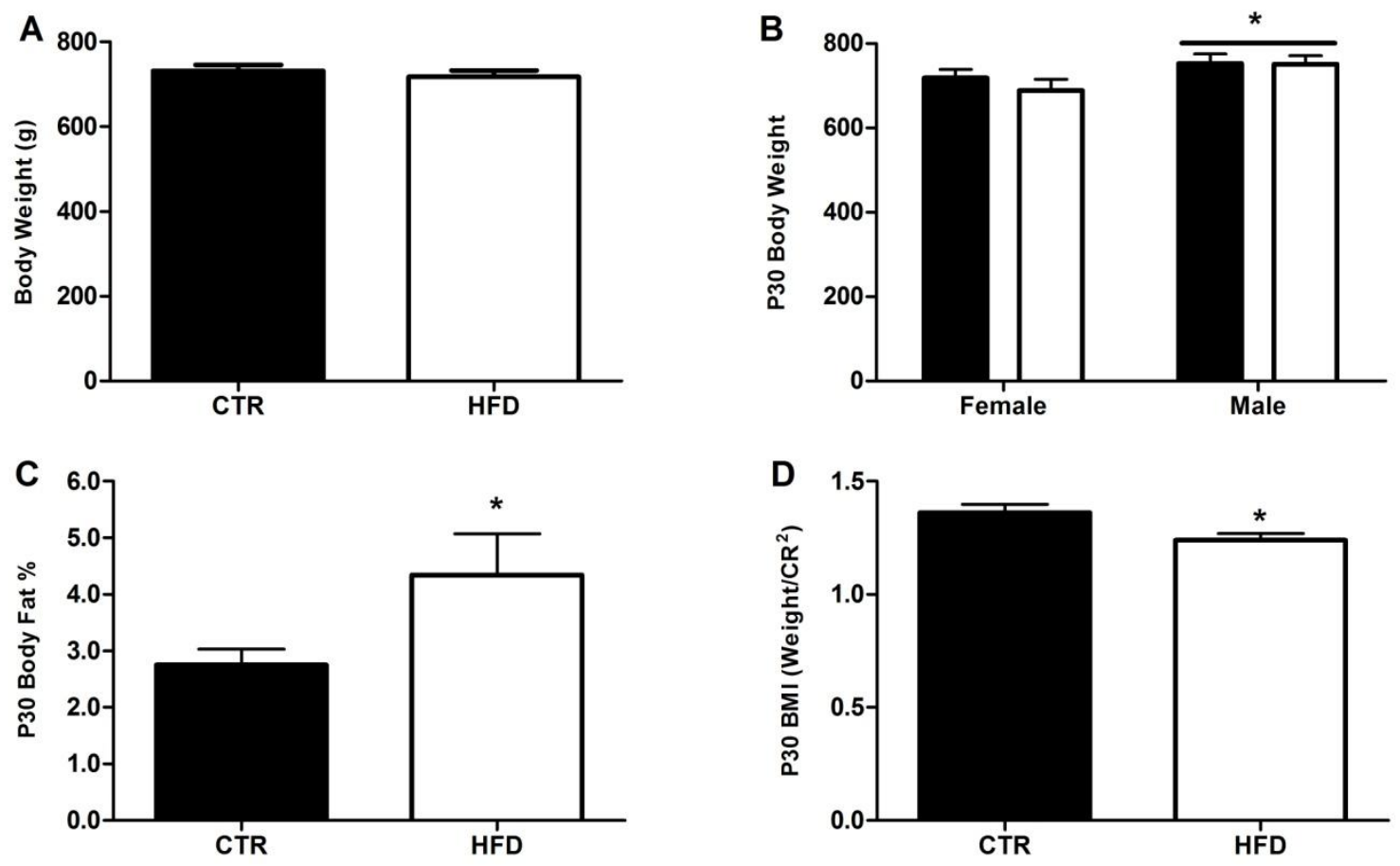

Body weight was not significantly affected by the HFD at P30 (A). However, males were significantly heavier than females (B). Body fat was significantly elevated in the HFD offspring (C). BMI was significantly decreased in HFD offspring (D). 
Figure 4.2: P90 Physiology and Maternal IVGTT IAUC
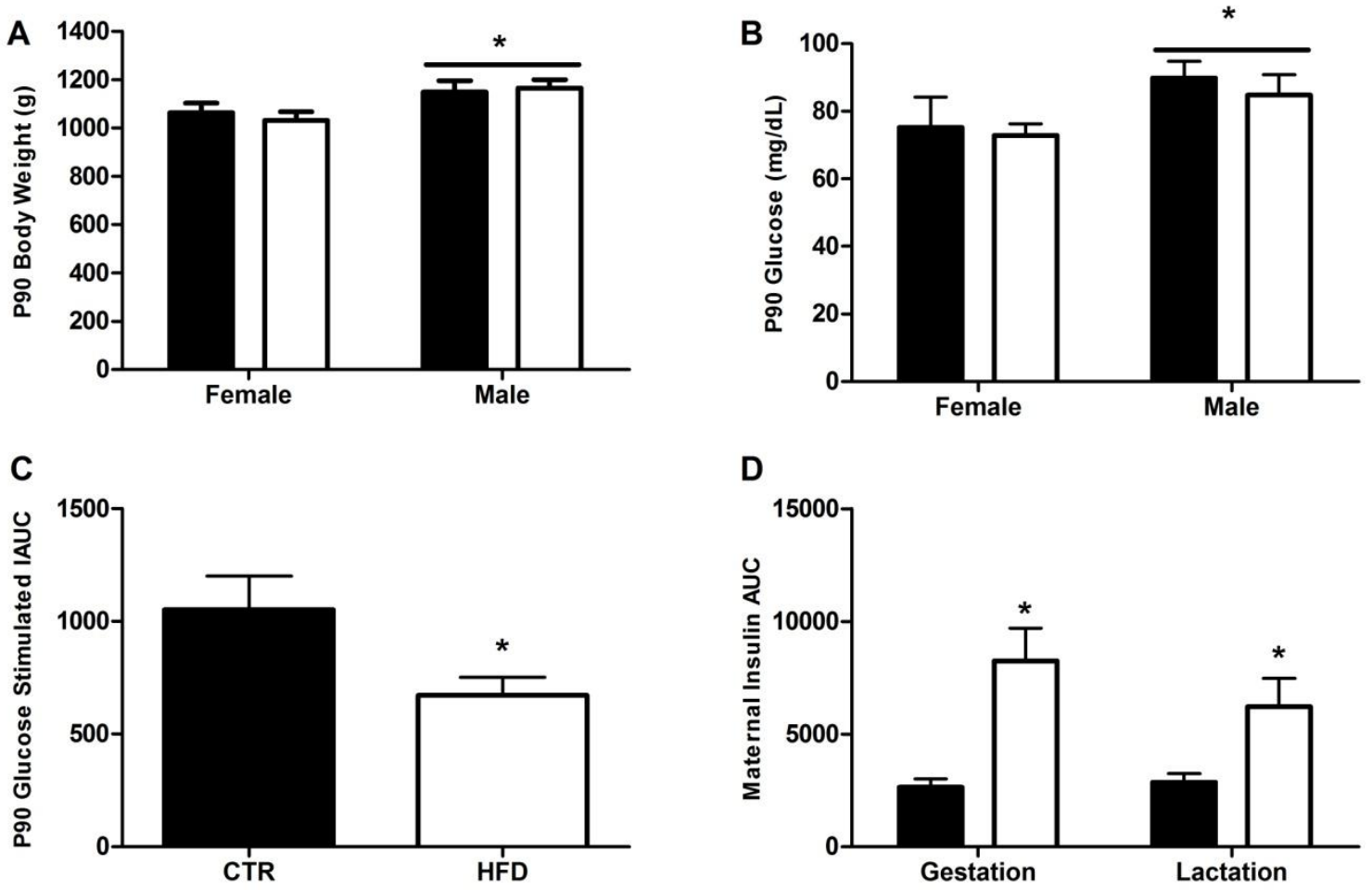

At P90 males were still significantly heavier than females (A). Fasting glucose was also elevated in males (B). IVGTT insulin AUC was decreased in HFD offspring (C). Maternal IVGTT insulin AUC was elevated during gestation and lactation (D). 
Figure 4.3: P180 and P210 Physiology
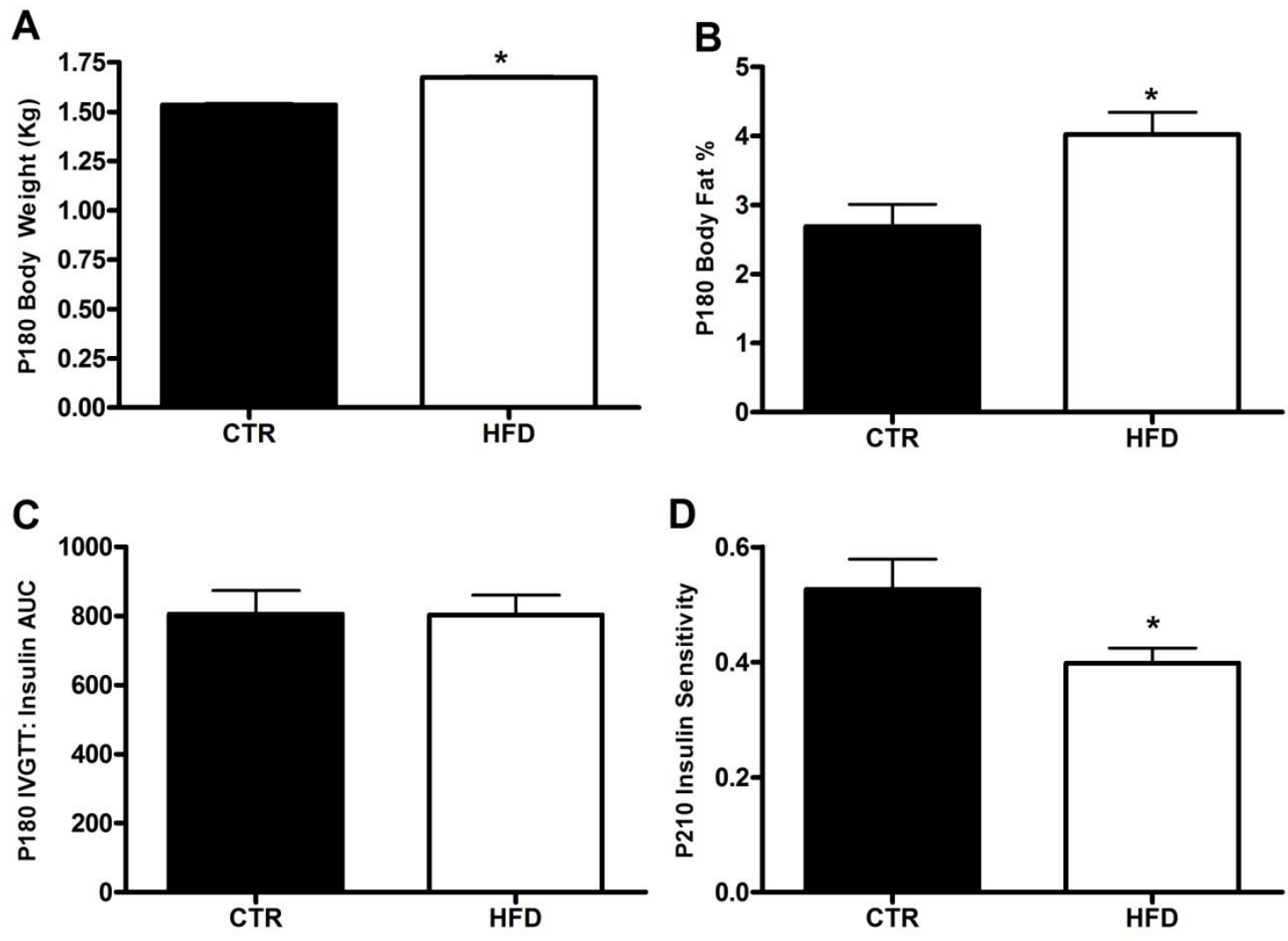

Body weight was significantly increased in the HFD offspring by P180 (A). Body fat percentage was significantly elevated in the P180 offspring (B). There was no significant effect of HFD on insulin secretion during a GTT at P180 (C). ${ }^{*} \mathrm{P}<0.05$ versus CTR (Student's t-test) CTR n=24, HFD n= 35. By P210 HFD animals are insulin resistant (D). $* \mathrm{P}<0.05$ versus CTR (Student's t-test; CTR $\mathrm{n}=16, \mathrm{HFD} \mathrm{n}=14$ ) 
Chapter 4 Tables

Comstock, 85 
Table 4.1: Physiology of P30 and P90 Offspring

\begin{tabular}{|c|c|c|c|c|c|}
\hline Sample Size & 10 & 14 & 16 & 19 & \multirow{3}{*}{$\begin{array}{c}\text { Diet or } \\
\text { Sex } \\
\text { Effect }\end{array}$} \\
\hline Sex & Female & Male & Female & Male & \\
\hline Postnatal Day 30 & \multicolumn{2}{|c|}{ CTR } & \multicolumn{2}{|c|}{ HFD } & \\
\hline Weight (g) & $719 \pm 19.8$ & $753 \pm 22.1$ & $688 \pm 27.6$ & $751 \pm 20.1$ & SEX \\
\hline Ponderal Index & $57.5 \pm 2.95$ & $56.7 \pm 2.24$ & $55.7 \pm 1.94$ & $56.9 \pm 2.93$ & - \\
\hline Total Body Fat \% & $2.81 \pm 0.44$ & $2.69 \pm 0.35$ & $5.63 \pm 1.32$ & $3.42 \pm 0.57$ & DIET \\
\hline Postnatal Day 90 & \multicolumn{2}{|c|}{ CTR } & \multicolumn{2}{|c|}{ HFD } & \\
\hline Weight (g) & $1064 \pm 40.3$ & $1149 \pm 46$ & $1031 \pm 36$ & $1165 \pm 35$ & SEX \\
\hline Ponderal Index & $5.79 \pm 0.24$ & $5.9 \pm 0.19$ & $5.6 \pm 0.2$ & $5.6 \pm 0.2$ & - \\
\hline Body Mass Index & $1.51 \pm 0.04$ & $1.59 \pm 0.04$ & $1.48 \pm 0.05$ & $1.53 \pm 0.05$ & SEX \\
\hline Total Body Fat \% & $4.85 \pm 0.65$ & $4.38 \pm 0.60$ & $5.99 \pm 0.54$ & $5.3 \pm 0.47$ & - \\
\hline Fasting Glucose (mg/dL) & $75.2 \pm 9.0$ & $89.9 \pm 4.9$ & $72.8 \pm 3.5$ & $84.8 \pm 6.0$ & SEX \\
\hline Fasting Insulin $(\mu \mathrm{U} / \mathrm{mL})$ & $13.9 \pm 4.7$ & $14.1 \pm 4.5$ & $10.4 \pm 1.87$ & $10.0 \pm 2.48$ & - \\
\hline Glucagon $(\mathrm{pg} / \mathrm{mL})$ & $103.4 \pm 36.6$ & $110.9 \pm 13.6$ & $124.85 \pm 25.5$ & $110.5 \pm 12.0$ & - \\
\hline Leptin (ng/mL) & $2.37 \pm 0.52$ & $2.20 \pm 0.30$ & $2.52 \pm 0.34$ & $2.02 \pm 0.31$ & - \\
\hline HOMA-IR & $4.27 \pm 1.31$ & $4.18 \pm 1.02$ & $1.97 \pm 0.42$ & $2.44 \pm 0.89$ & - \\
\hline IVGTT - GSIS & $1179 \pm 260$ & $958 \pm 186$ & $804 \pm 158$ & $579 \pm 95$ & DIET \\
\hline IVGTT - Glucose AUC & $3712 \pm 591$ & $3802 \pm 412$ & $3416 \pm 274$ & $3788 \pm 211$ & - \\
\hline IVGTT - Total Insulin AUC & $1663 \pm 228$ & $1953 \pm 278$ & $1318 \pm 213$ & $1286 \pm 154$ & DIET \\
\hline IVGTT - 1st Phase Insulin & $15.7 \pm 3.43$ & $19 \pm 4.0$ & $22.0 \pm 5.5$ & $18.9 \pm 3.4$ & - \\
\hline IVGTT - 2nd Phase Insulin & $1493 \pm 201$ & $1837 \pm 262$ & $1211 \pm 93$ & $1166 \pm 142$ & - \\
\hline
\end{tabular}

Physiological measurements of infantile offspring taken at post-natal day 30 and 90 . Diet or sex effect indicated on table, $\mathrm{P}<0.05$ ( 2 way ANOVA, with Bonferroni's post-hoc test). 
Table 4.2: Physiology of P180 and P210 Offspring

\begin{tabular}{|c|c|c|c|c|c|}
\hline Postnatal Day 180 & \multicolumn{2}{|c|}{ CTR } & \multicolumn{2}{|c|}{ HFD } & \multirow[b]{2}{*}{ DIET } \\
\hline Weight (g) & $1591 \pm 54$ & $1685 \pm 85$ & $1576 \pm 56$ & $1745 \pm 63$ & \\
\hline Body Mass Index & $1.66 \pm 0.09$ & $1.78 \pm 0.11$ & $1.72 \pm 0.70$ & $1.86 \pm 0.79$ & - \\
\hline Total Body Fat \% & $4.10 \pm 0.61$ & $1.53 \pm 0.70$ & $4.22 \pm 0.46$ & $3.83 \pm 0.52$ & DIET \\
\hline Fasting Glucose (mg/dL) & $86 \pm 6.0$ & $86 \pm 6.9$ & $75 \pm 4.5$ & $90 \pm 5.1$ & - \\
\hline Fasting Insulin $(\mu \mathrm{U} / \mathrm{mL})$ & $15.7 \pm 4.9$ & $17.4 \pm 5.7$ & $18.7 \pm 3.7$ & $17.3 \pm 4.2$ & - \\
\hline Glucagon (pg/mL) & $104 \pm 24.2$ & $122 \pm 26.8$ & $142 \pm 17.6$ & $159.6 \pm 19.8$ & - \\
\hline Leptin (ng/mL) & $2.60 \pm 0.59$ & $2.34 \pm 0.68$ & $3.08 \pm 0.45$ & $1.99 \pm 0.51$ & - \\
\hline HOMA-IR & $3.41 \pm 1.17$ & $3.74 \pm 1.36$ & $3.71 \pm 0.89$ & $4.10 \pm 1.00$ & - \\
\hline IVGTT - GSIS & $729 \pm 137$ & $752 \pm 158$ & $850 \pm 103$ & $712 \pm 117$ & - \\
\hline IVGTT - Glucose AUC & $3502 \pm 468$ & $3729 \pm 541$ & $3750 \pm 354$ & $3613 \pm 400$ & - \\
\hline IVGTT - Total Insulin AUC & $1690 \pm 230$ & $1730 \pm 266$ & $1824 \pm 174$ & $1869 \pm 196$ & - \\
\hline IVGTT - 1st Phase Insulin & $25.8 \pm 4.4$ & $15.3 \pm 5.1$ & $22.7 \pm 3.3$ & $21.4 \pm 3.8$ & - \\
\hline IVGTT - 2nd Phase Insulin & $1514 \pm 220$ & $1592 \pm 242$ & $1665 \pm 159$ & $1701 \pm 179$ & - \\
\hline Postnatal Day 210 & \multicolumn{2}{|c|}{ CTR } & \multicolumn{2}{|c|}{ HFD } & \\
\hline Sample Size & 7 & 9 & 9 & 5 & \\
\hline Weight (g) & $1771 \pm 64$ & $1794 \pm 66$ & $1824 \pm 42$ & $1956 \pm 112$ & DIET \\
\hline Fasting Glucose (mg/dL) & $83 \pm 12.4$ & $86 \pm 5.7$ & $79 \pm 10.4$ & $73 \pm 4.4$ & - \\
\hline Fasting Insulin $(\mu \mathrm{U} / \mathrm{mL})$ & $23.4 \pm 4.5$ & $18.1 \pm 4.7$ & $24.2 \pm 8.4$ & $9.8 \pm 3.3$ & SEX \\
\hline ITT Insulin Sensitivity Index & $0.54 \pm 0.08$ & $0.62 \pm 0.08$ & $0.45 \pm 0.06$ & $0.39 \pm 0.05$ & DIET \\
\hline
\end{tabular}

Physiological measurements of infantile offspring taken at post-natal day 180 and 210.

Diet or sex effect indicated on table, $\mathrm{P}<0.05$ ( 2 way ANOVA, with Bonferroni's posthoc test). 
Table 4.3: Physiology of Dams of Infant Offspring

\begin{tabular}{|c|c|c|c|c|c|}
\hline Sample Size & 10 & 14 & 16 & 19 & \multirow{3}{*}{$\begin{array}{c}\text { Diet or } \\
\text { Sex } \\
\text { Effect }\end{array}$} \\
\hline Offspring Sex & Female & Male & Female & Male & \\
\hline Maternal Pregnancy Data & \multicolumn{2}{|c|}{ CTR } & \multicolumn{2}{|c|}{ HFD } & \\
\hline Weight (Kg) & $8.9 \pm 1.1$ & $9.7 \pm 1.4$ & $11.8 \pm 1.7$ & $11.9 \pm 2.2$ & DIET \\
\hline Pregnancy Weight Gain (Kg) & $0.23 \pm 0.4$ & $0.72 \pm 0.3$ & $0.28 \pm 0.2$ & $1.4 \pm 0.7$ & - \\
\hline Glucose (mg/dL) & $43 \pm 4.2$ & $42 \pm 2.8$ & $39 \pm 2.8$ & $48 \pm 3.6$ & - \\
\hline Insulin $(\mu \mathrm{U} / \mathrm{mL})$ & $10.0 \pm 2.2$ & $11.5 \pm 2.7$ & $40.6 \pm 6.4$ & $63.9 \pm 7.5$ & DIET \\
\hline HOMA-IR & $1.1 \pm 0.2$ & $1.3 \pm 0.3$ & $4.2 \pm 1.1$ & $8.5 \pm 2.2$ & DIET \\
\hline IVGTT - Insulin AUC & $2725 \pm 49$ & $2388 \pm 30$ & $6406 \pm 67$ & $10493 \pm 106$ & DIET \\
\hline IVGTT - Glucose AUC & $4791 \pm 34$ & $5341 \pm 25$ & $4575 \pm 39$ & $4320 \pm 30$ & - \\
\hline Maternal Lactation Data & \multicolumn{2}{|c|}{ CTR } & \multicolumn{2}{|c|}{ HFD } & \\
\hline Weight (Kg) & $9.3 \pm 0.6$ & $9.5 \pm 0.5$ & $12.7 \pm 0.7$ & $11.9 \pm 0.5$ & DIET \\
\hline Glucose (mg/dL) & $53 \pm 3.7$ & $51 \pm 2.1$ & $55 \pm 1.7$ & $53 \pm 2.0$ & - \\
\hline Insulin $(\mu \mathrm{U} / \mathrm{mL})$ & $15.5 \pm 5.0$ & $16.0 \pm 2.9$ & $56.8 \pm 19.7$ & $32.5 \pm 8.4$ & DIET \\
\hline HOMA-IR & $2.3 \pm 0.8$ & $2.0 \pm 0.4$ & $7.4 \pm 2.4$ & $5.0 \pm 1.1$ & DIET \\
\hline IVGTT - Insulin AUC & $3242 \pm 926$ & $2819 \pm 397$ & $6157 \pm 754$ & $4195 \pm 758$ & DIET \\
\hline IVGTT - Glucose AUC & $6199 \pm 622$ & $6260 \pm 329$ & $6113 \pm 338$ & $5539 \pm 396$ & - \\
\hline
\end{tabular}

Physiological measurements of dams of infant offspring taken during the third trimester of pregnancy and during the $3-4^{\text {th }}$ month of lacation, separated by sex of the offspring. Diet or sex effect indicated on table, $\mathrm{P}<0.05$ ( 2 way ANOVA, with Bonferroni's posthoc test). 
Chapter 5: Chronic High-Fat Diet Consumption During Pregnancy and the Early Postnatal Period Leads to Decreased $\alpha$ Cell Plasticity in the Juvenile Nonhuman Primate 


\section{Introduction}

In the previous chapters, I have shown that maternal obesity and/or HFD consumption can impact fetal islet development and lead to insulin resistance in the postnatal offspring prior to weaning. Specifically, I demonstrated a decrease in $\alpha$ cell mass and a down-regulation of transcription factors involved in the differentiation pathway of these cells during pregnancy(Comstock, 2012). In contrast, in rodent models, $\alpha$ cell mass is increased in response to a HFD (Cerf, et al., 2005). However, in sheep models, there is only evidence of $\beta$ cell abnormalities when exposed to maternal overnutrition during the

second trimester of gestation (S. P. Ford, et al., 2009; L. Zhang, et al., 2011). While many studies have been performed on post-natal development in the rodent, there are significant species differences. Furthermore, in primate species, there is significant islet expansion through puberty (Hansen \& Bodkin, 1990; Wolfe-Coote, Louw, Woodroof, \& Du Toit, 1996). Therefore, post-natal nutrition may also significantly impact islet development and function.

The primary purpose of these studies was to determine the relative impact of early programming events (maternal diet) versus post-weaning dietary influence on pancreas development and potential long-term susceptibility to diabetes. To accomplish this, I compared islet development and function in juvenile macaques from four different groups: CTR/CTR, CTR/HFD, HFD/CTR and HFD/HFD. Using these four groups, we can determine the relative impact of maternal HFD versus the impact of the HFD during the post-weaning period on islet structural morphology and function. I hypothesized that the decreased $\alpha$ cell mass in the HFD offspring would perpetuate by continued HFD 
exposure through the post-weaning period and would lead to hyperinsulinemia, increased insulin resistance, and persistant changes in islet morphology. My second hypothesis was that a post-weaning dietary intervention to a standard control (CTR) diet could reverse the effects of HFD consumption during early development (HFD/CTR). Finally, my third hypothesis was that post-weaning HFD alone (CTR/HFD) would lead to alterations in islet morphology that would have diminished effects on glucose homeostasis, compared to the HFD/HFD offspring. 


\section{Materials and Methods}

Animals. All animal procedures performed were in accordance with the guidelines of the Institutional Animal Care and Use Committee of the Oregon National Primate Research Center (ONPRC) as previously described (Frias, et al.; Grant, et al.; Grayson, et al.; McCurdy, et al., 2009; Sullivan, et al.). Briefly, age- and weight-matched adult female Japanese macaques were fed a CTR or HFD. Juvenile offspring were born naturally from CTR or HFD mothers during years 2 through 5 on the HFD and allowed to stay with their mothers until weaning at approximately 8 months of age ( $249+/-4$ days).

At 8 months, the juveniles were weaned and maintained on the same diet or switched to the opposing diet, for 5 months $(143+/-3$ days $)$, thus generating four juvenile groups: CTR/CTR, CTR/HFD, HFD/CTR \& HFD/HFD. These animals were necropsied at approximately 13 months of age $(393+/-4$ days $)$.

Intravenous Glucose Tolerance Tests. As previously described (Grant, et al., 2011; McCurdy, et al., 2009), we performed an IVGTT 1 week prior to necropsy. Briefly, after a 5 hour morning fast, animals are sedated with ketamine and administered a glucose bolus at a dose of $0.6 \mathrm{~g} / \mathrm{kg}$ via the saphenous vein. Baseline blood samples were obtained prior to the infusion, and samples were taken at $1,3,5,10,20,40$, and 60 minutes after infusion via the femoral artery. Glucose was measured immediately and the remainder of the blood was kept on ice for insulin measurement in plasma by RIA (catalog no. RI-13K; Linco). HOMA-IR was calculated as fasting serum insulin $(\mu \mathrm{U} / \mathrm{ml})$ $\times$ fasting plasma glucose $(\mathrm{mmol} / \mathrm{l}) / 22.5$, as previously described (Bonora, et al., 2000; Matthews, et al., 1985).

Comstock, 92 
RNA Isolation and Quantitative RT-PCR. Total RNA was isolated from 5 head-to-tail grouped sections of each offspring pancreas (Chapter 3, Fig. 3.1) using TRIzol reagent (Invitrogen, Carlsbad, CA). RNA was DNase-treated and reversetranscribed. mRNA was measured by real-time quantitative RT-PCR using a PE Applied Biosystems PRISM 7700 Sequences Detector System (Invitrogen Life Technologies, Carlsbad, CA). Premade qPCR gene expression assays directed against human sequences were purchased from Applied Biosystems. The amplification was performed as follows: 2 min at $50^{\circ} \mathrm{C}, 10 \mathrm{~min}$ at $95^{\circ} \mathrm{C}$, then 45 cycles each at $95^{\circ} \mathrm{C}$ for $15 \mathrm{sec}$ and at $60^{\circ} \mathrm{C}$ for 60 sec in the ABI/Prism 7700 Sequences Detector System. Standard curves on serial dilutions of the cDNA $(1: 10,1: 50,1: 1001: 500,1: 1000$ and 1:10,000) were performed using cDNA from pooled pancreas samples. Standard curves were drawn on the basis of the log of the input RNA versus the critical threshold (CT) cycle, in which the fluorescence of the sample is greater than the threshold of the baseline fluorescence. The housekeeping gene $\beta$-actin was assayed and shown to be consistently expressed between juvenile groups, while two other commonly used genes (18S and GAPDH) were significantly differentially expressed in the various diet groups. Therefore, all qRT-PCR results are normalized to $\beta$-actin expression.

Immunohistochemistry. Pancreas tissue was collected from 5 head-to-tail areas (Chapter 3, Fig. 3.1) and cut into $5 \mu \mathrm{m}$ paraffin-embedded sections. Sections were deparaffinized in xylene and then washed with 100\%, 95\%, 85\%, and 70\% graded ethanol for 5 minutes each. Microwaving in citrate buffer was used for antigen retrieval. Standard immunohistochemical methods were used. Briefly, sections were washed in 
KPBS and then blocked in $2 \%$ donkey serum in $0.4 \%$ triton X/KPBS. Antibodies against insulin (\#20056, 1:2000, Immmunostar, Hudson, WI) and glucagon (\#4031-01F, 1:10,000, Linco, St. Charles, MO) were used, which have been previously described for immunohistochemical identification of $\beta$ cells and $\alpha$ cells, respectively (Elghazi, CrasMeneur, Czernichow, \& Scharfmann, 2002; Prado, Pugh-Bernard, Elghazi, Sosa-Pineda, \& Sussel, 2004). Primary antibodies were diluted in $2 \%$ donkey serum in $0.4 \%$ triton $\mathrm{X} / \mathrm{KPBS}$ and applied to tissue sections where they were incubated overnight. Tissues were then washed in KPBS and secondary antibodies were applied for 1 hour at room temperature at a dilution of 1:200 (Jackson ImmunoResearch Laboratories, Inc, West Grove, PA). The specificity of the antibodies were determined by omitting the primary and using only the secondary. These tests did not produce staining.

Quantitative Analysis. Images were acquired with a MarianasTM imaging workstation (Intelligent Imaging Innovations, Denver, CO), using a 20X objective and the stereology module to montage images covering whole sections. Areas occupied by $\beta$ and $\alpha$ cells were measured based on intensity segmentation of each fluorescence channel using Slidebook 5.0. The $\beta$ and $\alpha$ cells fractions were calculated by dividing by the area of the whole section. Total islet area (insulin and glucagon stained area/DAPI stained area), islet mass (total islet area $\mathrm{x}$ pancreas weight), $\beta$ and $\alpha$ cell mass [(total insulin or glucagon stained area/DAPI stained area) x pancreas weight] and islet density (total islet number/DAPI area) were calculated as previously reported (Uchida, et al., 2005). Islets were classified based on size: stained cell clusters with an area greater than $1000 \mu \mathrm{m}^{2}$ or diameter approximately $35 \mu \mathrm{m}$ were arbitrarily defined as large islets, while any clusters 
smaller than this were classified as small islets. Individual $\alpha$ and $\beta$ cell size and number were quantified in a manner similar to that previously described (Beringue, et al., 2002; Montanya \& Tellez, 2009). Briefly, stereologically sampled 20x images from the tail region of each pancreas were selected, and 3 to 5 whole islets were quantified from each animal. Individual $\alpha$ and $\beta$ cell area was measured based on glucagon and insulin staining and the number of nuclei were quantified in that area.

Data Analysis. For all analyses not specifically described above, data were compiled and tested for normality with SPSS Statistics 19 software (Armonk, New York). Data was tested for maternal or post-weaning diet effect using two-way ANOVA, then Bonferroni post-hoc analysis to test difference between individual groups. In order to determine a sex effext, a three-way ANOVA was performed on all data for sex, maternal diet and post-weaning diet. Significance was reported as $\mathrm{p}<0.05$. All graphs were made with Prism software (GraphPad Software, Inc., La Jolla, CA). 


\section{Results}

Metabolic Characteristics of Juvenile Offspring Exposed to a HFD. During the post-weaning period, both CTR/HFD and HFD/HFD animals gain significantly more weight than CTR/CTR animals (data not shown). However, by 13 months, only the HFD/HFD animals were significantly heavier than CTR/CTR animals (Fig. 5.1a). Maternal diet did not significantly affect final weight; rather, the post-weaning diet was the most significant predictive of weight at necropsy. Interestingly, for the HFD/HFD and HFD/CTR groups, males had a higher (approximately 10\% higher) final weight than females; in contrast, for the CTR/CTR and CTR/HFD, the final weights were similar for males and females (Fig. 5.2a). While all animals exposed to HFD diet tended to have slightly elevated overall body fat (as determined by DEXA); this was not significantly different (Table 5.1). However, retroperitoneal white adipose tissue (RWAT) mass was elevated in both post-wean fed HFD groups (Fig. 5.1b; Table 5.1), and subscapular caliper measures, an indicator of subcutaneous fat mass, were also significantly greater in these groups (not shown). It should be recognized that, as a whole, these animals are very lean at 13 months, with less that $3 \%$ body fat (Table 5.1). Consistent, with the sexdependent differences in weight, fasting glucose was significantly elevated specifically in the males that had been exposed to the maternal diet (HFD/HFD and HFD/CTR), but was not significantly different between the female diet groups (Fig. 4.2b). In contrast, fasting insulin, insulin area under the curve (IAUC) and HOMA-IR was similarly increased in both sexes in response to the post-weaning high-fat diet exposure (Fig. 5.1c\&d, Table 
5.1); however, this was only significant in the HFD/HFD group. There was no significant difference in basal glucagon levels between the different groups (Table 5.1).

Altered Islet Morphology in HFD Juveniles. Stereological analysis of pancreatic islets was performed to determine the relative contribution of islet mass, size, density and islet composition upon the physiological effects noted in the juveniles. Importantly, pancreas weight was lower specifically in the CTR/HFD group (Table 5.1), thus all subsequent measures were normalized to pancreas weight. Islet mass of the both CTR/HFD and HFD/HFD animals was increased by $30 \%$ when compared to CTR/CTR, while the HFD/CTR was not significantly different from CTR/CTR (Fig 5.3a). Interestingly, the increase in islet mass in the HFD/HFD group was due to an increase in the average islet diameter (Fig. 5.3b). However, the increase in the CTR/HFD was due to an increase in islet density (Fig. 5.3c). Further analysis of islets by size demonstrated an increase in large islet mass in the HFD/HFD group and an increase in small islet density in the CTR/HFD group (Fig. 5.4a\&c). In order to determine which cell type contributed to the increase in islet mass, we quantified total $\alpha$ and $\beta$ cell area separately. Surprisingly, while the CTR/HFD group displayed a near doubling of both $\beta$ cell and $\square$ cell area (Fig. 5.5a\&b), only $\beta$ cell area was significantly increased in the HFD/HFD (Fig. 5.5a). This leads to a normal $\beta / \alpha$ cell ratio in the CTR/HFD group but an increase in the $\beta / \alpha$ cell ratio in HFD/HFD animals (Fig. 5.5c). Since an increase in $\beta$ cell or $\alpha$ cell area could be a result of hyperplasia or hypertrophy, we also determined $\beta$ cell and $\alpha$ cell size and number. While $\beta$ cell size did not change in any of the groups (Fig. 5.6a), $\beta$ cell number was increased in both the CTR/HFD and HFD/HFD groups (Fig. 5.6b); however, the 
increase in the CTR/HFD was significantly greater than the HFD/HFD group. In contrast, $\alpha$ cell size was increased in both the CTR/HFD and HFD/HFD groups (Fig. 5.6c), even though only the CTR/HFD group displayed an increase in $\alpha$ cell number (Fig. 5.6d). This indicates that the increase in $\alpha$ cell area in the CTR/HFD and HFD/HFD groups is due to hyperplasia, while the increased $\beta$ cell area in the CTR/HFD group is due to hypertrophy.

Differential Islet Gene Expression of HFD Offspring. In order to determine which factors may be responsible for the increase in insulin secretion observed in HFD offspring, we analyzed the mRNA expression in the whole pancreas of various genes involved in insulin production and secretion. It should be noted that we have presented data as both gene expression normalized to a house-keeping gene ( $\beta$-actin (Fig. 5.78f) as well as normalized to $\beta$ cell area, $\alpha$ cell area, or islet area, depending on the cell types in which the gene of interest is expressed. This methodology has been previously described when whole-pancreas mRNA is used (Gatford, et al., 2008). Absolute insulin mRNA expression was significantly elevated only in HFD/HFD pancreas, when compared to the CTR/CTR group (Fig. 5.87a). When insulin mRNA levels were normalized to the $\beta$ cell area (insulin-producing cells), the effect in the HFD/HFD group was lost, but there was a significant overall maternal diet effect (Fig. 5.8a). This would indicate that the increase in the HFD groups may be partially dependent on the increase in $\beta$ cell area, but that maternal HFD diet causes an increase in cellular expression. Consistent with the lack of differences in circulating glucagon levels, glucagon mRNA was not significantly different between the groups (Fig. 5.7e), even after normalizing to the $\alpha$ cell area (Fig. 5.8e). The two key genes involved in the initiation of GSIS are the glucose transporter 
Glut2 that facilitates diffusion of glucose into the cell, and glucokinase (GK), the ratelimiting enzyme in glycolysis (Iynedjian, 1993; Matschinsky, 1996; Newgard \& McGarry, 1995). In preliminarily studies using immunohistochemistry, we demonstrated that the primary glucose transporter in Japanese macaque $\beta$ cells is Glut2 (Fig. 5.9). Interestingly, Glut2 expression (Fig. 5.7c) was significantly increased specifically in the CTR/HFD, while $G K$ expression was specifically elevated in HFD/HFD group (Fig. 5.7b). However, when normalized for $\beta$ cell area, the differences in Glut2 mRNA were completely eliminated, suggesting that the change in Glut 2 is dependent on changes in $\beta$ cell area. In contrast, when $G K$ expression was normalized to islet area, the effect in the HFD/HFD group was lost, but a decrease in expression in the CTR/HFD group was unmasked. This indicates that cellular expression of GK in the islet is normal in HFD/HFD animals, but lower in the CTR/HFD group.

Juveniles on the HFD are exposed to 4 times more fatty acids than the CTR animals (Grant, et al., 2011), which can augment insulin secretion through activation of the G-protein coupled receptor GPR40/FFAR1 (free fatty acid receptor 1) (Briscoe et al., 2003; Nolan, Madiraju, Delghingaro-Augusto, Peyot, \& Prentki, 2006). Offspring on the post-weaning HFD had significantly increased FFAR1 gene expression (Fig. 5.7d). Since FFAR1 has been confirmed in $\alpha$ cells as well as $\beta$ cells (Del Guerra et al., 2010; Flodgren et al., 2007; Tomita et al., 2006), FFAR1 expression was normalized to islet area. There was no significant effect on normalized FFARI expression (Fig. 5.8d), indicating that the up-regulation was likely secondary to the increase in islet mass, not individual cell gene expression. 


\section{Discussion}

It is clear from these studies that, in young animals, exposure to a HFD during the postnatal period can have a similar impact on overall weight gain and glucose homeostasis, irrespective of whether the animal was born to a mother on a CTR or HFD. However, these studies point to some fundamental differences in the underlying mechanisms leading to the impaired endocrine pancreas function between CTR and HFD offspring. First, it should be noted that HFD/HFD and CTR/HFD offspring displayed a higher average body weight, which was only significant in the HFD/HFD group, although the increase is only approximately $10 \%$. Furthermore, while these animals have increased adiposity, overall the animals are very lean, with less than $3 \%$ body fat. In spite of this modest phenotype, the HFD/HFD offspring have significantly increased fasting and GSIS. There is also a modest sex difference in the metabolic effects. Body weight in females is not significantly elevated, but CTR/HFD and HFD/HFD are insulin resistant at this stage. However, males are heavier and the effect of the HFD is more evident in this sex. Glucose levels are significantly elevated in both the HFD/CTR and the HFD/HFD males, indicating that the metabolic effect of maternal HFD is more robust in these animals. The sex effect that we have demonstrated is consistent with the findings in rodent models in which males tend to be more susceptible to fetal HFD induced metabolic effects (Dunn \& Bale, 2009; Estrany, Proenza, Llado, \& Gianotti, 2011; Gomez-Perez, Gianotti, Llado, \& Proenza, 2011; Medrikova et al., 2011). Importantly, these effects were independent of maternal obesity or insulin resistance.

Comstock, 100 
Islet mass is similarly increased in both the CTR/HFD and HFD/HFD offspring. However, the underlying factors that contribute to this increase are different. Animals exposed to the maternal HFD have larger islets, while the post-weaning HFD alone leads to an increased number of islets. Both effects result in increased islet mass, although morphological differences are likely to have differential long-term outcomes in these animals. By increasing small islet density, CTR/HFD animals may be able to resist the effect of the HFD for longer. Since islet maturation in the macaque continues through puberty, the smaller islets will be able to continue to expand in response to metabolic pressure and allow for increased insulin secretion in response to chronic, long-term HFD exposure. In contrast, larger islets indicate that the HFD/HFD islets have already started expanding in response to chronic HFD. The HFD/HFD juveniles will likely progress through the stages of insulin resistance leading to diabetes more rapidly by reaching the threshold of islet expansion sooner than the CTR/HFD juveniles.

We demonstrated that the CTR/HFD animals have increased $\alpha$ cell and $\beta$ cell mass, while the HFD/HFD offspring only have increased $\beta$ cell mass. In contrast, the HFD/CTR $\alpha$ cell and $\beta$ cell mass are normalized to control levels, indicating that islet cells are highly plastic during the peri-weaning period. We have previously demonstrated that fetuses from HFD mothers have decreased $\alpha$ cell mass. Therefore, the lack of $\alpha$ cell expansion in the HFD/HFD juveniles indicates that there is likely a programmed suppression of $\alpha$ cells that is a result of chronic HFD throughout the peri-natal period. This results in nearly a $40 \%$ increase in the $\beta / \alpha$ cell ratio in HFD/HFD offspring. While the CTR/HFD juveniles do have larger islets, the ratio of $\beta$ cells to $\alpha$ cells is maintained 
at normal levels, thus expectedly maintaining paracrine function of these islets. Intact paracrine regulation of islet cells is essential for long-term glucose homeostasis (Cabrera, et al., 2006; Walker, et al., 2011), indicating that the relative contribution of $\alpha$ cell and $\beta$ cells to islet composition will ultimately predict islet efficiency. It is not known whether the increase in $\beta / \alpha$ cell ratio in HFD/HFD juveniles is adaptive or maladaptive at this stage. Therefore, future studies in these offspring should be performed in order to determine the long-term effect that the differential ratio will have on glucose homeostasis.

We also established that the increase in $\beta$ cell mass in both CTR/HFD and HFD/HFD offspring is due to $\beta$ cell hyperplasia. Since there were no measurable differences in fetal $\beta$ cell mass from HFD mothers, this increase is likely due to the postnatal diet. This effect is similar to a rodent model in which post-natal HFD exposure leads to increased $\beta$ cell mass (Matveyenko, Singh, Shin, Georgia, \& Devaskar, 2010). However, the effects of maternal versus post-weaning HFD exposure on $\alpha$ cell development are novel when compared to other model species. In rodents and sheep models, $\alpha$ cell area tends to be elevated or unaffected by maternal HFD consumption (Cerf, et al., 2005; S. P. Ford, et al., 2009). The HFD/HFD juveniles have increased $\alpha$ cell size, but display decreased $\alpha$ cell number, suggesting that they are unable to yield more $\alpha$ cells in response to chronic HFD pressure. In contrast, the CTR/HFD offspring retain $\alpha$ cell plasticity demonstrably by increasing cell density when hypertrophy is not sufficient to balance augmented $\beta$ cell mass. These differences indicate that maternal HFD 
exposure limits the ability of $\alpha$ cells to replicate in response chronic post-natal HFD pressure.

Ultimately, both CTR/HFD and HFD/HFD juveniles are hyperinsulinemic, but analysis of gene expression in the pancreas provides evidence that the mechanism underlying the elevation in insulin is maternally programmed. However, it should be noted that total gene expression of the pancreas may be biased by the islet cell mass or density when islets are isolated from whole pancreas tissue. Accordingly, gene expression from isolated islets is biased toward large islets, because isolation protocols generally score islets as high-quality if they have a minimum specified diameter above at least $100 \mu \mathrm{M}$ (Matsumoto et al., 2002). Therefore, in order to obtain gene expression that is representative of islet content, we normalized gene expression to the area of tissue in which the gene is expressed (i.e., $\alpha$ cell area, $\beta$ cell area, or islet area). After normalization, the key differences demonstrated were an up-regulation of insulin expression, which is programmed by the maternal HFD and a down-regulation in GK in the CTR/HFD juveniles. Previous studies have shown that GK is the rate-limiting step in the initial phases of glucose-stimulated insulin secretion (De Vos et al., 1995; Liang, Najafi, \& Matschinsky, 1990). Decreased GK expression in the CTR/HFD animals will likely cause these islets to be less responsive to glucose, while the $\beta$ cells of HFD/HFD juveniles are primed to respond to glucose. It is unclear what causes the increased secretion demonstrated in CTR/HFD offspring. FFAR1 expression analysis indicates that expression of this receptor rises with increasing islet area. Fatty acid potentiation of glucose-stimulated insulin secretion is an additional factor that may contribute to the 
increased insulin secretion measured in both post-weaned HFD juvenile groups. However, future in-vivo and gene-expression studies are essential to determine the role both glucose and FFAs play in modulation of GSIS in all diet groups.

Upon review of the effects of HFD in these animals, it is evident that HFD consumption during pregnancy leads to decreased a cell plasticity in the juvenile Japanese macaque. When animals are returned to a control diet, most factors are ameliorated, indicating that this is a dynamic period of islet development. However, maternally programmed metabolic effects are still evident to some extent in these animals, since not all factors are normalized to control levels. Post-natal HFD exposure appears to exacerbate the effects of early HFD programming. $\beta$ cells are hyper-responsive in the HFD/HFD juveniles (fasting insulin levels are doubled, but $\beta$ cell mass is only increased by $30 \%$ ). This hyper-responsiveness is indicative of early insulin resistance and the ultimate progression toward diabetes (Ahlgren, et al., 1998; Weir \& BonnerWeir, 2004; Weir et al., 2009).

While there is a rise in $\beta$ cell to $\alpha$ cell ratio in HFD/HFD juveniles, these animals are still apparently capable of managing the metabolic insult. In HFD/HFD animals, $\alpha$ cells hypersecrete glucagon. It is likely that by suppressing $\alpha$ cell proliferation, glucagon levels are maintained at control levels. However, long-term exposure to the HFD could increase the susceptibility of HFD/HFD animals to diabetes because they will be unable to maintain an appropriate islet paracrine dynamic, which is essential for normal glucose homeostasis. It is unclear if this imbalance in the islet cell ratio prepares the animal to combat future metabolic pressure, or if they will reach a peak at which islets are no 
longer able to withstand the metabolic insult. We anticipate that, by following the HFD/HFD through the adolescent period, these animals will ultimately reach the plateau of adaptation and start to decline into early metabolic syndrome. Eventually the CTR/HFD and HFD/HFD are likely to develop similar metabolic complications because adaptation to this HFD is unsustainable. However, these studies suggest that the mechanisms in which the disease progresses and ultimately the timing of disease progression will be accelerated in the HFD/HFD animals. This is similar to the human population in which mothers consuming a HFD give rise to children that succumb to metabolic pressures earlier in life. 
Chapter 5 Figures

Comstock, 106 
Figure 5.1: Juvenile Physiology
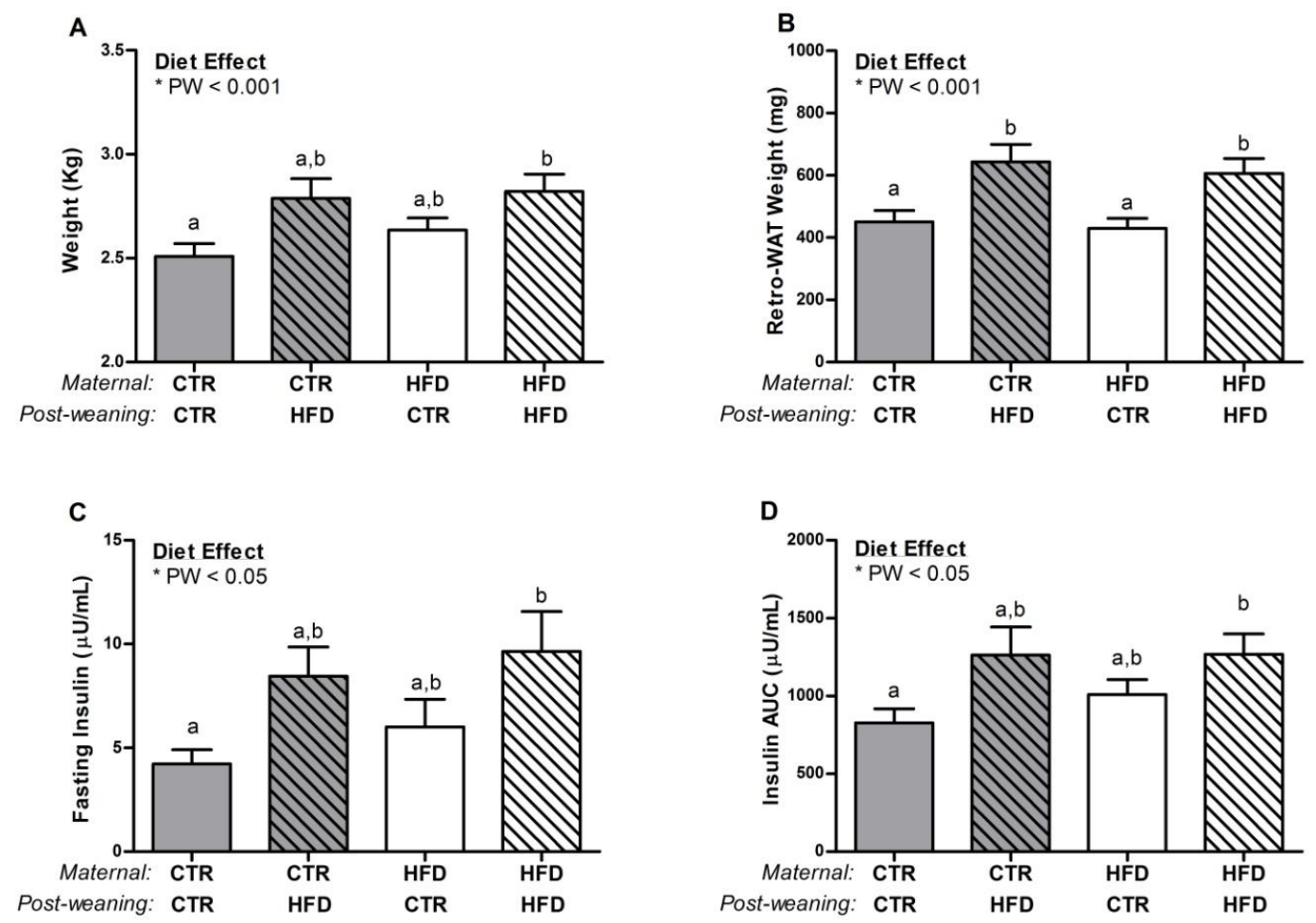

HFD/HFD animals were significantly heavier than the CTR/CTR, while CTR/HFD displayed a similar trend (A) and RWAT was significantly elevated in the CTR/HFD and HFD/HFD juveniles (B). HFD/HFD fasting (C) insulin and insulin area under the curve (D) were significantly elevated when compared to CTR/CTR. Weight, RWAT weight, fasting insulin and insulin AUC were significantly affected by the post-weaning diet. Two-way ANOVA with Bonferroni's post-hoc analysis. CTR/CTR, $\mathrm{n}=22$; CTR/HFD, $\mathrm{n}=9$; HFD/CTR, $\mathrm{n}=14 ;$ HFD/HFD, $\mathrm{n}=21$. 
Figure 5.2: Sex Differences in Body Weight and Fasting Glucose
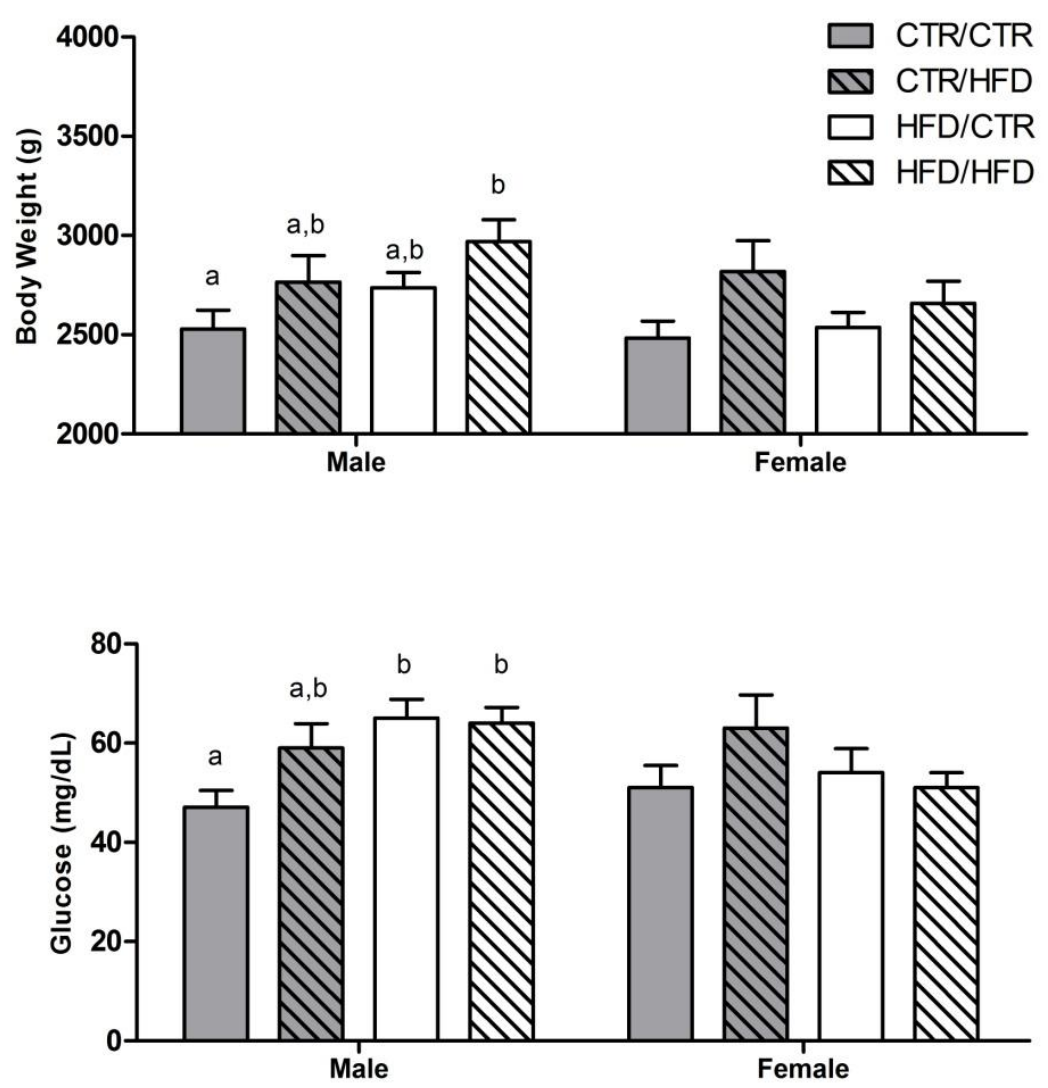

HFD/HFD male offspring have significantly increased body weight, while no significant difference was demonstrated in the females (A). HFD/CTR and HFD/HFD have increased fasting glucose secretion and female groups are not significantly different $(\mathbf{B})$. Two-way ANOVA with Bonferroni's post-hoc analysis. Males: CTR/CTR, $\mathrm{n}=12$; CTR/HFD, n=5; HFD/CTR, n=7; HFD/HFD, n=11. Females: : CTR/CTR, n = 10; CTR/HFD, n=4; HFD/CTR, n=7; HFD/HFD, n=10. 
Figure 5.3: Juvenile Islet Mass and Contributing Factors
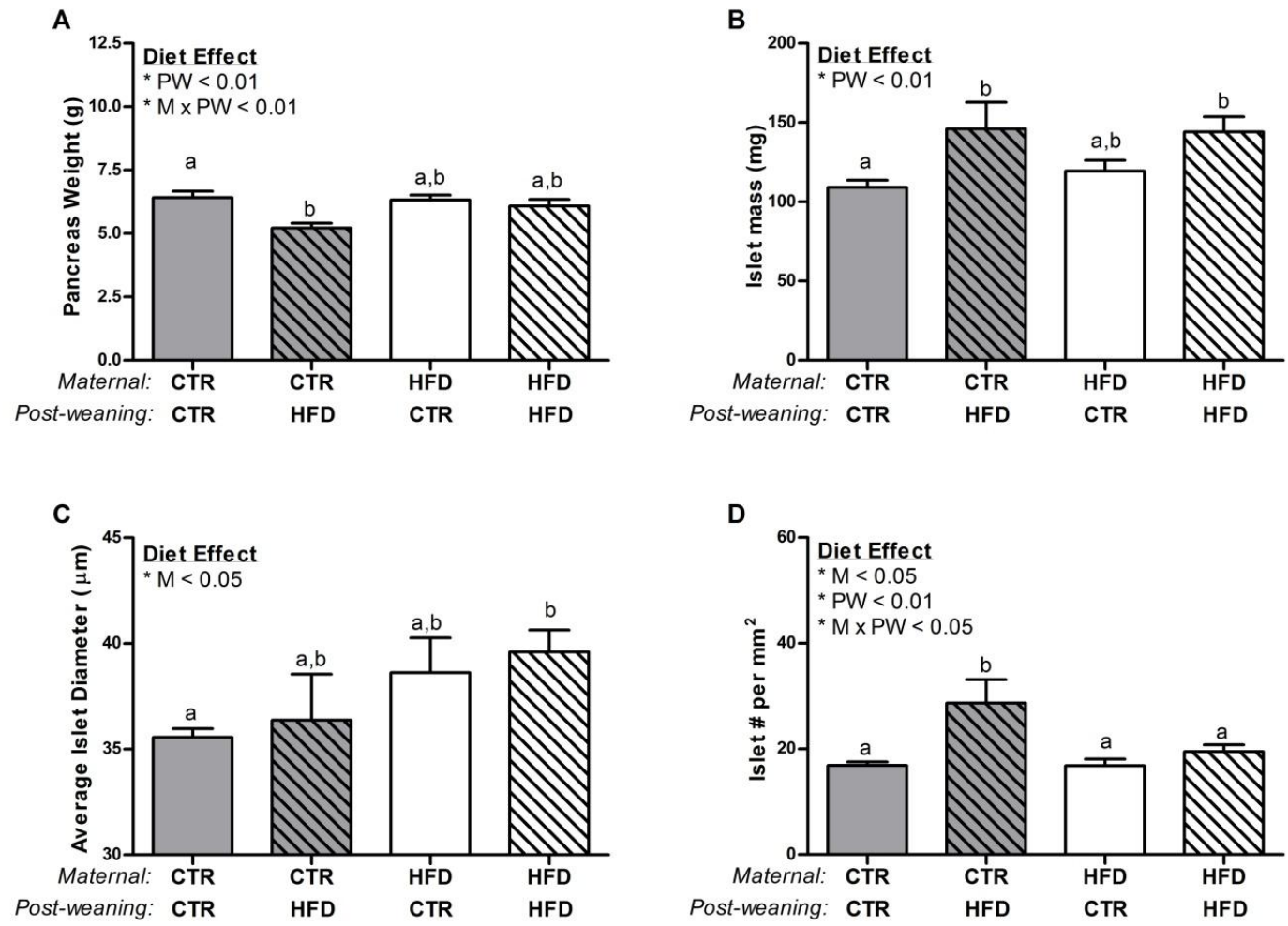

Pancreas weight was decreased in the CTR/HFD group due to a post-weaning and an interacting diet effect (A). Both CTR/HFD and HFD/HFD juveniles had a 30\% increase in islet mass as compared to CTR/CTR animals, which is significantly affected by the post-weaning diet (B). Mean islet diameter was significantly elevated only in the HFD/HFD offspring, due to a maternal diet effect (C) while islet density was only significantly elevated in CTR/HFD and displayed a maternal, post-weaning and interacting diet effect (D). Two-way ANOVA with Bonferroni's post-hoc analysis. CTR/CTR, $\mathrm{n}=7$, CTR/HFD, $\mathrm{n}=6$; HFD/CTR, n=7; HFD/HFD, n=7. 
Figure 5.4: Relative Mass, Diameter and Density of Islets

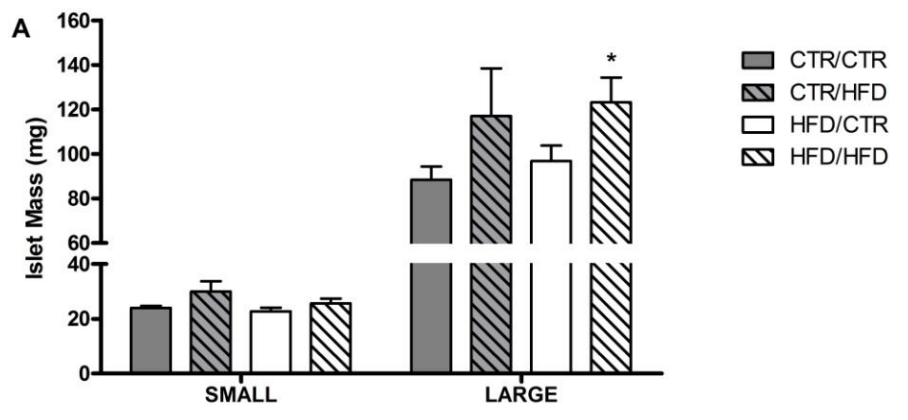

B
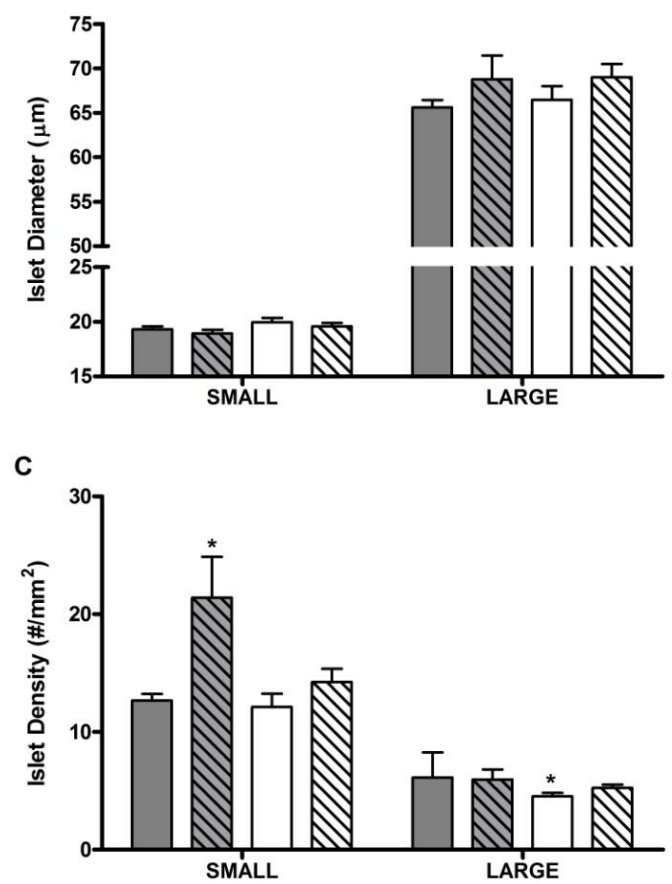

Large islet mass was significantly increased in the HFD/HFD as compared to CTR/CTR (A). Islet diameter was not significantly affected when islets were separated by size (B). Small islet density was significantly increased in the CTR/HFD juveniles and large islet density was decreased in the HFD/CTR juveniles as compared to CTR/CTR (C). *p < 0.05 CTR/CTR, 2-way anova with post-hoc analysis; $\mathrm{n}=7$, CTR/HFD, $\mathrm{n}=6$; HFD/CTR, $\mathrm{n}=7$; HFD/HFD, $\mathrm{n}=7$. 
Figure 5.5: $\alpha$ cell and $\beta$ cell Mass
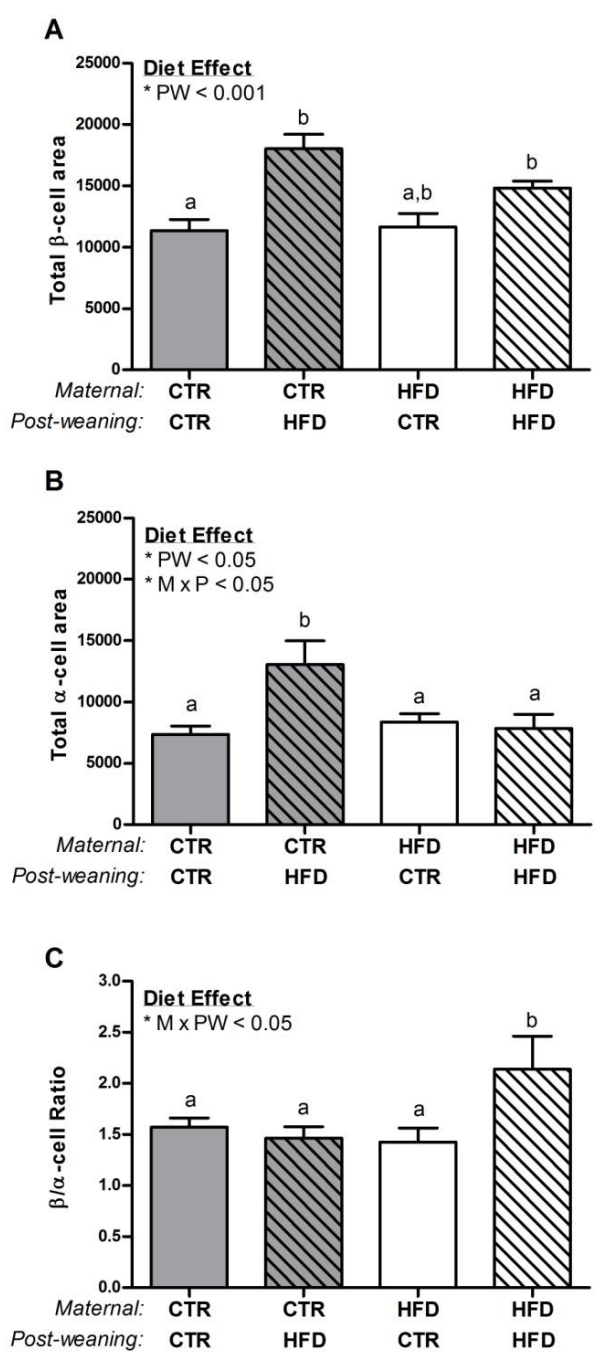

$\beta$ cell area was increased in both the CTR/HFD the HFD/HFD juveniles, demonstrating a post-weaning diet effect (A). Only the CTR/HFD juveniles displayed significantly increased $\alpha$ cell area due to a post-weaning and interacting diet effect (B). HFD/HFD offspring displayed nearly a $50 \%$ increase in $\beta / \alpha$ cell ratio due to an interaction between the maternal and post-weaning diet. Two-way ANOVA with Bonferroni's post-hoc analysis. CTR/CTR, $n=7$, CTR/HFD, $n=6$; HFD/CTR, n=7; HFD/HFD, $n=7$. 
Figure 5.6: $\beta$ cell and $\alpha$ cell Quantification
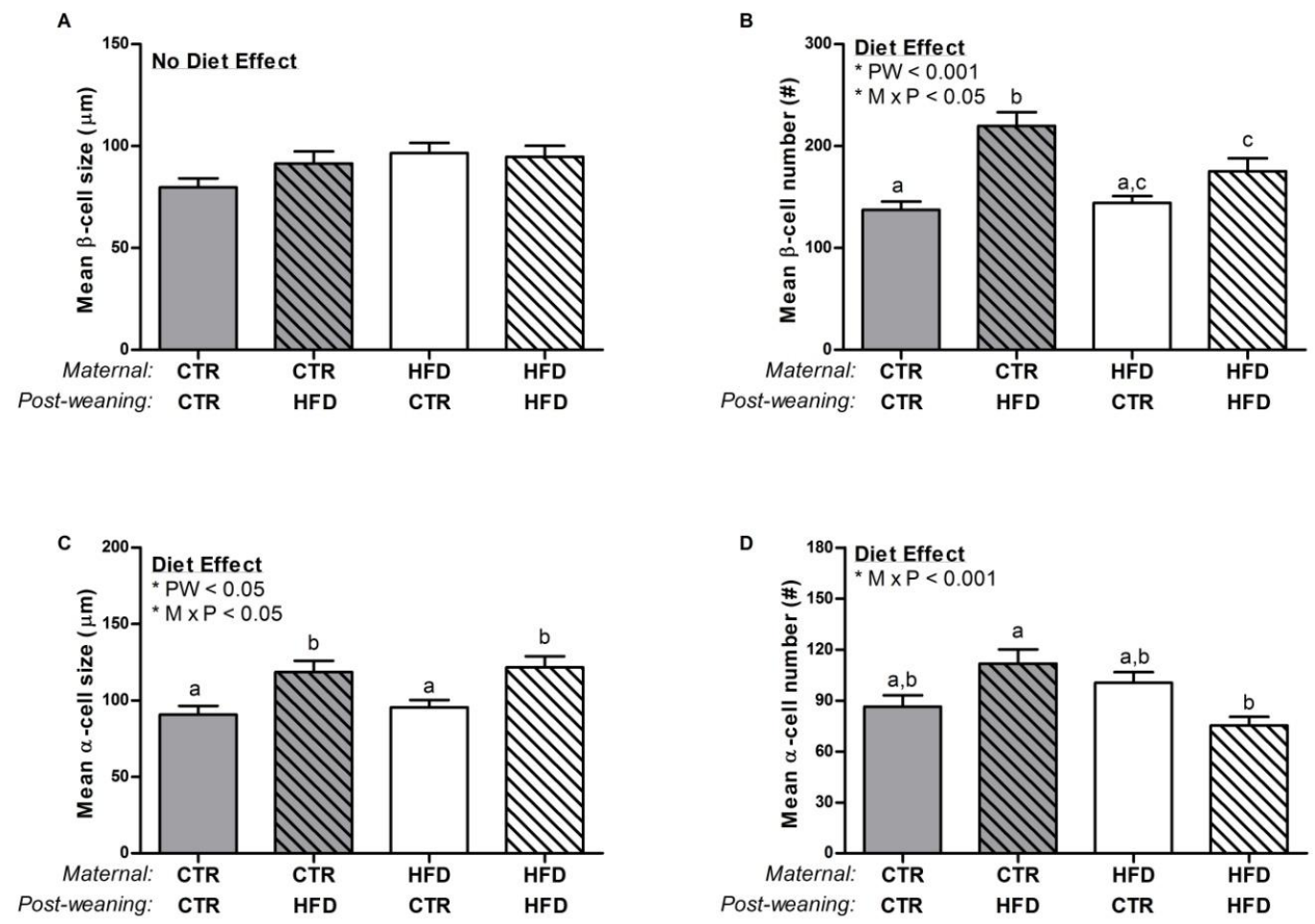

There was no change in size of $\beta$ cells from all juvenile groups (A) while $\beta$ cells from both HFD groups displayed significant hyperplasia and had a significant post-weaning and interacting diet effect (B). $\alpha$ cell hypertrophy was significant in both CTR/HFD and HFD/HFD offspring due to a post-weaning and interacting diet effect (C). Finally, while CTR/HFD $\alpha$ cells tended to be hyperplasic, HFD/HFD displayed a decreased number compared to the CTR/HFD animals. This response is due to a interacting diet effect (D). Two-way ANOVA with Bonferroni's post-hoc analysis. CTR/CTR $n=30$, CTR/HFD $n$ $=35, \mathrm{HFD} / \mathrm{CTR} \mathrm{n}=30, \mathrm{HFD} / \mathrm{HFD} \mathrm{n}=25$. 
Figure 5.7: Gene Expression in Juvenile Pancreas
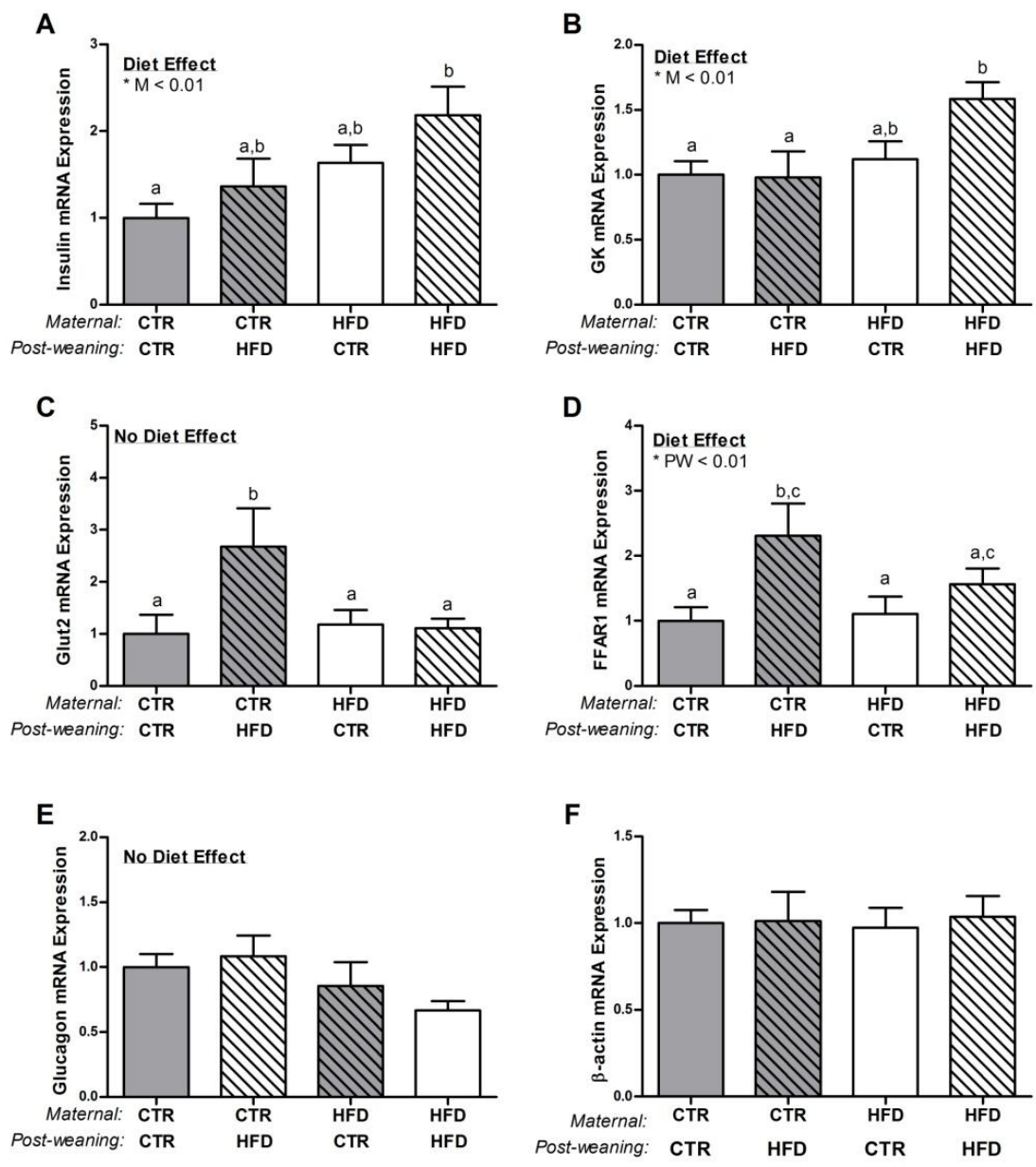

Insulin (A) and glucokinase (B) mRNA expression were significantly elevated in the HFD/HFD group as compared to the CTR/CTR juveniles. These genes were significantly affected by the maternal diet. The facilitated glucose transporter, Glut2 displayed no diet effect, but was significantly elevated in the CTR/HFD animals $(\mathbf{C})$. The $\beta$ cell free fatty acid receptor FFAR1 (D) was also elevated in the CTR/HFD group and displayed a postweaning diet effect. Glucagon was not significantly different between groups (E). All genes were normalized to $\beta$-actin $(\mathbf{F})$. Two-way ANOVA with Bonferroni's post-hoc analysis. CTR/CTR, $\mathrm{n}=12$; CTR/HFD, $\mathrm{n}=6$; HFD/CTR, $\mathrm{n}=9$; HFD/HFD, $\mathrm{n}=12$. 
Figure 5.8: Normalized Gene expression in Juvenile Pancreas
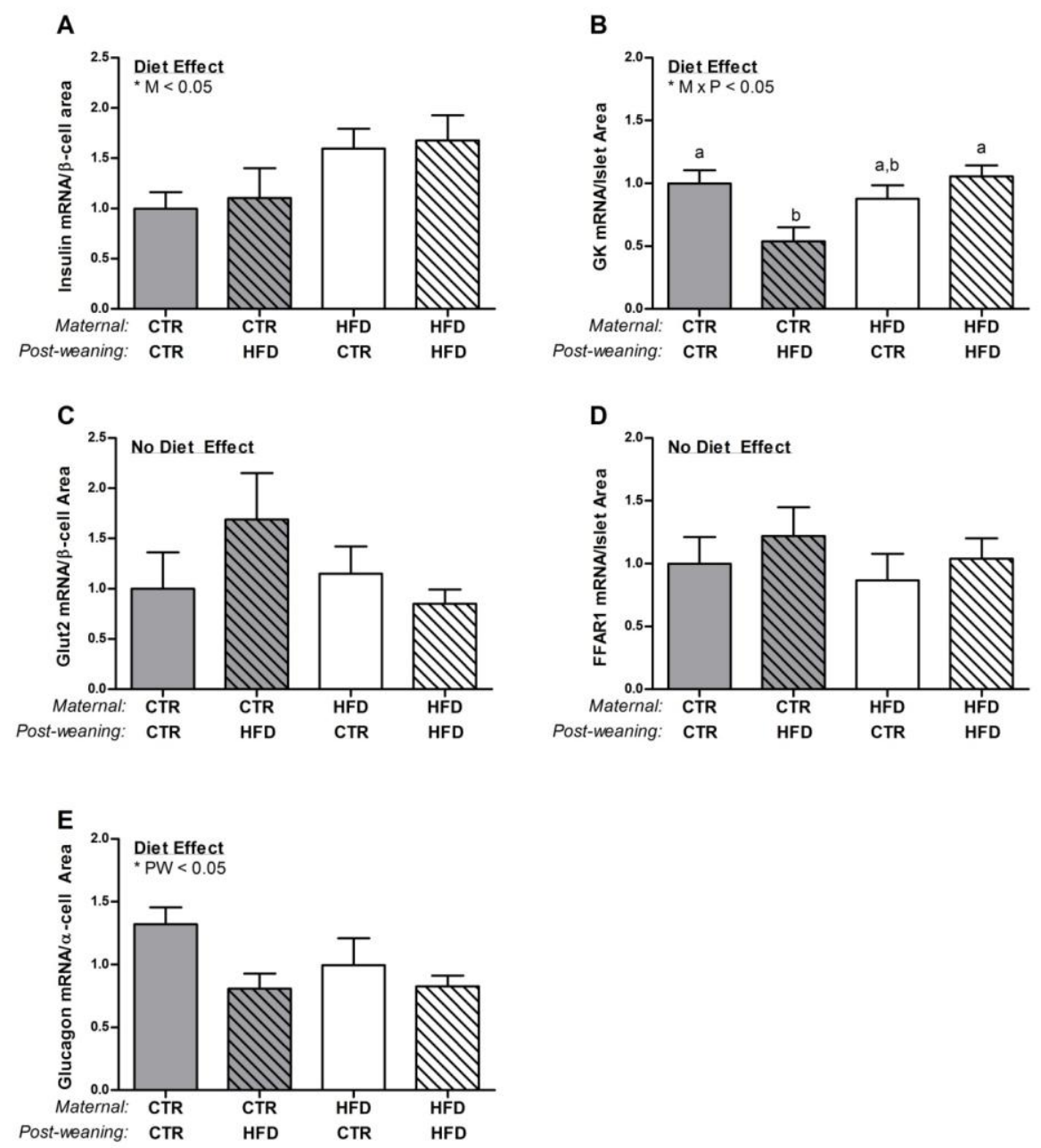

Insulin mRNA/ $\beta$ cell area is significantly affected by the maternal diet, but not different between juvenile groups (A). Glucokinase mRNA/Islet Area is significantly downregulated in the CTR/HFD animals and affected by an interaction between the maternal and post-weaning diet (B). Glut $2 / \beta$ cell area is not significantly affected (C). FFAR1 mRNA/Islet Area is not significantly affected (D). Glucagon mRNA/ $\alpha$ cell area is affected by the post-weaning diet but not significantly different between groups (E). All genes were also normalized to $\beta$-actin. Two-way ANOVA with Bonferroni's post-hoc analysis. CTR/CTR, $n=12$; CTR/HFD, n=6; HFD/CTR, n=9; HFD/HFD, n=12. 
Figure 5.9: Glut2 versus Glut1 Expression
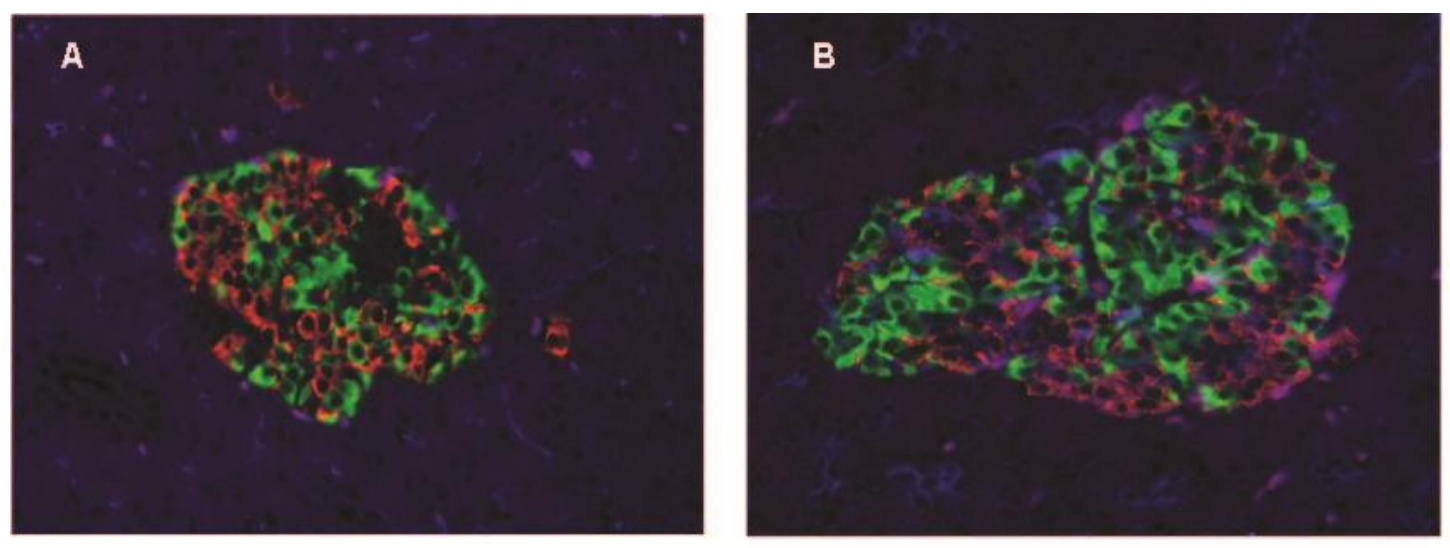

C Glut1 vs Glut2: Normalized to ALG9

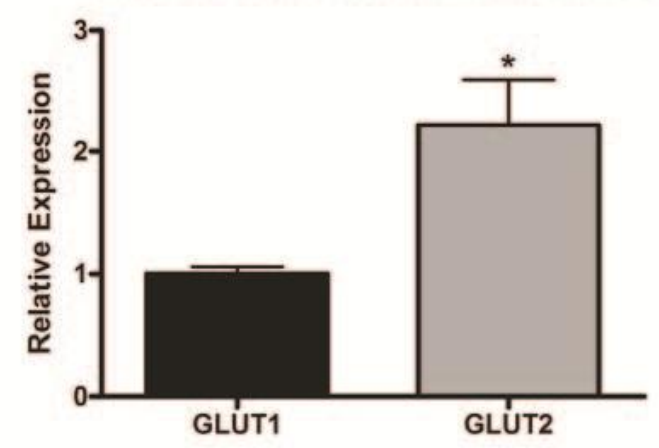

IHC of pancreas sections demonstrate that Glut1 is limited to exocrine tissue (A) while Glut2 is present in islets, principally $\beta$ cells (B). GLUT1 or GLUT2 labeled in blue, insulin labeled in red, glucagon in green and co-labeled insulin and GLUT2 is purple. Glut2 m RNA expression is more than double the expression of Glut1 mRNA in pancreas tissue, Student's t-test, $\mathrm{n}=39,{ }^{*} \mathrm{p}<0.05(\mathbf{C})$. 


\section{Chapter 5 Tables}

Comstock, 116 
Table 5.1: Juvenile Physiology

\begin{tabular}{|c|c|c|c|c|c|}
\hline Juvenile Physiology & CTR/CTR & CTR/HFD & HFD/CTR & HFD/HFD & Effect \\
\hline$n$ & 22 & 9 & 14 & 21 & \\
\hline Weight (g) & $2508 \pm 63^{a}$ & $2788 \pm 96^{a, b}$ & $2636 \pm 59^{a, b}$ & $2820 \pm 84^{b}$ & PW, S \\
\hline Pancreas weight (g) & $6.43 \pm 0.25^{a}$ & $5.23 \pm 0.19^{b}$ & $6.33 \pm 0.19^{a, b}$ & $6.08 \pm 0.26^{a, b}$ & - \\
\hline Pancreas/Body Weight & $2.67 \pm 0.06^{a}$ & $1.97 \pm 0.07^{b}$ & $2.40 \pm 0.05^{a}$ & $2.18 \pm 0.07^{c}$ & PW \\
\hline Total Body Fat $\%$ & $1.81 \pm 0.22$ & $2.54 \pm 0.21$ & $2.16 \pm 0.36$ & $2.30 \pm 0.31$ & - \\
\hline Insulin $(\mu \mathrm{U} / \mathrm{mL})$ & $4.25 \pm 0.69^{a}$ & $8.45 \pm 1.41^{a, b}$ & $6.00 \pm 1.34^{a, b}$ & $9.64 \pm 1.93^{b}$ & PW \\
\hline Glucose (mg/dL) & $49 \pm 2.8$ & $61 \pm 3.7$ & $60 \pm 2.6$ & $56 \pm 1.6$ & $\mathrm{MxPW}, \mathrm{MxS}$ \\
\hline IVGTT - GSIS & $828 \pm 89^{a}$ & $1263 \pm 182^{a, b}$ & $1010 \pm 96^{a, b}$ & $1267 \pm 132^{b}$ & PW \\
\hline IVGTT - GAUC & $4789 \pm 253$ & $5406 \pm 344$ & $5196 \pm 293$ & $4601 \pm 215$ & - \\
\hline IVGTT - Total IAUC & $1184 \pm 113^{a}$ & $1655 \pm 275^{a, b}$ & $1398 \pm 134^{a, b}$ & $1690 \pm 103^{b}$ & PW \\
\hline 1st Phase Insulin & $39.2 \pm 6.2$ & $50.9 \pm 7.3$ & $41.6 \pm 8.6$ & $55.6 \pm 6.0$ & - \\
\hline 2nd Phase Insulin & $1055 \pm 102$ & $1469 \pm 259$ & $1255 \pm 113$ & $1496 \pm 92$ & PW \\
\hline HOMA-IR & $0.49 \pm 0.08^{a}$ & $1.28 \pm 0.23^{a, b}$ & $0.91 \pm 0.23^{a, b}$ & $1.48 \pm 0.33^{b}$ & PW \\
\hline Glucagon (pg/mL) & $135 \pm 12.1$ & $102.7 \pm 5.7$ & $131.0 \pm 18.7$ & $132.5 \pm 13.9$ & - \\
\hline Leptin (ng/mL) & $1.12 \pm 0.06$ & $1.23 \pm 0.21$ & $1.28 \pm 0.12$ & $1.07 \pm 0.10$ & - \\
\hline
\end{tabular}

Physiological measurements of 4 offspring groups. Three-way ANOVA with Bonferroni's post-hoc analysis. 
Table 5.2: Juvenile Female Physiology

\begin{tabular}{|l|c|c|c|c|c|}
\hline 13 Months Females & CTR/CTR & CTR/HFD & HFD/CTR & HFD/HFD & Effect \\
\hline $\mathrm{n}$ & 10 & 4 & 7 & 10 & \\
\hline Weight (g) & $2483 \pm 85$ & $2818 \pm 156$ & $2535 \pm 77$ & $2657 \pm 112$ & $\mathrm{PW}$ \\
\hline Pancreas weight (g) & $6.41 \pm 0.27$ & $5.23 \pm 0.35$ & $6.07 \pm 0.27$ & $5.69 \pm 0.34$ & $\mathrm{PW}$ \\
\hline Pancreas/Body Weight & $2.60 \pm 0.08^{\mathrm{a}}$ & $1.85 \pm 0.07^{\mathrm{b}}$ & $2.39 \pm 0.06^{\mathrm{a}}$ & $2.16 \pm 0.13^{\mathrm{c}}$ & $\mathrm{MXPW}, \mathrm{PW}$ \\
\hline Total Body Fat \% & $1.63 \pm 0.33$ & $2.44 \pm 0.44$ & $2.83 \pm 0.57$ & $2.39 \pm 0.43$ & - \\
\hline RWAT Weight $(\mathrm{mg})$ & $434 \pm 49$ & $680 \pm 64$ & $476 \pm 43$ & $610 \pm 92$ & $\mathrm{PW}$ \\
\hline Insulin $(\mu \mathrm{U} / \mathrm{mL})$ & $4.94 \pm 1.28$ & $8.80 \pm 1.33$ & $4.87 \pm 1.40$ & $9.50 \pm 3.56$ & - \\
\hline Glucose $(\mathrm{mg} / \mathrm{dL})$ & $51 \pm 4.5$ & $63 \pm 6.7$ & $54 \pm 4.9$ & $51 \pm 3.0$ & - \\
\hline IVGTT - GSIS & $789 \pm 128^{\mathrm{a}}$ & $1612 \pm 148^{\mathrm{b}}$ & $995 \pm 176^{\mathrm{a}, \mathrm{b}}$ & $1249 \pm 137^{\mathrm{a}, \mathrm{b}}$ & $\mathrm{MXPW,} \mathrm{M}$ \\
\hline IVGTT - GAUC & $4188 \pm 381$ & $5581 \pm 748$ & $4825 \pm 463$ & $4627 \pm 299$ & - \\
\hline IVGTT - Total IAUC & $1227 \pm 91$ & $1785 \pm 413$ & $1423 \pm 242$ & $1806 \pm 150$ & $\mathrm{PW}$ \\
\hline 1st Phase Insulin & $33.3 \pm 3.4$ & $55.4 \pm 11.6$ & $39.5 \pm 14.4$ & $47.8 \pm 5.8$ & - \\
\hline 2nd Phase Insulin & $1103 \pm 84$ & $1574 \pm 403$ & $1283 \pm 203$ & $1625 \pm 138$ & PW \\
\hline HOMA-IR & $0.62 \pm 0.15$ & $1.42 \pm 0.33$ & $0.65 \pm 0.29$ & $1.29 \pm 0.55$ & $\mathrm{PW}$ \\
\hline Glucagon (pg/mL) & $141.4 \pm 18.7$ & $105.2 \pm 8.9$ & $139.5 \pm 34.7$ & $150.8 \pm 24.3$ & - \\
\hline Leptin (ng/mL) & $1.20 \pm 0.10$ & $0.72 \pm 0.22$ & $1.30 \pm 0.14$ & $1.71 \pm 0.47$ & PW \\
\hline
\end{tabular}

Physiological measurements of females from offspring separately analyzed by sex. Twoway ANOVA with Bonferroni's post-hoc analysis. 
Table 5.3: Juvenile Male Physiology

\begin{tabular}{|c|c|c|c|c|c|}
\hline 13 Month Males & CTR/CTR & CTR/HFD & HFD/CTR & HFD/HFD & Effect \\
\hline $\mathrm{N}$ & 12 & 5 & 7 & 11 & \\
\hline Weight (g) & $2529 \pm 94^{a}$ & $2764 \pm 134^{a, b}$ & $2736 \pm 77^{a, b}$ & $2969 \pm 110^{b}$ & PW \\
\hline Pancreas weight (g) & $6.75 \pm 0.49$ & $5.70 \pm 0.50$ & $6.59 \pm 0.24$ & $6.40 \pm 0.38$ & - \\
\hline Pancreas/Body Weight & $2.74 \pm 0.08^{a}$ & $2.05 \pm 0.11^{b}$ & $2.41 \pm 0.09^{a}$ & $2.19 \pm 0.09^{b}$ & MXPW, PW \\
\hline Total Body Fat \% & $1.96 \pm 0.32$ & $2.64 \pm 0.07$ & $1.66 \pm 0.22$ & $2.15 \pm 0.60$ & - \\
\hline RWAT Weight (mg) & $463 \pm 54^{a}$ & $615 \pm 91^{a, b}$ & $390 \pm 45^{a}$ & $672 \pm 81^{b}$ & PW \\
\hline Insulin $(\mu \mathrm{U} / \mathrm{mL})$ & $3.56 \pm 0.64$ & $8.24 \pm 2.23$ & $7.23 \pm 2.06$ & $10.0 \pm 2.42$ & - \\
\hline Glucose (mg/dL) & $47 \pm 3.4^{a}$ & $59 \pm 4.9^{a, b}$ & $65 \pm 3.8^{b}$ & $64 \pm 3.1^{b}$ & $\mathrm{M}$ \\
\hline IVGTT - GSIS & $856 \pm 127^{a}$ & $1053 \pm 238^{a, b}$ & $1025 \pm 94^{a, b}$ & $1285 \pm 233^{b}$ & - \\
\hline IVGTT - GAUC & $5171 \pm 291$ & $5301 \pm 395$ & $5567 \pm 328$ & $4578 \pm 328$ & - \\
\hline IVGTT - Total IAUC & $1152 \pm 190$ & $1551 \pm 404$ & $1368 \pm 104$ & $1584 \pm 139$ & - \\
\hline 1st Phase Insulin & $43.6 \pm 10.7$ & $47.3 \pm 10.3$ & $44.1 \pm 9.4$ & $50.4 \pm 5.4$ & - \\
\hline 2nd Phase Insulin & $1019 \pm 170$ & $1384 \pm 375$ & $1222 \pm 90$ & $1380 \pm 119$ & - \\
\hline HOMA-IR & $0.38 \pm 0.05^{a}$ & $1.19 \pm 0.34^{a, b}$ & $1.14 \pm 0.34^{a, b}$ & $1.65 \pm 0.40^{b}$ & $\mathrm{PW}$ \\
\hline Glucagon $(\mathrm{pg} / \mathrm{mL})$ & $129.7 \pm 16.4$ & $101.2 \pm 8.1$ & $124.9 \pm 22.6$ & $115.9 \pm 14.0$ & - \\
\hline Leptin (ng/mL) & $1.05 \pm 0.07^{a, b}$ & $1.53 \pm 0.21^{b}$ & $1.26 \pm 0.21^{a, b}$ & $0.91 \pm 0.06^{a}$ & MXPW \\
\hline
\end{tabular}

Physiological measurements of males from offspring groups separately analyzed by sex Two-way ANOVA with Bonferroni's post-hoc analysis. 
Table 5.4: Real-time PCR Values of Juvenile Pancreas

\begin{tabular}{|c|c|c|c|c|c|}
\hline Real-time QPCR & CTR/CTR & CTR/HFD & HFD/CTR & HFD/HFD & Effect \\
\hline $\mathrm{n}$ & 7 & 6 & 6 & 6 & \\
\hline ALG9 & $1.00 \pm 0.06$ & $1.01 \pm 0.07$ & $1.02 \pm 0.10$ & $0.96 \pm 0.03$ & - \\
\hline FFAR1 (GPR40) & $1.00 \pm 0.21^{a}$ & $2.31 \pm 0.50^{b, c}$ & $1.11 \pm 0.27^{\mathrm{a}}$ & $1.56 \pm 0.24^{a, c}$ & PW \\
\hline Glucagon (GCG) & $1.00 \pm 0.10$ & $1.08 \pm 0.16$ & $0.85 \pm 0.18$ & $0.67 \pm 0.07$ & - \\
\hline Glucokinase (GK) & $1.00 \pm 0.10^{a}$ & $0.98 \pm 0.20^{a}$ & $1.22 \pm 0.14^{a, b}$ & $1.58 \pm 0.13^{b}$ & $M$ \\
\hline Glut1 & $1.00 \pm 0.05$ & $0.98 \pm 0.02$ & $1.07 \pm 0.11$ & $1.11 \pm 0.13$ & - \\
\hline Glut2 & $1.00 \pm 0.36^{\mathrm{a}}$ & $2.67 \pm 0.74^{b}$ & $1.28 \pm 0.28^{a}$ & $1.11 \pm 0.18^{a}$ & - \\
\hline Insulin & $1.00 \pm 0.16^{a}$ & $1.36 \pm 032^{a, b}$ & $1.63 \pm 0.21^{a, b}$ & $2.18 \pm 0.33^{b}$ & $\mathrm{M}$ \\
\hline$P D X 1$ & $1.00 \pm 0.11$ & $1.20 \pm 0.07$ & $0.99 \pm 0.09$ & $0.95 \pm 0.07$ & - \\
\hline
\end{tabular}

Real-time PCR expression of genes in juvenile pancreas. Two-way ANOVA with Bonferroni's post-hoc analysis. CTR/CTR, $\mathrm{n}=7$; CTR/HFD, $\mathrm{n}=6$; HFD/CTR, $\mathrm{n}=6$; HFD/HFD, $n=6$. 
Table 5.5: $\alpha$ cell and $\beta$ cell Measurements

\begin{tabular}{|l|c|c|c|c|}
\hline Measurements & CTR/CTR & CTR/HFD & HFD/CTR & HFD/HFD \\
\hline Average $\alpha$ cell size $\left(\mu \mathrm{m}^{2}\right)$ & $90.83 \pm 5.61$ & $118.5 \pm 7.42 *$ & $95.61 \pm 4.65$ & $121.6 \pm 7.43 *$ \\
\hline Average $\alpha$ cell number & $86.35 \pm 6.71$ & $111.7 \pm 8.34 *$ & $100.4 \pm 6.33$ & $75.25 \pm 5.16+$ \\
\hline Average $\alpha$ cell area $\left(\mu \mathrm{m}^{2}\right)$ & 7237 & 13098 & 8834 & 8240 \\
\hline Average $\beta$ cell size $\left(\mu \mathrm{m}^{2}\right)$ & $79.8 \pm 4.32$ & $91.4 \pm 6.07$ & $96.6 \pm 4.94$ & $94.7 \pm 5.48$ \\
\hline Average $\beta$ cell number & $137.4 \pm 8.10$ & $219.9 \pm 13.4 *$ & $144.1 \pm 6.91$ & $175.4 \pm 12.9 *$ \\
\hline Average $\beta$ cell area $\left(\mu \mathrm{m}^{2}\right)$ & 10458 & 18357 & 13524 & 16019 \\
\hline$\beta / \alpha$ cell Area & 1.45 & 1.4 & 1.53 & 1.94 \\
\hline$\%$ Ratio & $100.00 \%$ & $96.99 \%$ & $105.94 \%$ & $134.53 \%$ \\
\hline
\end{tabular}

Analysis of $\alpha$ and $\beta$ cell area, size, number and relative $\beta / \alpha$ cell ratio of the 4 groups. Islets stereologically randomly selected for each animal. CTR/CTR $n=30$, CTR/HFD $n$ $=35$, HFD $/$ CTR $n=30$, HFD/HFD $n=25$. 
Chapter 6: Limitations and Future Studies

Comstock, 122 


\section{Limitations of the HFD Fed Macaque Model}

While the macaque is an excellent animal model to study early MetS and disease progression to T2DM, it does have some limitations. First, it should be noted that the cost of this model compared to rodent and sheep models is high, which causes experimental limits on these studies based on funding. However, since these animals are so similar to humans in the development of MetS and T2DM, whenever possible it is essential to design experiments that could be specifically tested in this model. Rodent studies make an excellent starting point. However, much of the development of the pancreas and other organs impacted by HFD consumption in the rodent occurs during the early post-natal period, whereas the monkey and human have similar fetal development (Aguayo-Mazzucato, Sanchez-Soto, Godinez-Puig, Gutierrez-Ospina, \& Hiriart, 2006; Fowden \& Hill, 2001; Remacle, et al., 2007; Scaglia, et al., 1997). The sheep has also been extensively used in developmental work; however, although these animals have similar development to humans, they have ruminant digestion and do not process fats (Oltjen \& Beckett, 1996; Sanz Sampelayo, Chilliard, Schmidely, \& Boza, 2007). Therefore, the effects of maternal obesity induced by increased caloric intake can be studied in fetal offspring, but it is not possible to study the effect of HFD in these animals. Furthermore, they have a cotyledonary placenta that is very different from the human and macaque placenta

A second limitation of this model is the time points of animal sacrifice and tissue collection that we selected. In many rodent models, animals are sacrificed sequentially throughout HFD exposure at intervals of a few days to a few weeks (Cerf, et al., 2005; Scaglia, et al., 1997). This allows the investigator to understand sequential development 
of the conditions that lead to the phenotype studied. We limited collection time points to the early third trimester and the postnatal 13-month time point, due to limited animal availability, cost of the animals, and ethical considerations - sacrificing as few animals as possible to gain the most knowledge. Therefore, reasonable deductions about islet development were made based on the morphology of islets at the two time points and the physiological presentation of the animal between those time points. However, the time points that we chose to characterize are critical for islet development. As a point of emphasis, it has taken approximately ten years, to produce sufficient offspring to perform these studies. This leads to a third limitation of the model, which is small sample size. Sample size within these groups is somewhat limited ( $\mathrm{n}=9$ to 21/group), when compared to many rodent studies. However, compared to other primate studies (Choi et al., 2011; de Koning, Bodkin, Hansen, \& Clark, 1993), the sample size is relatively robust and it has sufficient power to determine significant differences between groups. In spite of this limitation our studies as a whole have identified key insights into the developmental abnormalities that may contribute to early childhood obesity. In turn, we have increased the awareness of the complications that may occur in mothers and their offspring who are obese or consume a HFD during the pregnancy period. The experiments performed in these animals have already begun to enlighten clinical investigators about the critical period of development in fetal life and can help them understand how to intervene when necessary. In addition, by understanding the effects of HFD consumption and obesity on fetal development, physicians can now aid their patients in implementing lifestyle changes in order to prevent these effects. 


\section{Future Study Recommendations}

The specific aims of this study were accomplished but also led to many unanswered questions. First, it is evident that maternal HFD or and/or obesity lead to a decrease in $\alpha$ cell mass in the fetal animal. However, it is unclear what the maternal trigger is that produces this: is it hyperinsulinemia, elevated TGs or increased glucose exposure to the fetus? The cellular mechanisms that lead to the decreased mass of these cells also need to be uncovered. Likewise, the timing of $\alpha$ cell loss or decreased neogenesis or replication is not clear: what time point in pregnancy is critical for islet development? First, in order to determine the mechanisms that lead to the adaptations in fetal $\alpha$ cells, it will be necessary to establish the standard rate of $\alpha$ cell replication, neogenesis and apoptosis in CTR fetal animals. By comparing these measures to the HFD offspring, we can measure how cell cyclicity may be altered. One pilot experiment, in CTR and HFD animals, performed in the spring of 2011, was intended to measure cell replication in the fetuses. I gave CTR and HFD dams two thymidine analogs during their pregnancy. One, 5-iododeoxyuridine (Idu) was administered at the end of the first trimester, and the other, 5-chlorodeoxyuridine (Cldu), was administered at the end of the second trimester in each of these animals. The fetal animal was then collected at G130. These analogs are incorporated into a cell's DNA when the cell is dividing (during the Sphase of the cell cycle) (Teta, Rankin, Long, Stein, \& Kushner, 2007). They can then be detected by double or triple label immunohistochemistry to determine which cells underwent replication. While this experiment is still in process, we can use the pancreata from these animals to determine the number of $\alpha$ or $\beta$ cells that replicated during these time periods.

Comstock, 125 
In addition, while I have demonstrated a decrease in the $\alpha$ cell mass in the fetal offspring, the biochemical changes that occur due to a decrease in $\alpha$ cell mass in the fetal animal should also be measured. By isolating islets we can determine how the relative $\beta$ cell to $\alpha$ cell ratio affects islet function. First, I propose that whole islets be isolated from fetal animals and the basal activity of these islets should be assessed by measuring insulin and glucagon secretion in basal glucose islet media. This should be followed by stimulation of islets with glucose, insulin, glucagon and FFAs to determine their relative reactivity. I would also suggest disassociating islet cells, as previously described (Halban et al., 1982), and performing similar experiments with varying ratios of $\beta$ cells and $\alpha$ cell to determine the effect the ratio of these cells has on secretory ability.

Also, the expression of insulin mRNA was decreased in fetal HFD offspring which lead to a decrease in $\beta$ cell insulin secretion, as measured by c-peptide content. However, the changes demonstrated in other key genes do not always translate into protein changes. I recommend following these studies with both western blotting and immunohistochemistry to determine the relative levels of protein. PDXI mRNA expression was decreased in the fetal offspring; however, immunohistochemical anaylsis did not reveal any differences in protein. This indicates that there may be key protein signaling aspects that are impacted.

The second aim, to characterize the metabolic profile of the infant offspring of HFD mothers, was accomplished. However, the signaling mechanism that results in insulin resistance followed by hyperinsulinemia should be elucidated. The changes in physiological adaptation to the HFD in the post-natal animal could be clarified by 
performing clamp techniques. A hyperinsulinemic-euglycemic clamp requires a steady infusion of insulin that retains glucose levels at normal fasting concentrations. This clamp is intended to suppress hepatic glucose production but also allows determination of insulin sensitivity of peripheral tissue. The degree of insulin resistance is inversely related to glucose uptake during this protocol (McAuley et al., 2001). A second clamp technique, the hyperinsulinemic-hyperglycemic clamp provides a better measure of $\beta$ cell function. Glucose concentration is raised above basal levels by a continuous infusion of glucose then insulin is measured at various time points. Because the plasma glucose concentration is held constant, the glucose infusion rate is an index of insulin secretion and glucose metabolism (Bolli et al., 1984). These could be performed in the infant animal as early as 90 days then followed through at various time points throughout the first year of life to obtain an accurate indication of insulin sensitivity and $\beta$ cell function.

Furthermore, based on the results of my third aim, it is clear that significant islet remodeling is occurring during the post-weaning period. However, the time period of these changes and the specific physiological triggers are still unclear. The thymidine labeling protocol would be beneficial to elucidate the time period of islet changes during postnatal development. By administering the two different thymidine analogs at different time points before and after weaning, we may be able to target key stages of islet development. $\beta$ cell replication is likely a significant contributor to the changes observed in insulin secretion in juvenile animal. These studies would allow us to determine what changes are occurring during these key developmental periods. In addition to replication, neogenesis and apoptosis are also likely contributors to the islet remodeling evident in the juvenile animals. One marker of islet neogensis, which has been associated with $\beta$ cell 
mass expansion is the islet neogenesis associated protein related protein (INGAP) (Rosenberg et al., 2004). Detection of this protein by immunohistochemistry would provide some indication of the neogenesis that is occurring during the post-natal period. Another protein, Ki67, is detectable in all cell types during active phases of the cell cycle (G1, S, G2, and mitosis), but is absent from resting cells (G0) (Scholzen \& Gerdes, 2000). Immuno-detection of this protein would indicate which cells are proliferating. Co-localization of the Ki67 antibody with an INGAP antibody and a islet cell specific antibody, would lead to clarification of which cell types are undergoing neogenesis, replication or are static. A TUNEL (Terminal deoxynucleotidyl transferase dUTP nick end labeling) stain, which detects DNA fragmentation, would establish the relative level of apoptosis during the juvenile period (Gavrieli, Sherman, \& Ben-Sasson, 1992).

Finally, since, it is evident that there are different mechanisms that lead to islet expansion in the CTR/HFD versus the HFD/HFD offspring, the means by which the differential islet effects occur should be studied in the post-weaned animal. Islet isolation studies would be beneficial in the juvenile animals to determine how the changes in islets effect function. In order to determine the mechanisms that lead to islet response in these animals, I suggest stimulating islets with glucose, insulin, glucagon and FFAs and determining the relative gene expression differences between the stimulated and basally active islets. Key genes of interest would be those that were already measured, including insulin, glucagon, GK, GLUT2, Kir6.2, SUR1, and FFAR1. GLUT5, which is the glucose transporter in $\alpha$ cells (Sato et al., 1996), would also be of critical importance. Since many of the gene expression differences in the juvenile animals were inconclusive, I recommend following up on these studies by determining Comstock, 128 
the relative level of protein expression of the key genes examined within specific islet cells. Triple label immunohistochemistry with anti-insulin, anti-glucagon and a third antibody against the protein of interest would be necessary. Then sterological analysis of islet cell specificity would be required.

Comstock, 129 
Chapter 7: Summary and Discussion

Comstock, 130 
As childhood MetS is becoming an increasing concern, it is essential to establish a model that will help us determine the mechanisms that lead to this disease. Upon review, I believe my studies help to establish the juvenile HFD/HFD animals as a model of early onset MetS. I have demonstrated that maternal HFD decreases fetal $\alpha$ cell mass, which results in an increase in the $\beta$ cell to $\alpha$ cell ratio in the macaque that remains increased with continued HFD exposure (Fig. 7.1). This may be a fetal adaptation in favor of decreasing glucose production, reducing lipolysis and maintaining insulin in response to increased nutrient supply. However, by the early postnatal period, the HFD offspring become insulin resistant, which is followed by increased insulin secretion and a corresponding increase in $\beta$ cell mass. The increased $\beta$ cell to $\alpha$ cell ratio in the HFD/HFD juvenile indicates that chronic HFD exposure during the fetal and early postnatal period may lead to programmed dysfunction in the paracrinolgy of juvenile islets. By feeding these animals a HFD, they reach a pre-diabetic state before puberty that is similar to what has been demonstrated in adult insulin resistant animals (Bodkin, Metzger, \& Hansen, 1989; de Koning, et al., 1993; Hansen \& Bodkin, 1990). My results, in conjunction with the previously described prediatric NAFLD, hepatic apoptosis, and disturbances in the melanocortin system that increase HGP in these animals, indicate that these animals have an elevated risk for early-onset diabetes.

Comstock, 131 


\section{Fetal Effects of the Maternal HFD}

Epidemiological and animal studies demonstrate an association between childhood obesity and maternal obesity and/or high-fat/high calorie intake (Boney, et al., 2005; Srinivasan, et al., 2006; Whitaker, 2004). However, while the phenotype of the HFD macaque dams varies significantly, maternal sensitivity to weight gain had little effect on islet morphology or glucoregulatory function in the offspring. In fact, maternal HFD consumption was demonstrated to be the principal contributing factor to islet defects in the fetal offspring, indicating that maternal diet choices may program fetal susceptibility to islet malfunction.

The decreased $\alpha$ cell mass is a novel finding in fetal offspring of HFD mothers that may be detrimental to long term islet function, because the paracrine interactions between $\alpha$ cell and $\beta$ cells are essential for glucose homeostasis. Glucagon secretion decreases when circulating glucose levels rise. However, glucose does not have a direct inhibitory effect on $\alpha$ cells, since glucose is an essential fuel source that is used in ATP production in $\alpha$ cells. In fact, isolated $\alpha$ cells secrete glucagon in response to glucose when they are disassociated from $\beta$ cells and lack the paracrine interaction of these cells (Franklin, et al., 2005). However, it has been demonstrated the $\alpha$ cell contains an abundance of insulin receptors and recent findings have shown that insulin inhibits glucagon release from these cells. While $\alpha$ cell stimulatory pathways are still largely uncharacterized, it is evident that insulin and other $\beta$ cell secretory products lead to increased sensitivity of KATP channels, which results in $\alpha$ cell hyperpolarization and an inhibition of glucagon secretion (Leung, et al., 2006). These studies indicate that the 
paracrine interaction between $\beta$ cells and $\alpha$ cells is essential to lead to glucose inhibition of glucagon secretion (Fig. 7.2).

In contrast, glucagon has been demonstrated to have an insulin stimulatory effect (Kieffer, Heller, Unson, Weir, \& Habener, 1996). Other factors released by $\alpha$ cells, such as glucagon-like peptide-1 (GLP1) and gastric inhibitory polypeptide (GIP), have also been shown to stimulate insulin release. However, the majority of GLP1 and GIP are secreted from the intestine (Mojsov, Weir, \& Habener, 1987). When disassociated from $\alpha$ cells, $\beta$ cells have a poor insulin secretory response, despite normal glucose metabolism (Holz, et al., 1993; Pipeleers, Schuit, Van Schravendijk, \& Van de Winkel, 1985). This indicates that there is a basal level of glucagon or other $\alpha$ cell secretagogues that are required for efficient insulin release by $\beta$ cells. Also, the glucagon receptor is present on both $\beta$ cells and $10 \%$ of $\alpha$ cells (Kieffer, et al., 1996). The insulinotropic action of glucagon may be essential for maintaining basal insulin release because low levels of the peptide cause a modest, but significant, release of insulin. These results also demonstrate a clear role for paracrine regulation of $\beta$ cells insulin secretion by $\alpha$ cells (Figure 7.3).

The loss of $\alpha$ cells in the HFD fetal animal results in an elevation in the $\beta / \alpha$-ratio that perpetuates into the juvenile period. While initially this may be an adaptive, beneficial response, long-term elevation in this ratio could lead to disturbances in paracrine regulation of islets. While no studies have yet shown what the $\beta / \alpha$ cell ratio has on islet function, it is clear from the above mentioned islet paracrine interactions that a disturbance in this ratio may lead to detrimental effects in the HFD fed offspring. It is foreseeable that the disproportionate elevation in $\beta$ cells might lead to decreased glucagon 
potentiation of insulin release and inefficient insulin secretion, which is often develops in T2DM. 


\section{Post-natal Effects of the HFD}

The post-natal HFD further exacerbates the effects I demonstrated in the HFD fetus. However, in order to determine the severity of islet defects in these animals, it is necessary to compare these effects to typical T2DM progression. The progression of this disease occurs due to interplay between insulin resistance and defects in insulin secretion that ultimately leads to impaired glucose tolerance (Fig. 7.4). However, during the initial stages of the disease process, fasting insulin levels and glucose-stimulated insulin secretion are sufficient to maintain normoglycemia. As the disease progresses, insulin resistance occurs, due to contributing environmental factors, such as obesity, HFD consumption, genetic susceptibility or early-life programming effects (Erlich et al., 1993; Hales \& Barker, 1992; Hancock et al., 2008; B. B. Kahn \& Flier, 2000; Srinivasan et al., 2005). Since MetS is associated with insulin resistance and obesity, MetS often develops in subjects as a precursor to T2DM. At this stage, $\beta$ cells compensate by secreting more insulin and $\beta$ cell mass usually expands in response to the increased need for insulin production and thus normoglycemia is maintained. However, $\beta$ cell compensation eventually becomes insufficient to match insulin resistance and subjects develop impaired glucose tolerance that ultimately results in persistent hyperglycemia (Augstein \& Salzsieder, 2009; Paulsen et al., 2010). This hyperglycemia has also been demonstrated to correspond to excessive glucagon stimulation of hepatic gluconeogenesis. Ultimately, in some subjects, insulin compensation declines as a result of either defective $\beta$ cell function or a loss of $\beta$ cells (Jurgens et al., 2011; Maedler \& Donath, 2004).

In comparison, both the CTR/HFD and HFD/HFD have increased $\beta$ cell mass and elevated insulin secretion that are characteristic of the initial stages of disease 
progression, indicating that early post-natal HFD diet consumption significantly increases MetS risk. In fact, in other NHP models, insulin resistance and other MetS-like effects do not occur until at least three years of age (Choi, et al., 2011; Guardado-Mendoza et al., 2009; Kaufman, et al., 2005), but the typical onset of this disease is during middle age (15 to 20 years of age) (de Koning, et al., 1993). This suggests that HFD consumption accelerates the development of this disease in juvenile macaques. However, while both the CTR/HFD and HFD/HFD juveniles have increased $\beta$ cell mass and elevated insulin levels, maternal HFD programs the juveniles for excessive $\beta$ cell hypersecretion. This is evidenced by the fact that both the HFD/CTR and the HFD/HFD offspring have elevated insulin gene transcription. In addition, the $\beta$ cell hyper-responsiveness that is clearly evident in only the HFD/HFD offspring indicates that maternal HFD accelerates disease progression in these animals. The effect may be due to length of exposure to the HFD since the CTR/HFD offspring have only consumed the diet for 5 months. However, the maintenance of the elevated $\beta$ cell to $\alpha$ cell ratio only in the HFD/HFD juvenile indicates that maternal programming and post-weaning consumption produce a synergistic effect.

It should be noted that juvenile offspring are still immature and many effects of the post-weaning diet may also program these animals for metabolic dysfunction later in life. It will be necessary to follow these animals to adulthood to determine if maternal HFD consumption increases the incidence of obesity and T2DM in these offspring. Likewise, by placing both the CTR/CTR and the HFD/CTR animals onto a HFD as adults, we will be able to measure the relative impact of the maternal programming effect on the adult obesity onset which is so common in the world today.

Comstock, 136 
In conclusion, my studies, in concert with the in-depth characterization of these animals by other researchers my lab, have demonstrated an increased risk for MetS and T2DM in the HFD/HFD offspring. The findings are most representative of the human population today because mothers who consume a high-fat or high-calorie diet will continue to feed their children a similar diet (Clark, Goyder, Bissell, Blank, \& Peters, 2007; Johannsen, Johannsen, \& Specker, 2006; Spruijt-Metz, Lindquist, Birch, Fisher, \& Goran, 2002). The evidence that maternal HFD consumption results in islet abnormalities that are intensified by post-natal HFD consumption indicates that the early nutritional environment is a significant contributor to increase in MetS. These studies provide unique insight into the pathways that may be leading to the increase in pediatric MetS today. 
Chapter 7 Figures

Comstock, 138 
Figure 7.1: Islet Development in the CTR versus HFD Offspring

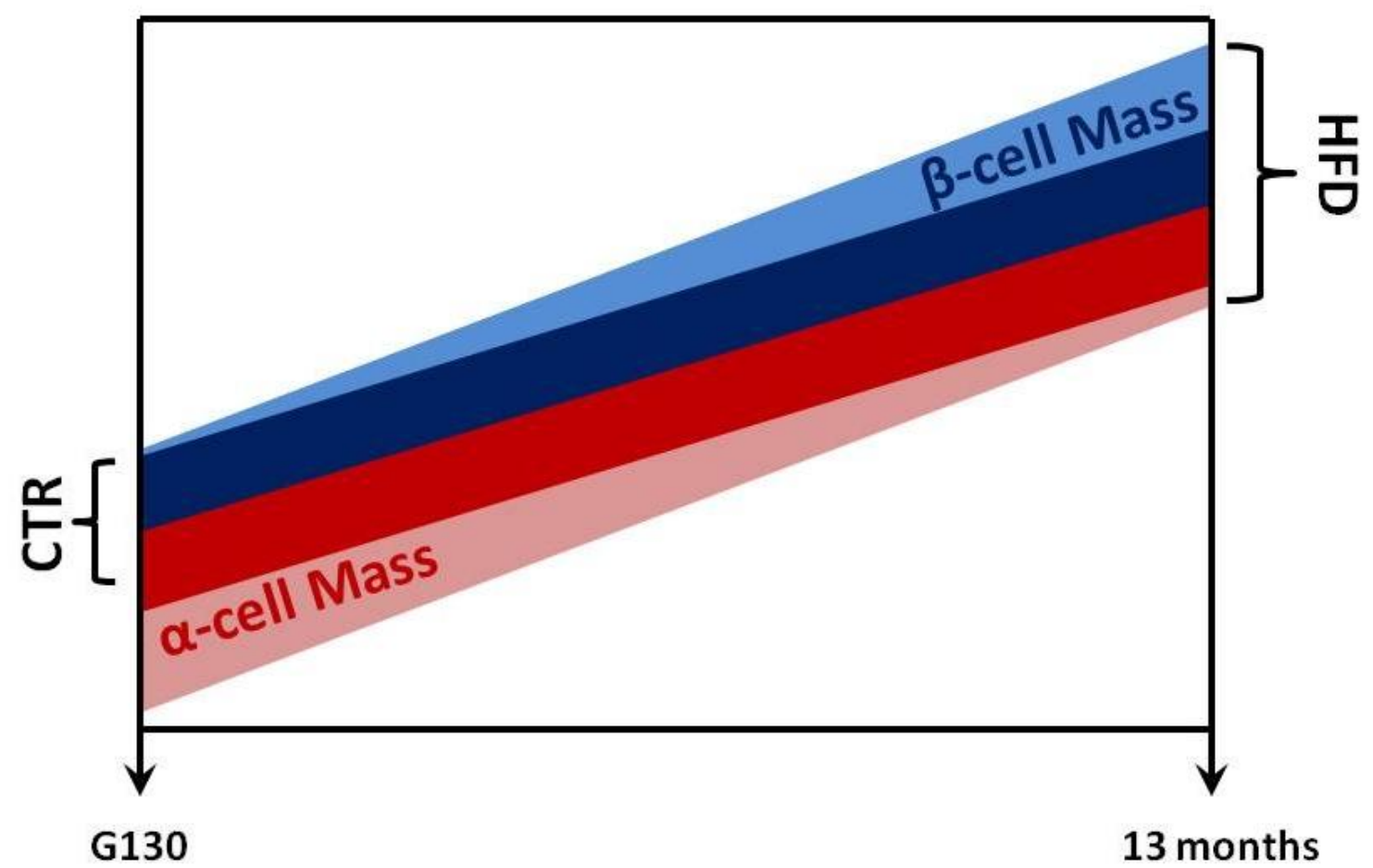

At G130, HFD offspring have an increased $\beta$ cell to $\alpha$ cell ratio, which is due to decreased $\alpha$ cell mass. As the animals age, $\alpha$ cell mass is normalized to near CTR levels but $\beta$ cell mass increases, thus maintaining the elevated $\beta$ cell to $\alpha$ cell ratio. 
Figure 7.2: Islet Paracrine Function - Glucagon Regulation of Insulin Secretion

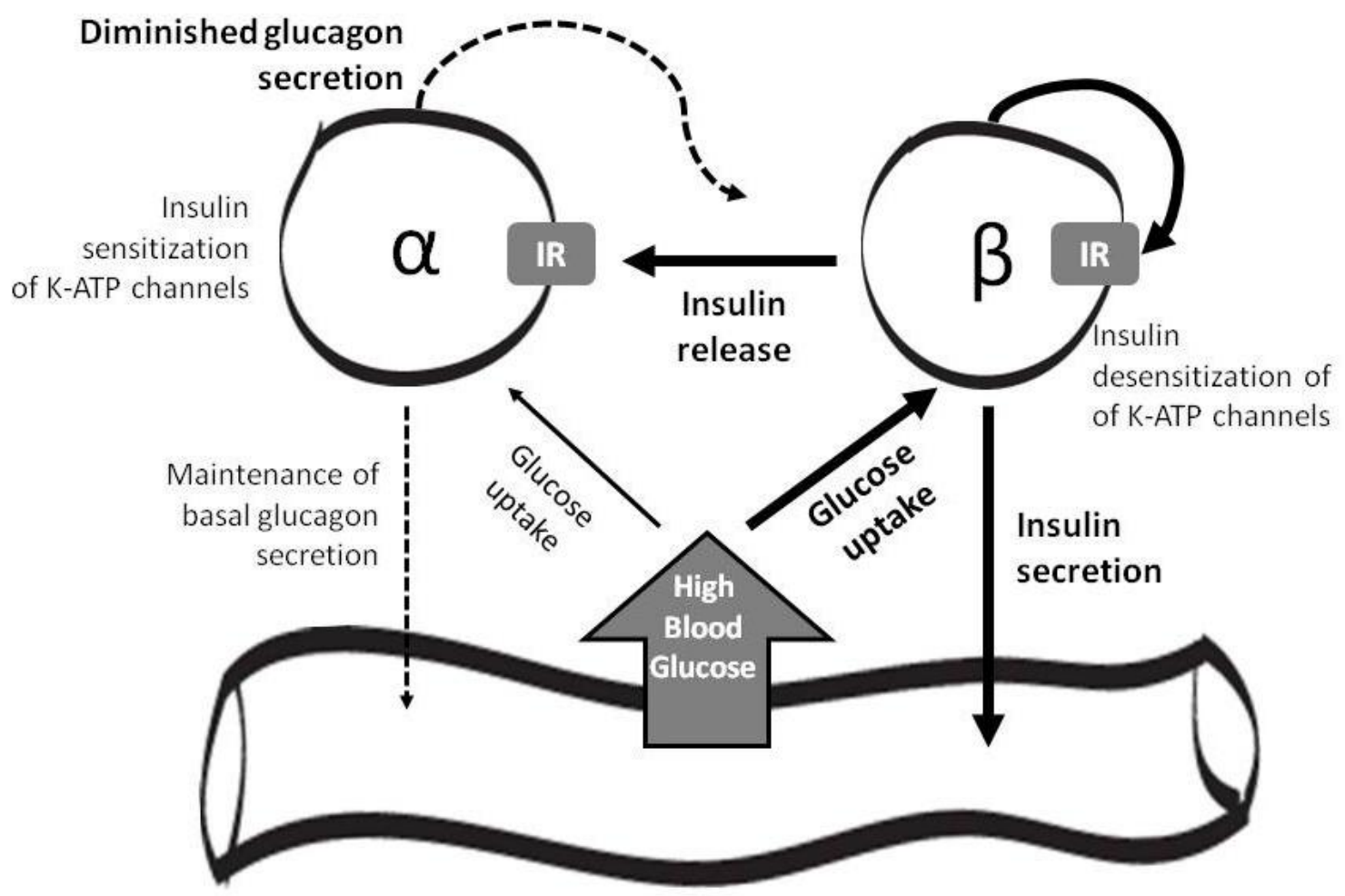

Glucose is transported into the NHP $\beta$ cell by GLUT2 and into $\alpha$ cells by GLUT5. GLUT5 has a much lower binding affinity than GLUT2 for glucose, therefore more glucose is taken up by the $\beta$ cell. Insulin is released from the $\beta$ cell in response to high glucose. Insulin signaling via the insulin receptor leads to a sensitization of -ATP channels in $\alpha$ cells. This leads to reduced glucagon secretion, because much more glucose is required to produce a sufficiently elevated ATP/ADP ratio that will depolarize the $\alpha$ cell membrane and lead to glucagon exocytosis. However, a basal level of glucagon is maintained within the intra-islet space as well as within circulation. Insulin also desensitizes KATP channels on $\beta$ cells which augments insulin secretion. (IR $=$ insulin receptor). 


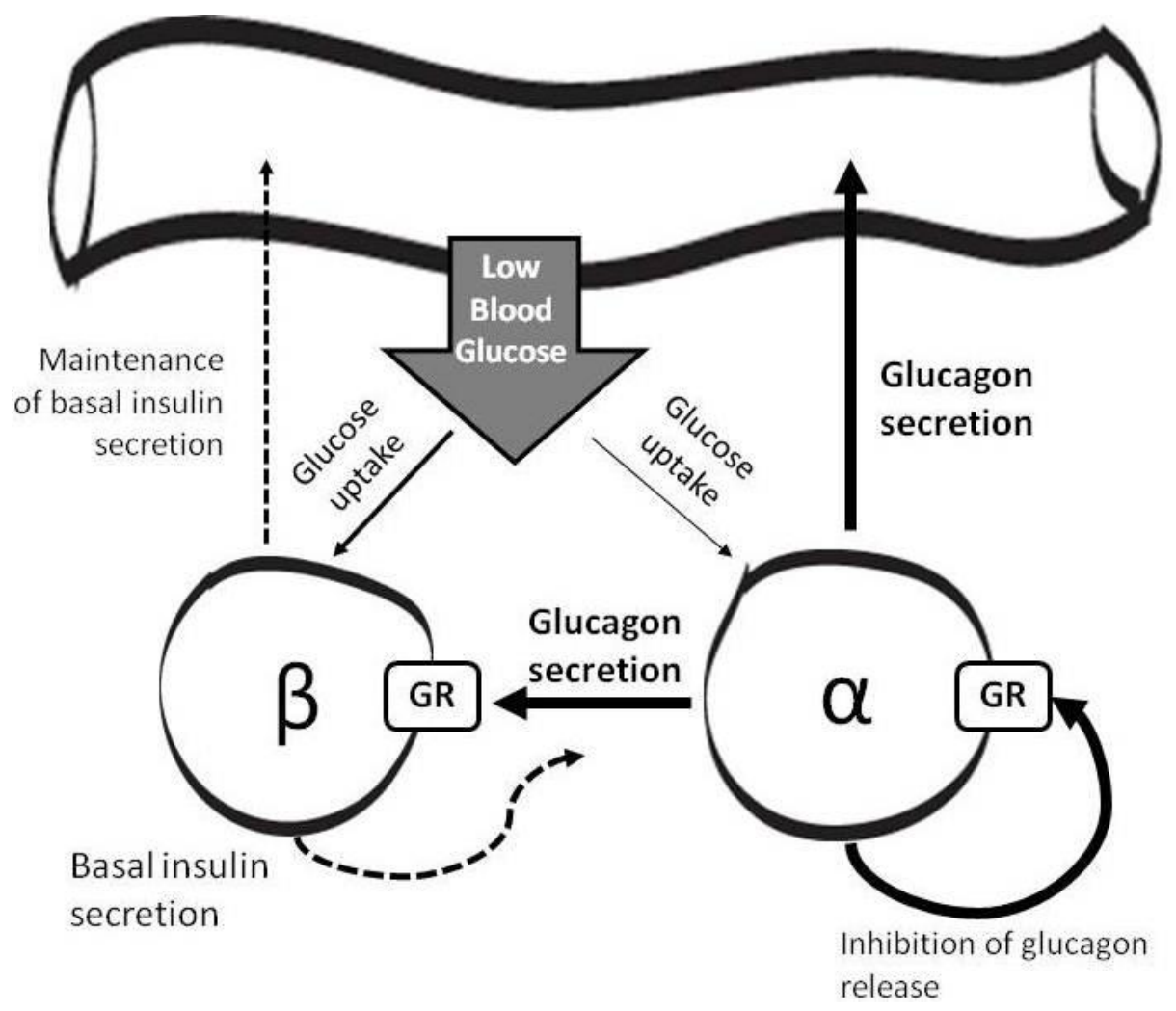

Low glucose levels lead to glucagon secretion, because the inhibitory effects from $\beta$ cells are diminished. Glucose at low levels is taken up by both $\alpha$ cell and $\beta$ cells. $\beta$ cells continue to secrete basal levels of insulin. However, without desensitization by insulin, the $\alpha$ cell K-ATP channels sense the intracellular rise in ATP caused by metabolism of glycogen stores. This causes depolarization of the cell membrane and glucagon secretion. However, high levels of $\alpha$ cell secretory products been demonstrated to have an autocrine function leading to a decline in glucagon release (Huypens, et al., 2000; Uehara et al., 2004). $\beta$ cells require signaling from $\alpha$ cell secratory products, possibly glucagon via its receptor, to efficiently release insulin. (GR = glucagon receptor). 
Figure 7.4: Pathological Progression to T2DM

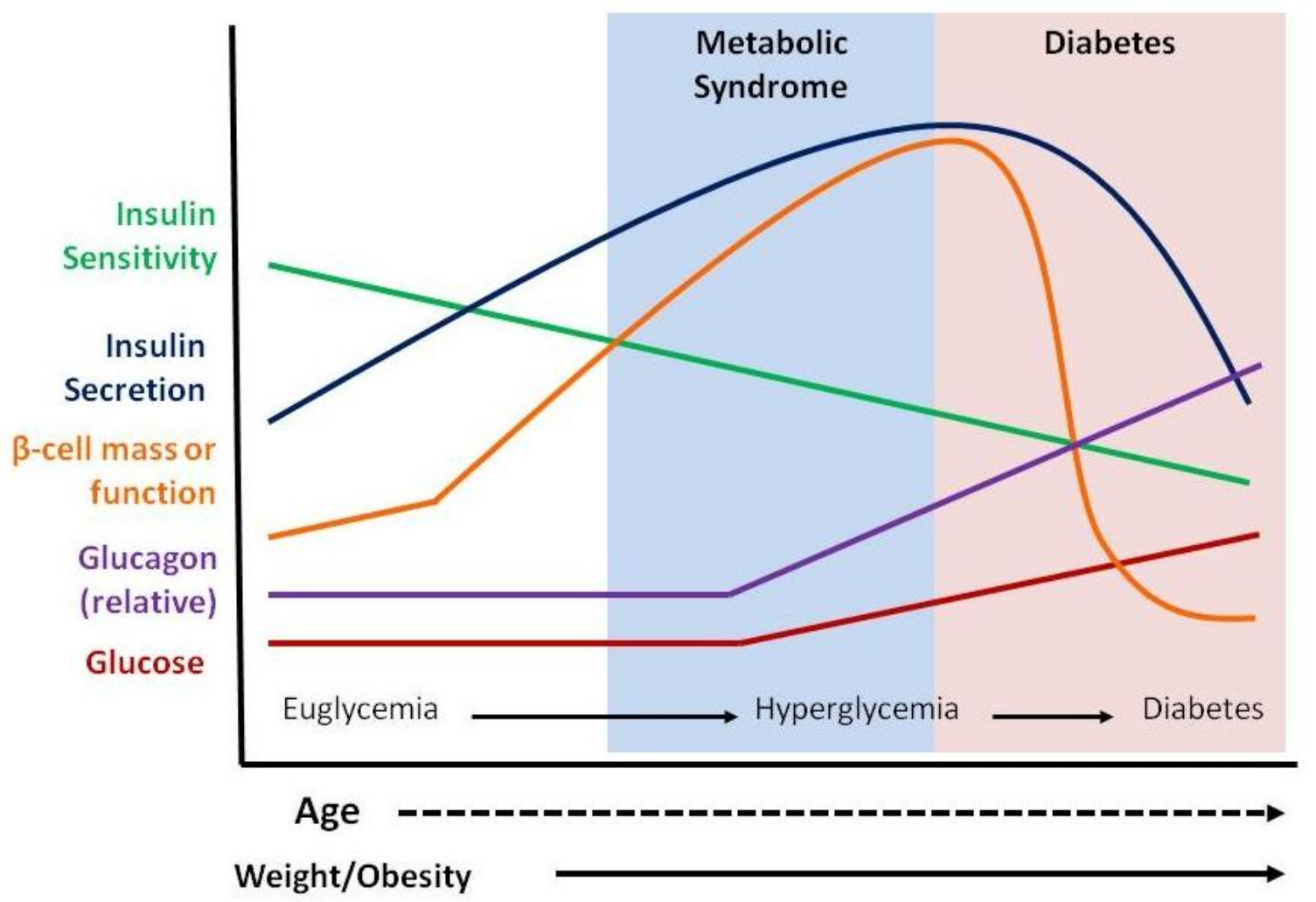

This graph represents the disease progression of MetS and T2DM. As subjects age and gain weight insulin secretion rises to match decreasing insulin sensitivity. $\beta$ cell mass increases to meet insulin secretory demand, while euglycemia is maintained. Metabolic syndrome is characterized by decreased insulin sensitivity, hyperinsulinemia, elevated $\beta$ cell mass and ultimately rising glucose and glucagon levels. Overt diabetes occurs when $\beta$ cell function declines and insulin secretion drops and hyperglycemia is uncontrolled. In some diabetic subjects, the loss of $\beta$ cell secretory capacity is associated with a loss of $\beta$ cell mass. Glucagon secretion is elevated in these subjects when compared to insulin secretion. 
Works Cited

Comstock, 143 
Aguayo-Mazzucato, C., Sanchez-Soto, C., Godinez-Puig, V., Gutierrez-Ospina, G., \& Hiriart, M. (2006). Restructuring of pancreatic islets and insulin secretion in a postnatal critical window. PLoS One, 1, e35. doi: 10.1371/journal.pone.0000035

Ahlgren, U., Jonsson, J., Jonsson, L., Simu, K., \& Edlund, H. (1998). beta-cell-specific inactivation of the mouse Ipf1/Pdx 1 gene results in loss of the beta-cell phenotype and maturity onset diabetes. Genes Dev, 12(12), 1763-1768.

Alberti, K. G., Eckel, R. H., Grundy, S. M., Zimmet, P. Z., Cleeman, J. I., Donato, K. A., et al. (2009). Harmonizing the metabolic syndrome: a joint interim statement of the International Diabetes Federation Task Force on Epidemiology and Prevention; National Heart, Lung, and Blood Institute; American Heart Association; World Heart Federation; International Atherosclerosis Society; and International Association for the Study of Obesity. Circulation, 120(16), 16401645. doi: CIRCULATIONAHA.109.192644 [pii]

10.1161/CIRCULATIONAHA.109.192644

Augstein, P., \& Salzsieder, E. (2009). Morphology of pancreatic islets: a time course of pre-diabetes in Zucker fatty rats. Methods Mol Biol, 560, 159-189. doi: 10.1007/978-1-59745-448-3_12

Barker, D. J. (1995). The fetal and infant origins of disease. Eur J Clin Invest, 25(7), 457463.

Barker, D. J., Gluckman, P. D., Godfrey, K. M., Harding, J. E., Owens, J. A., \& Robinson, J. S. (1993). Fetal nutrition and cardiovascular disease in adult life. Lancet, 341(8850), 938-941. doi: 0140-6736(93)91224-A [pii]

Barker, D. J., Winter, P. D., Osmond, C., Margetts, B., \& Simmonds, S. J. (1989). Weight in infancy and death from ischaemic heart disease. Lancet, 2(8663), 577580. doi: S0140-6736(89)90710-1 [pii]

Bayol, S. A., Simbi, B. H., \& Stickland, N. C. (2005). A maternal cafeteria diet during gestation and lactation promotes adiposity and impairs skeletal muscle development and metabolism in rat offspring at weaning. J Physiol, 567(Pt 3), 951-961. doi: jphysiol.2005.088989 [pii]

10.1113/jphysiol.2005.088989

Benoit, S. C., Air, E. L., Coolen, L. M., Strauss, R., Jackman, A., Clegg, D. J., et al. (2002). The catabolic action of insulin in the brain is mediated by melanocortins. J Neurosci, 22(20), 9048-9052. doi: 22/20/9048 [pii]

Beringue, F., Blondeau, B., Castellotti, M. C., Breant, B., Czernichow, P., \& Polak, M. (2002). Endocrine pancreas development in growth-retarded human fetuses. Diabetes, 51(2), 385-391.

Bertin, E., Gangnerau, M. N., Bellon, G., Bailbe, D., Arbelot De Vacqueur, A., \& Portha, B. (2002). Development of beta-cell mass in fetuses of rats deprived of protein and/or energy in last trimester of pregnancy. Am J Physiol Regul Integr Comp Physiol, 283(3), R623-630. doi: 10.1152/ajpregu.00037.2002

Bodkin, N. L., Metzger, B. L., \& Hansen, B. C. (1989). Hepatic glucose production and insulin sensitivity preceding diabetes in monkeys. Am J Physiol, 256(5 Pt 1), E676-681.

Bolli, G., De Feo, P., Perriello, G., De Cosmo, S., Compagnucci, P., Santeusanio, F., et al. (1984). Mechanisms of glucagon secretion during insulin-induced 
hypoglycemia in man. Role of the beta cell and arterial hyperinsulinemia. J Clin Invest, 73(4), 917-922. doi: 10.1172/JCI111315

Boney, C. M., Verma, A., Tucker, R., \& Vohr, B. R. (2005). Metabolic syndrome in childhood: association with birth weight, maternal obesity, and gestational diabetes mellitus. Pediatrics, 115(3), e290-296. doi: 115/3/e290 [pii]

10.1542/peds.2004-1808

Bonner-Weir, S. (2001). beta-cell turnover: its assessment and implications. Diabetes, 50 Suppl 1, S20-24.

Bonora, E., Targher, G., Alberiche, M., Bonadonna, R. C., Saggiani, F., Zenere, M. B., et al. (2000). Homeostasis model assessment closely mirrors the glucose clamp technique in the assessment of insulin sensitivity: studies in subjects with various degrees of glucose tolerance and insulin sensitivity. Diabetes Care, 23(1), 57-63.

Briscoe, C. P., Tadayyon, M., Andrews, J. L., Benson, W. G., Chambers, J. K., Eilert, M. M., et al. (2003). The orphan G protein-coupled receptor GPR40 is activated by medium and long chain fatty acids. J Biol Chem, 278(13), 11303-11311. doi: 10.1074/jbc.M211495200

M211495200 [pii]

Brissova, M., Fowler, M. J., Nicholson, W. E., Chu, A., Hirshberg, B., Harlan, D. M., et al. (2005). Assessment of human pancreatic islet architecture and composition by laser scanning confocal microscopy. J Histochem Cytochem, 53(9), 1087-1097. doi: jhc.5C6684.2005 [pii]

10.1369/jhc.5C6684.2005

Brissova, M., Shiota, M., Nicholson, W. E., Gannon, M., Knobel, S. M., Piston, D. W., et al. (2002). Reduction in pancreatic transcription factor PDX-1 impairs glucosestimulated insulin secretion. J Biol Chem, 277(13), 11225-11232. doi: 10.1074/jbc.M111272200

M111272200 [pii]

Buckley, A. J., Keseru, B., Briody, J., Thompson, M., Ozanne, S. E., \& Thompson, C. H. (2005). Altered body composition and metabolism in the male offspring of high fat-fed rats. Metabolism, 54(4), 500-507. doi: S0026049504004275 [pii]

10.1016/j.metabol.2004.11.003

Burton, B. T., Foster, W. R., Hirsch, J., \& Van Itallie, T. B. (1985). Health implications of obesity: an NIH Consensus Development Conference. Int J Obes, 9(3), 155170.

Buteau, J., Roduit, R., Susini, S., \& Prentki, M. (1999). Glucagon-like peptide-1 promotes DNA synthesis, activates phosphatidylinositol 3-kinase and increases transcription factor pancreatic and duodenal homeobox gene 1 (PDX-1) DNA binding activity in beta (INS-1)-cells. Diabetologia, 42(7), 856-864. doi: $10.1007 / \mathrm{s} 001250051238$

Butler, A. E., Cao-Minh, L., Galasso, R., Rizza, R. A., Corradin, A., Cobelli, C., et al. (2010). Adaptive changes in pancreatic beta cell fractional area and beta cell turnover in human pregnancy. Diabetologia, 53(10), 2167-2176. doi: $10.1007 / \mathrm{s} 00125-010-1809-6$ 
Butler, A. E., Janson, J., Bonner-Weir, S., Ritzel, R., Rizza, R. A., \& Butler, P. C. (2003). Beta-cell deficit and increased beta-cell apoptosis in humans with type 2 diabetes. Diabetes, 52(1), 102-110.

Cabrera, O., Berman, D. M., Kenyon, N. S., Ricordi, C., Berggren, P. O., \& Caicedo, A. (2006). The unique cytoarchitecture of human pancreatic islets has implications for islet cell function. Proc Natl Acad Sci U S A, 103(7), 2334-2339. doi: 0510790103 [pii]

10.1073/pnas.0510790103

Carriere, F., Barrowman, J. A., Verger, R., \& Laugier, R. (1993). Secretion and contribution to lipolysis of gastric and pancreatic lipases during a test meal in humans. Gastroenterology, 105(3), 876-888. doi: S0016508593002999 [pii]

Cattaneo, A., Monasta, L., Stamatakis, E., Lioret, S., Castetbon, K., Frenken, F., et al. (2010). Overweight and obesity in infants and pre-school children in the European Union: a review of existing data. Obes Rev, 11(5), 389-398. doi: OBR639 [pii]

10.1111/j.1467-789X.2009.00639.x

Celermajer, D. S., Sorensen, K. E., Bull, C., Robinson, J., \& Deanfield, J. E. (1994). Endothelium-dependent dilation in the systemic arteries of asymptomatic subjects relates to coronary risk factors and their interaction. J Am Coll Cardiol, 24(6), 1468-1474. doi: 0735-1097(94)90141-4 [pii]

Cerf, M. E., Muller, C. J., Du Toit, D. F., Louw, J., \& Wolfe-Coote, S. A. (2006). Hyperglycaemia and reduced glucokinase expression in weanling offspring from dams maintained on a high-fat diet. Br J Nutr, 95(2), 391-396. doi: S0007114506000523 [pii]

Cerf, M. E., Williams, K., Nkomo, X. I., Muller, C. J., Du Toit, D. F., Louw, J., et al. (2005). Islet cell response in the neonatal rat after exposure to a high-fat diet during pregnancy. Am J Physiol Regul Integr Comp Physiol, 288(5), R1122-1128. doi: 00335.2004 [pii]

10.1152/ajpregu.00335.2004

Chakravarty, K., Cassuto, H., Reshef, L., \& Hanson, R. W. (2005). Factors that control the tissue-specific transcription of the gene for phosphoenolpyruvate carboxykinase-C. Crit Rev Biochem Mol Biol, 40(3), 129-154. doi: 40/3/129 [pii] 10.1080/10409230590935479

Chen, H., Simar, D., Lambert, K., Mercier, J., \& Morris, M. J. (2008). Maternal and postnatal overnutrition differentially impact appetite regulators and fuel metabolism. Endocrinology, 149(11), 5348-5356. doi: en.2008-0582 [pii]

10.1210/en.2008-0582

Chen, Y. D., Golay, A., Swislocki, A. L., \& Reaven, G. M. (1987). Resistance to insulin suppression of plasma free fatty acid concentrations and insulin stimulation of glucose uptake in noninsulin-dependent diabetes mellitus. J Clin Endocrinol Metab, 64(1), 17-21.

Choi, J., Li, C., McDonald, T. J., Comuzzie, A., Mattern, V., \& Nathanielsz, P. W. (2011). Emergence of insulin resistance in juvenile baboon offspring of mothers exposed to moderate maternal nutrient reduction. Am J Physiol Regul Integr Comp Physiol, 301(3), R757-762. doi: ajpregu.00051.2011 [pii]

10.1152/ajpregu.00051.2011

Comstock, 146 
Clark, H. R., Goyder, E., Bissell, P., Blank, L., \& Peters, J. (2007). How do parents' child-feeding behaviours influence child weight? Implications for childhood obesity policy. J Public Health (Oxf), 29(2), 132-141. doi: fdm012 [pii]

10.1093/pubmed/fdm012

Comstock, S. M. (2012). High fat diet during perinatal development reduces $\alpha$-cell mass and leads to early insulin resistance in the infantile nonhuman primate.

Consoli, A., Nurjhan, N., Capani, F., \& Gerich, J. (1989). Predominant role of gluconeogenesis in increased hepatic glucose production in NIDDM. Diabetes, 38(5), 550-557.

Cook, S., Weitzman, M., Auinger, P., Nguyen, M., \& Dietz, W. H. (2003). Prevalence of a metabolic syndrome phenotype in adolescents: findings from the third National Health and Nutrition Examination Survey, 1988-1994. Arch Pediatr Adolesc Med, 157(8), 821-827. doi: 10.1001/archpedi.157.8.821

$157 / 8 / 821$ [pii]

Cushman, S. W., \& Wardzala, L. J. (1980). Potential mechanism of insulin action on glucose transport in the isolated rat adipose cell. Apparent translocation of intracellular transport systems to the plasma membrane. J Biol Chem, 255(10), 4758-4762.

Cusi, K., Kashyap, S., Gastaldelli, A., Bajaj, M., \& Cersosimo, E. (2007). Effects on insulin secretion and insulin action of a 48-h reduction of plasma free fatty acids with acipimox in nondiabetic subjects genetically predisposed to type 2 diabetes. Am J Physiol Endocrinol Metab, 292(6), E1775-1781. doi: 00624.2006 [pii]

10.1152/ajpendo.00624.2006

Dahri, S., Snoeck, A., Reusens-Billen, B., Remacle, C., \& Hoet, J. J. (1991). Islet function in offspring of mothers on low-protein diet during gestation. Diabetes, 40 Suppl 2, 115-120.

Darp, R. A., de Boo, H. A., Phua, H. H., Oliver, M. H., Derraik, J. G., Harding, J. E., et al. (2010). Differential regulation of igf1 and igf1r mRNA levels in the two hepatic lobes following intrauterine growth restriction and its treatment with intraamniotic insulin-like growth factor-1 in ovine fetuses. Reprod Fertil Dev, 22(8), 1188-1197. doi: RD09292 [pii]

10.1071/RD09292

de Koning, E. J., Bodkin, N. L., Hansen, B. C., \& Clark, A. (1993). Diabetes mellitus in Macaca mulatta monkeys is characterised by islet amyloidosis and reduction in beta-cell population. Diabetologia, 36(5), 378-384.

De Vos, A., Heimberg, H., Quartier, E., Huypens, P., Bouwens, L., Pipeleers, D., et al. (1995). Human and rat beta cells differ in glucose transporter but not in glucokinase gene expression. J Clin Invest, 96(5), 2489-2495.

Del Guerra, S., Bugliani, M., D'Aleo, V., Del Prato, S., Boggi, U., Mosca, F., et al. (2010). G-protein-coupled receptor 40 (GPR40) expression and its regulation in human pancreatic islets: the role of type 2 diabetes and fatty acids. Nutr Metab Cardiovasc Dis, 20(1), 22-25. doi: S0939-4753(09)00039-8 [pii]

10.1016/j.numecd.2009.02.008

Diagnosis and classification of diabetes mellitus. (2011). Diabetes Care, 34 Suppl 1, S6269. doi: 34/Supplement_1/S62 [pii] 
10.2337/dc11-S062

Drury, D. R., Wick, A. N., Bancroft, R. W., \& Mac, K. E. (1951). Glucose utilization by the extra-hepatic tissues. Am J Physiol, 164(1), 207-212.

Dumortier, O., Blondeau, B., Duvillie, B., Reusens, B., Breant, B., \& Remacle, C. (2007). Different mechanisms operating during different critical time-windows reduce rat fetal beta cell mass due to a maternal low-protein or low-energy diet. Diabetologia, 50(12), 2495-2503. doi: 10.1007/s00125-007-0811-0

Dunn, G. A., \& Bale, T. L. (2009). Maternal high-fat diet promotes body length increases and insulin insensitivity in second-generation mice. Endocrinology, 150(11), 4999-5009. doi: en.2009-0500 [pii]

10.1210/en.2009-0500

Dzeja, P. P., \& Terzic, A. (2003). Phosphotransfer networks and cellular energetics. $J$ Exp Biol, 206(Pt 12), 2039-2047.

Dzeja, P. P., Zeleznikar, R. J., \& Goldberg, N. D. (1998). Adenylate kinase: kinetic behavior in intact cells indicates it is integral to multiple cellular processes. $\mathrm{Mol}$ Cell Biochem, 184(1-2), 169-182.

Ehses, J. A., Ellingsgaard, H., Boni-Schnetzler, M., \& Donath, M. Y. (2009). Pancreatic islet inflammation in type 2 diabetes: from alpha and beta cell compensation to dysfunction. Arch Physiol Biochem, 115(4), 240-247. doi: 10.1080/13813450903025879

Elghazi, L., Cras-Meneur, C., Czernichow, P., \& Scharfmann, R. (2002). Role for FGFR2IIIb-mediated signals in controlling pancreatic endocrine progenitor cell proliferation. Proc Natl Acad Sci U S A, 99(6), 3884-3889. doi: 10.1073/pnas.062321799

062321799 [pii]

Ellingsgaard, H., Hauselmann, I., Schuler, B., Habib, A. M., Baggio, L. L., Meier, D. T., et al. (2011). Interleukin-6 enhances insulin secretion by increasing glucagon-like peptide-1 secretion from L cells and alpha cells. Nat Med, 17(11), 1481-1489. doi: $\mathrm{nm} .2513$ [pii]

$10.1038 / \mathrm{nm} .2513$

Enriori, P. J., Evans, A. E., Sinnayah, P., Jobst, E. E., Tonelli-Lemos, L., Billes, S. K., et al. (2007). Diet-induced obesity causes severe but reversible leptin resistance in arcuate melanocortin neurons. Cell Metab, 5(3), 181-194.

Erlich, H. A., Zeidler, A., Chang, J., Shaw, S., Raffel, L. J., Klitz, W., et al. (1993). HLA class II alleles and susceptibility and resistance to insulin dependent diabetes mellitus in Mexican-American families. Nat Genet, 3(4), 358-364. doi: 10.1038/ng0493-358

Estrany, M. E., Proenza, A. M., Llado, I., \& Gianotti, M. (2011). Isocaloric intake of a high-fat diet modifies adiposity and lipid handling in a sex dependent manner in rats. Lipids Health Dis, 10, 52. doi: 1476-511X-10-52 [pii]

10.1186/1476-511X-10-52

Fahy, A. (2009). Vascularization of the pancreas and islets of langerhans in intrauterine growth restricted fetuses and lambs. BS Bachelor, University of Arizona, Tucson. 
Fan, L., Lindsley, R., Comstock, S. M., Takahashi, D., Evans, A. E., Thornburg, K. L., et al. (2012). Maternal High-Fat Diet Programs Endothelial Dysfunction in the Aorta of Juvenile Nonhuman Primates. International Journal of Obesity, In press.

Farley, D., Tejero, M. E., Comuzzie, A. G., Higgins, P. B., Cox, L., Werner, S. L., et al. (2009). Feto-placental adaptations to maternal obesity in the baboon. Placenta, 30(9), 752-760. doi: S0143-4004(09)00206-9 [pii]

10.1016/j.placenta.2009.06.007

Ferezou-Viala, J., Roy, A. F., Serougne, C., Gripois, D., Parquet, M., Bailleux, V., et al. (2007). Long-term consequences of maternal high-fat feeding on hypothalamic leptin sensitivity and diet-induced obesity in the offspring. Am J Physiol Regul Integr Comp Physiol, 293(3), R1056-1062. doi: 00117.2007 [pii]

10.1152/ajpregu.00117.2007

Finucane, M. M., Stevens, G. A., Cowan, M. J., Danaei, G., Lin, J. K., Paciorek, C. J., et al. National, regional, and global trends in body-mass index since 1980: systematic analysis of health examination surveys and epidemiological studies with 960 country-years and 9.1 million participants. The Lancet, 377(9765), 557567. doi: 10.1016/s0140-6736(10)62037-5

Fioramonti, X., Contie, S., Song, Z., Routh, V. H., Lorsignol, A., \& Penicaud, L. (2007). Characterization of glucosensing neuron subpopulations in the arcuate nucleus: integration in neuropeptide $\mathrm{Y}$ and pro-opio melanocortin networks? Diabetes, 56(5), 1219-1227. doi: db06-0567 [pii]

$10.2337 / \mathrm{db} 06-0567$

Flodgren, E., Olde, B., Meidute-Abaraviciene, S., Winzell, M. S., Ahren, B., \& Salehi, A. (2007). GPR40 is expressed in glucagon producing cells and affects glucagon secretion. Biochem Biophys Res Commun, 354(1), 240-245. doi: S0006291X(06)02867-1 [pii]

10.1016/j.bbrc.2006.12.193

Ford, E. S., Giles, W. H., \& Dietz, W. H. (2002). Prevalence of the metabolic syndrome among US adults: findings from the third National Health and Nutrition Examination Survey. JAMA, 287(3), 356-359. doi: jbr10281 [pii]

Ford, S. P., Zhang, L., Zhu, M., Miller, M. M., Smith, D. T., Hess, B. W., et al. (2009). Maternal obesity accelerates fetal pancreatic beta-cell but not alpha-cell development in sheep: prenatal consequences. Am J Physiol Regul Integr Comp Physiol, 297(3), R835-843. doi: 00072.2009 [pii]

10.1152/ajpregu.00072.2009

Forsen, T., Eriksson, J. G., Tuomilehto, J., Teramo, K., Osmond, C., \& Barker, D. J. (1997). Mother's weight in pregnancy and coronary heart disease in a cohort of Finnish men: follow up study. Bmj, 315(7112), 837-840.

Fowden, A. L., \& Hill, D. J. (2001). Intra-uterine programming of the endocrine pancreas. Br Med Bull, 60, 123-142.

Fowden, A. L., Mijovic, J., \& Silver, M. (1993). The effects of cortisol on hepatic and renal gluconeogenic enzyme activities in the sheep fetus during late gestation. $J$ Endocrinol, 137(2), 213-222. 
Franklin, I., Gromada, J., Gjinovci, A., Theander, S., \& Wollheim, C. B. (2005). Betacell secretory products activate alpha-cell ATP-dependent potassium channels to inhibit glucagon release. Diabetes, 54(6), 1808-1815. doi: 54/6/1808 [pii]

Frias, A. E., Morgan, T. K., Evans, A. E., Rasanen, J., Oh, K. Y., Thornburg, K. L., et al. Maternal high-fat diet disturbs uteroplacental hemodynamics and increases the frequency of stillbirth in a nonhuman primate model of excess nutrition. Endocrinology, 152(6), 2456-2464.

Frias, A. E., Morgan, T. K., Evans, A. E., Rasanen, J., Oh, K. Y., Thornburg, K. L., et al. (2011). Maternal high-fat diet disturbs uteroplacental hemodynamics and increases the frequency of stillbirth in a nonhuman primate model of excess nutrition. Endocrinology, 152(6), 2456-2464. doi: en.2010-1332 [pii]

10.1210/en.2010-1332

Ganong, W. F. (2007). Review of Medical Physiology (22nd ed.). Columbus, OH: McGraw Hill.

Garofano, A., Czernichow, P., \& Breant, B. (1997). In utero undernutrition impairs rat beta-cell development. Diabetologia, 40(10), 1231-1234. doi: 10.1007/s001250050812

Garofano, A., Czernichow, P., \& Breant, B. (1998). Beta-cell mass and proliferation following late fetal and early postnatal malnutrition in the rat. Diabetologia, 41(9), 1114-1120. doi: 10.1007/s001250051038

Garofano, A., Czernichow, P., \& Breant, B. (1999). Effect of ageing on beta-cell mass and function in rats malnourished during the perinatal period. Diabetologia, 42(6), 711-718. doi: 10.1007/s001250051219

Gatford, K. L., Mohammad, S. N., Harland, M. L., De Blasio, M. J., Fowden, A. L., Robinson, J. S., et al. (2008). Impaired beta-cell function and inadequate compensatory increases in beta-cell mass after intrauterine growth restriction in sheep. Endocrinology, 149(10), 5118-5127. doi: en.2008-0233 [pii]

10.1210/en.2008-0233

Gavrieli, Y., Sherman, Y., \& Ben-Sasson, S. A. (1992). Identification of programmed cell death in situ via specific labeling of nuclear DNA fragmentation. J Cell Biol, 119(3), 493-501.

George, L. A., Uthlaut, A. B., Long, N. M., Zhang, L., Ma, Y., Smith, D. T., et al. (2010). Different levels of overnutrition and weight gain during pregnancy have differential effects on fetal growth and organ development. Reprod Biol Endocrinol, 8, 75. doi: 1477-7827-8-75 [pii]

10.1186/1477-7827-8-75

Georgia, S., \& Bhushan, A. (2004). Beta cell replication is the primary mechanism for maintaining postnatal beta cell mass. J Clin Invest, 114(7), 963-968. doi: 10.1172/JCI22098

Gerich, J. E. (1976). Alpha cell function in diabetes mellitus. Metabolism, 25(11 Suppl 1), 1513-1516.

Glavas, M. M., Kirigiti, M. A., Xiao, X. Q., Enriori, P. J., Fisher, S. K., Evans, A. E., et al. (2010). Early overnutrition results in early-onset arcuate leptin resistance and increased sensitivity to high-fat diet. Endocrinology, 151(4), 1598-1610. doi: en.2009-1295 [pii] 
10.1210/en.2009-1295

Gomez-Perez, Y., Gianotti, M., Llado, I., \& Proenza, A. M. (2011). Sex-dependent effects of high-fat-diet feeding on rat pancreas oxidative stress. Pancreas, 40(5), 682-688. doi: 10.1097/MPA.0b013e31821f2645

Grant, W. F., Gillingham, M. B., Batra, A. K., Fewkes, N. M., Comstock, S. M., Takahashi, D., et al. Maternal high fat diet is associated with decreased plasma n3 fatty acids and fetal hepatic apoptosis in nonhuman primates. PLoS One, 6(2), e17261.

Grant, W. F., Gillingham, M. B., Batra, A. K., Fewkes, N. M., Comstock, S. M., Takahashi, D., et al. (2011). Maternal high fat diet is associated with decreased plasma n-3 fatty acids and fetal hepatic apoptosis in nonhuman primates. PLoS One, 6(2), e17261. doi: 10.1371/journal.pone.0017261

Grayson, B. E., Levasseur, P. R., Williams, S. M., Smith, M. S., Marks, D. L., \& Grove, K. L. Changes in melanocortin expression and inflammatory pathways in fetal offspring of nonhuman primates fed a high-fat diet. Endocrinology, 151(4), 16221632.

Grayson, B. E., Levasseur, P. R., Williams, S. M., Smith, M. S., Marks, D. L., \& Grove, K. L. (2010). Changes in melanocortin expression and inflammatory pathways in fetal offspring of nonhuman primates fed a high-fat diet. Endocrinology, 151(4), 1622-1632. doi: en.2009-1019 [pii]

10.1210/en.2009-1019

Groop, L. C., Bonadonna, R. C., Simonson, D. C., Petrides, A. S., Shank, M., \& DeFronzo, R. A. (1992). Effect of insulin on oxidative and nonoxidative pathways of free fatty acid metabolism in human obesity. Am J Physiol, 263(1 Pt 1), E7984.

Grulet, H., Durlach, V., Hecart, A. C., Gross, A., \& Leutenegger, M. (1993). Study of the rate of early glucose disappearance following insulin injection: insulin sensitivity index. Diabetes Res Clin Pract, 20(3), 201-207. doi: 0168-8227(93)90079-K [pii]

Grundy, S. M. (2006). Does a diagnosis of metabolic syndrome have value in clinical practice? Am J Clin Nutr, 83(6), 1248-1251. doi: 83/6/1248 [pii]

Grundy, S. M., Cleeman, J. I., Daniels, S. R., Donato, K. A., Eckel, R. H., Franklin, B. A., et al. (2005). Diagnosis and management of the metabolic syndrome: an American Heart Association/National Heart, Lung, and Blood Institute Scientific Statement. Circulation, 112(17), 2735-2752. doi:

CIRCULATIONAHA.105.169404 [pii]

10.1161/CIRCULATIONAHA.105.169404

Guardado-Mendoza, R., Davalli, A. M., Chavez, A. O., Hubbard, G. B., Dick, E. J., Majluf-Cruz, A., et al. (2009). Pancreatic islet amyloidosis, beta-cell apoptosis, and alpha-cell proliferation are determinants of islet remodeling in type-2 diabetic baboons. Proc Natl Acad Sci U S A, 106(33), 13992-13997. doi: 0906471106 [pii] 10.1073/pnas.0906471106

Guiot, Y., Sempoux, C., Moulin, P., \& Rahier, J. (2001). No decrease of the beta-cell mass in type 2 diabetic patients. Diabetes, 50 Suppl 1, S188. 
Guo, S. S., Wu, W., Chumlea, W. C., \& Roche, A. F. (2002). Predicting overweight and obesity in adulthood from body mass index values in childhood and adolescence. Am J Clin Nutr, 76(3), 653-658.

Gupta, R. K., Vatamaniuk, M. Z., Lee, C. S., Flaschen, R. C., Fulmer, J. T., Matschinsky, F. M., et al. (2005). The MODY1 gene HNF-4alpha regulates selected genes involved in insulin secretion. J Clin Invest, 115(4), 1006-1015.

Haffner, S. M., Valdez, R. A., Hazuda, H. P., Mitchell, B. D., Morales, P. A., \& Stern, M. P. (1992). Prospective analysis of the insulin-resistance syndrome (syndrome X). Diabetes, 41(6), 715-722.

Halban, P. A., Wollheim, C. B., Blondel, B., Meda, P., Niesor, E. N., \& Mintz, D. H. (1982). The possible importance of contact between pancreatic islet cells for the control of insulin release. Endocrinology, 111(1), 86-94.

Hales, C. N., \& Barker, D. J. (1992). Type 2 (non-insulin-dependent) diabetes mellitus: the thrifty phenotype hypothesis. Diabetologia, 35(7), 595-601.

Hales, C. N., Barker, D. J., Clark, P. M., Cox, L. J., Fall, C., Osmond, C., et al. (1991). Fetal and infant growth and impaired glucose tolerance at age 64. BMJ, 303(6809), 1019-1022.

Hall, A. C. G. a. J. E. (2000). Textbook of Medical Physiology (10 ed.). Philadelphia, PA: WB Saunder Company.

Hamada, Y. (1999). Adolescent Growth and Development in Japanese Macaques (Macacafuscata): Punctuated Adolescent Growth Spurt by Season. Primates, 40(3), 14.

Hancock, C. R., Han, D. H., Chen, M., Terada, S., Yasuda, T., Wright, D. C., et al. (2008). High-fat diets cause insulin resistance despite an increase in muscle mitochondria. Proc Natl Acad Sci U S A, 105(22), 7815-7820. doi: 0802057105 [pii]

10.1073/pnas.0802057105

Hanley, S. C., Austin, E., Assouline-Thomas, B., Kapeluto, J., Blaichman, J., Moosavi, M., et al. (2010). \{beta\}-Cell mass dynamics and islet cell plasticity in human type 2 diabetes. Endocrinology, 151(4), 1462-1472. doi: en.2009-1277 [pii]

10.1210/en.2009-1277

Hansen, B. C., \& Bodkin, N. L. (1986). Heterogeneity of insulin responses: phases leading to type 2 (non-insulin-dependent) diabetes mellitus in the rhesus monkey. Diabetologia, 29(10), 713-719.

Hansen, B. C., \& Bodkin, N. L. (1990). Beta-cell hyperresponsiveness: earliest event in development of diabetes in monkeys. Am J Physiol, $259(3 \mathrm{Pt}$ 2), R612-617.

Hayama, S., Nakiri, S., \& Konno, F. (2011). Pregnancy rate and conception date in a wild population of Japanese monkeys. J Vet Med Sci, 73(6), 809-812. doi: JST.JSTAGE/jvms/10-0420 [pii]

Hegyi, P., Maleth, J., Venglovecz, V., \& Rakonczay, Z., Jr. (2011). Pancreatic ductal bicarbonate secretion: challenge of the acinar Acid load. Front Physiol, 2, 36. doi: 10.3389/fphys.2011.00036

Heimberg, H., De Vos, A., Moens, K., Quartier, E., Bouwens, L., Pipeleers, D., et al. (1996). The glucose sensor protein glucokinase is expressed in glucagonproducing alpha-cells. Proc Natl Acad Sci U S A, 93(14), 7036-7041.

Comstock, 152 
Hill, D. J. (2005). Development of the endocrine pancreas. Rev Endocr Metab Disord, 6(3), 229-238. doi: 10.1007/s11154-005-3054-8

Holz, G. G. t., Kuhtreiber, W. M., \& Habener, J. F. (1993). Pancreatic beta-cells are rendered glucose-competent by the insulinotropic hormone glucagon-like peptide1(7-37). Nature, 361(6410), 362-365. doi: 10.1038/361362a0

Howie, G. J., Sloboda, D. M., Kamal, T., \& Vickers, M. H. (2009). Maternal nutritional history predicts obesity in adult offspring independent of postnatal diet. J Physiol, 587(Pt 4), 905-915. doi: jphysiol.2008.163477 [pii]

10.1113/jphysiol.2008.163477

Huypens, P., Ling, Z., Pipeleers, D., \& Schuit, F. (2000). Glucagon receptors on human islet cells contribute to glucose competence of insulin release. Diabetologia, 43(8), 1012-1019. doi: 10.1007/s001250051484

Isa, T., Yamane, I., Hamai, M., \& Inagaki, H. (2009). Japanese macaques as laboratory animals. Exp Anim, 58(5), 451-457. doi: JST.JSTAGE/expanim/58.451 [pii]

Iynedjian, P. B. (1993). Mammalian glucokinase and its gene. Biochem J, 293 ( Pt 1), 113.

Johannsen, D. L., Johannsen, N. M., \& Specker, B. L. (2006). Influence of parents' eating behaviors and child feeding practices on children's weight status. Obesity (Silver Spring), 14(3), 431-439. doi: 14/3/431 [pii]

10.1038/oby.2006.57

Johansson, K. A., \& Grapin-Botton, A. (2002). Development and diseases of the pancreas. Clin Genet, 62(1), 14-23.

Jones, H. N., Woollett, L. A., Barbour, N., Prasad, P. D., Powell, T. L., \& Jansson, T. (2009). High-fat diet before and during pregnancy causes marked up-regulation of placental nutrient transport and fetal overgrowth in C57/BL6 mice. FASEB J, 23(1), 271-278. doi: fj.08-116889 [pii]

10.1096/fj.08-116889

Jones, L. C., \& Clark, A. (2001). beta-cell neogenesis in type 2 diabetes. Diabetes, 50 Suppl 1, S186-187.

Jonsson, J., Carlsson, L., Edlund, T., \& Edlund, H. (1994). Insulin-promoter-factor 1 is required for pancreas development in mice. Nature, 371(6498), 606-609.

Jovanovic-Peterson, L., Fuhrmann, K., Hedden, K., Walker, L., \& Peterson, C. M. (1989). Maternal milk and plasma glucose and insulin levels: studies in normal and diabetic subjects. J Am Coll Nutr, 8(2), 125-131.

Jurgens, C. A., Toukatly, M. N., Fligner, C. L., Udayasankar, J., Subramanian, S. L., Zraika, S., et al. (2011). beta-cell loss and beta-cell apoptosis in human type 2 diabetes are related to islet amyloid deposition. Am J Pathol, 178(6), 2632-2640. doi: S0002-9440(11)00270-7 [pii]

10.1016/j.ajpath.2011.02.036

Kahn, B. B., \& Flier, J. S. (2000). Obesity and insulin resistance. J Clin Invest, 106(4), 473-481. doi: 10.1172/JCI10842

Kahn, R., Buse, J., Ferrannini, E., \& Stern, M. (2005). The metabolic syndrome: time for a critical appraisal. Joint statement from the American Diabetes Association and the European Association for the Study of Diabetes. Diabetologia, 48(9), 16841699. doi: 10.1007/s00125-005-1876-2 
Kashyap, S., Belfort, R., Gastaldelli, A., Pratipanawatr, T., Berria, R., Pratipanawatr, W., et al. (2003). A sustained increase in plasma free fatty acids impairs insulin secretion in nondiabetic subjects genetically predisposed to develop type 2 diabetes. Diabetes, 52(10), 2461-2474.

Kaufman, D., Smith, E. L., Gohil, B. C., Banerji, M., Coplan, J. D., Kral, J. G., et al. (2005). Early appearance of the metabolic syndrome in socially reared bonnet macaques. J Clin Endocrinol Metab, 90(1), 404-408. doi: jc.2004-0452 [pii]

10.1210/jc.2004-0452

Kerr, G. R., Allen, J. R., Scheffler, G., \& Couture, J. (1974). Fetal and postnatal growth of rhesus monkeys (M. mulatta). J Med Primatol, 3(4), 221-235.

Kidd, M., Nadler, B., Mane, S., Eick, G., Malfertheiner, M., Champaneria, M., et al. (2007). GeneChip, geNorm, and gastrointestinal tumors: novel reference genes for real-time PCR. Physiol Genomics, 30(3), 363-370.

Kieffer, T. J., Heller, R. S., Unson, C. G., Weir, G. C., \& Habener, J. F. (1996). Distribution of glucagon receptors on hormone-specific endocrine cells of rat pancreatic islets. Endocrinology, 137(11), 5119-5125.

Kilpatrick, E. D., \& Robertson, R. P. (1998). Differentiation between glucose-induced desensitization of insulin secretion and beta-cell exhaustion in the HIT-T15 cell line. Diabetes, 47(4), 606-611.

Kim, A., Miller, K., Jo, J., Kilimnik, G., Wojcik, P., \& Hara, M. (2009). Islet architecture: A comparative study. Islets, 1(2), 129-136. doi: 10.4161/isl.1.2.9480

Kiserud, T. (2005). Physiology of the fetal circulation. Semin Fetal Neonatal Med, 10(6), 493-503. doi: S1744-165X(05)00068-5 [pii]

10.1016/j.siny.2005.08.007

Konig, S., Vest, M., \& Stahl, M. (1978). Interrelation of maternal and foetal glucose and free fatty acids. The role of insulin and glucagon. Eur J Pediatr, 128(3), 187-195.

Konner, A. C., Janoschek, R., Plum, L., Jordan, S. D., Rother, E., Ma, X., et al. (2007). Insulin action in AgRP-expressing neurons is required for suppression of hepatic glucose production. Cell Metab, 5(6), 438-449. doi: S1550-4131(07)00131-3 [pii] 10.1016/j.cmet.2007.05.004

Koukkou, E., Ghosh, P., Lowy, C., \& Poston, L. (1998). Offspring of normal and diabetic rats fed saturated fat in pregnancy demonstrate vascular dysfunction. Circulation, 98(25), 2899-2904.

Koupil, I., \& Toivanen, P. (2008). Social and early-life determinants of overweight and obesity in 18-year-old Swedish men. Int J Obes (Lond), 32(1), 73-81.

Kramer, J., Moeller, E. L., Hachey, A., Mansfield, K. G., \& Wachtman, L. M. (2009). Differential expression of GLUT2 in pancreatic islets and kidneys of New and Old World nonhuman primates. Am J Physiol Regul Integr Comp Physiol, 296(3), R786-793. doi: 90694.2008 [pii]

10.1152/ajpregu.90694.2008

Lang, G., Goldstein, M. S., \& Levine, R. (1954). Influence of the liver on uptake of glucose by extrahepatic tissues. Am J Physiol, 177(3), 447-450.

Leung, Y. M., Ahmed, I., Sheu, L., Gao, X., Hara, M., Tsushima, R. G., et al. (2006). Insulin regulates islet alpha-cell function by reducing KATP channel sensitivity to 
adenosine 5'-triphosphate inhibition. Endocrinology, 147(5), 2155-2162. doi: en.2005-1249 [pii]

10.1210/en.2005-1249

Liang, Y., Najafi, H., \& Matschinsky, F. M. (1990). Glucose regulates glucokinase activity in cultured islets from rat pancreas. J Biol Chem, 265(28), 16863-16866.

Limesand, S. W., Jensen, J., Hutton, J. C., \& Hay, W. W., Jr. (2005). Diminished betacell replication contributes to reduced beta-cell mass in fetal sheep with intrauterine growth restriction. Am J Physiol Regul Integr Comp Physiol, 288(5), R1297-1305. doi: 00494.2004 [pii]

10.1152/ajpregu.00494.2004

Limesand, S. W., Rozance, P. J., Smith, D., \& Hay, W. W., Jr. (2007). Increased insulin sensitivity and maintenance of glucose utilization rates in fetal sheep with placental insufficiency and intrauterine growth restriction. Am J Physiol Endocrinol Metab, 293(6), E1716-1725. doi: 00459.2007 [pii]

10.1152/ajpendo.00459.2007

Limesand, S. W., Rozance, P. J., Zerbe, G. O., Hutton, J. C., \& Hay, W. W., Jr. (2006). Attenuated insulin release and storage in fetal sheep pancreatic islets with intrauterine growth restriction. Endocrinology, 147(3), 1488-1497. doi: en.20050900 [pii]

10.1210/en.2005-0900

Long, N. M., George, L. A., Uthlaut, A. B., Smith, D. T., Nijland, M. J., Nathanielsz, P. W., et al. (2010). Maternal obesity and increased nutrient intake before and during gestation in the ewe results in altered growth, adiposity, and glucose tolerance in adult offspring. J Anim Sci, 88(11), 3546-3553. doi: jas.2010-3083 [pii]

10.2527/jas.2010-3083

Ludmer, P. L., Selwyn, A. P., Shook, T. L., Wayne, R. R., Mudge, G. H., Alexander, R. W., et al. (1986). Paradoxical vasoconstriction induced by acetylcholine in atherosclerotic coronary arteries. $N$ Engl J Med, 315(17), 1046-1051. doi: 10.1056/NEJM198610233151702

Lukinius, A., Korsgren, O., Grimelius, L., \& Wilander, E. (1996). Expression of islet amyloid polypeptide in fetal and adult porcine and human pancreatic islet cells. Endocrinology, 137(12), 5319-5325.

MacDonald, P. E., Joseph, J. W., \& Rorsman, P. (2005). Glucose-sensing mechanisms in pancreatic beta-cells. Philos Trans R Soc Lond B Biol Sci, 360(1464), 2211-2225.

Maedler, K., \& Donath, M. Y. (2004). Beta-cells in type 2 diabetes: a loss of function and mass. Horm Res, 62 Suppl 3, 67-73. doi: 80503 [pii]

$10.1159 / 000080503$

Magarey, A. M., Daniels, L. A., Boulton, T. J., \& Cockington, R. A. (2003). Predicting obesity in early adulthood from childhood and parental obesity. Int J Obes Relat Metab Disord, 27(4), 505-513.

Martin-Gronert, M. S., \& Ozanne, S. E. (2007). Experimental IUGR and later diabetes. $J$ Intern Med, 261(5), 437-452. doi: JIM1800 [pii]

10.1111/j.1365-2796.2007.01800.x

Matschinsky, F. M. (1996). Banting Lecture 1995. A lesson in metabolic regulation inspired by the glucokinase glucose sensor paradigm. Diabetes, 45(2), 223-241.

Comstock, 155 
Matsumoto, S., Qualley, S. A., Goel, S., Hagman, D. K., Sweet, I. R., Poitout, V., et al. (2002). Effect of the two-layer (University of Wisconsin solutionperfluorochemical plus O2) method of pancreas preservation on human islet isolation, as assessed by the Edmonton Isolation Protocol. Transplantation, 74(10), 1414-1419. doi: 10.1097/01.TP.0000034206.66890.B0

Matthews, D. R., Hosker, J. P., Rudenski, A. S., Naylor, B. A., Treacher, D. F., \& Turner, R. C. (1985). Homeostasis model assessment: insulin resistance and beta-cell function from fasting plasma glucose and insulin concentrations in man.

Diabetologia, 28(7), 412-419.

Matveyenko, A. V., Singh, I., Shin, B. C., Georgia, S., \& Devaskar, S. U. (2010). Differential effects of prenatal and postnatal nutritional environment on beta-cell mass development and turnover in male and female rats. Endocrinology, 151(12), 5647-5656. doi: en.2010-0978 [pii]

10.1210/en.2010-0978

McAuley, K. A., Williams, S. M., Mann, J. I., Walker, R. J., Lewis-Barned, N. J., Temple, L. A., et al. (2001). Diagnosing insulin resistance in the general population. Diabetes Care, 24(3), 460-464.

McCurdy, C. E., Bishop, J. M., Williams, S. M., Grayson, B. E., Smith, M. S., Friedman, J. E., et al. (2009). Maternal high-fat diet triggers lipotoxicity in the fetal livers of nonhuman primates. J Clin Invest, 119(2), 323-335.

Medrikova, D., Jilkova, Z. M., Bardova, K., Janovska, P., Rossmeisl, M., \& Kopecky, J. (2011). Sex differences during the course of diet-induced obesity in mice: adipose tissue expandability and glycemic control. Int J Obes (Lond). doi: ijo201187 [pii] 10.1038/ijo.2011.87

Meyer, K. M., Koch, J. M., Ramadoss, J., Kling, P. J., \& Magness, R. R. (2010). Ovine surgical model of uterine space restriction: interactive effects of uterine anomalies and multifetal gestations on fetal and placental growth. Biol Reprod, 83(5), 799806. doi: biolreprod.110.085381 [pii]

10.1095/biolreprod.110.085381

Miki, T., Nagashima, K., \& Seino, S. (1999). The structure and function of the ATPsensitive $\mathrm{K}+$ channel in insulin-secreting pancreatic beta-cells. J Mol Endocrinol, 22(2), 113-123.

Mojsov, S., Weir, G. C., \& Habener, J. F. (1987). Insulinotropin: glucagon-like peptide I (7-37) co-encoded in the glucagon gene is a potent stimulator of insulin release in the perfused rat pancreas. J Clin Invest, 79(2), 616-619. doi: 10.1172/JCI112855

Montanya, E., \& Tellez, N. (2009). Pancreatic remodeling: beta-cell apoptosis, proliferation and neogenesis, and the measurement of beta-cell mass and of individual beta-cell size. Methods Mol Biol, 560, 137-158.

Moran, A., Zhang, H. J., Olson, L. K., Harmon, J. S., Poitout, V., \& Robertson, R. P. (1997). Differentiation of glucose toxicity from beta cell exhaustion during the evolution of defective insulin gene expression in the pancreatic islet cell line, HIT-T15. J Clin Invest, 99(3), 534-539. doi: 10.1172/JCI119190

Moritz, W., Leech, C. A., Ferrer, J., \& Habener, J. F. (2001). Regulated expression of adenosine triphosphate-sensitive potassium channel subunits in pancreatic betacells. Endocrinology, 142(1), 129-138.

Comstock, 156 
Morrison, J. L. (2008). Sheep models of intrauterine growth restriction: fetal adaptations and consequences. Clin Exp Pharmacol Physiol, 35(7), 730-743. doi: CEP4975 [pii]

10.1111/j.1440-1681.2008.04975.x

Mourmeaux, J. L., Remacle, C., \& Henquin, J. C. (1985). Morphological and functional characteristics of islets neoformed during tissue culture of fetal rat pancreas. $\mathrm{Mol}$ Cell Endocrinol, 39(3), 237-246.

Myers, R. E., Hill, D. E., Holt, A. B., Scott, R. E., Mellits, E. D., \& Cheek, D. B. (1971). Fetal growth retardation produced by experimental placental insufficiency in the rhesus monkey. I. Body weight, organ size. Biol Neonate, 18(5), 379-394.

Newgard, C. B., \& McGarry, J. D. (1995). Metabolic coupling factors in pancreatic betacell signal transduction. Annu Rev Biochem, 64, 689-719.

Nolan, C. J., Madiraju, M. S., Delghingaro-Augusto, V., Peyot, M. L., \& Prentki, M. (2006). Fatty acid signaling in the beta-cell and insulin secretion. Diabetes, 55 Suppl 2, S16-23. doi: 55/Supplement_2/S16 [pii]

10.2337/diabetes.

Offield, M. F., Jetton, T. L., Labosky, P. A., Ray, M., Stein, R. W., Magnuson, M. A., et al. (1996). PDX-1 is required for pancreatic outgrowth and differentiation of the rostral duodenum. Development, 122(3), 983-995.

Ogden, C. L., Carroll, M. D., Curtin, L. R., Lamb, M. M., \& Flegal, K. M. (2010). Prevalence of high body mass index in US children and adolescents, 2007-2008. JAMA, 303(3), 242-249. doi: 2009.2012 [pii]

10.1001/jama.2009.2012

Ogden, C. L., Carroll, M. D., Curtin, L. R., McDowell, M. A., Tabak, C. J., \& Flegal, K. M. (2006). Prevalence of overweight and obesity in the United States, 1999-2004. Jama, 295(13), 1549-1555.

Olson, L. K., Sharma, A., Peshavaria, M., Wright, C. V., Towle, H. C., Rodertson, R. P., et al. (1995). Reduction of insulin gene transcription in HIT-T15 beta cells chronically exposed to a supraphysiologic glucose concentration is associated with loss of STF-1 transcription factor expression. Proc Natl Acad Sci U S A, 92(20), 9127-9131.

Oltjen, J. W., \& Beckett, J. L. (1996). Role of ruminant livestock in sustainable agricultural systems. J Anim Sci, 74(6), 1406-1409.

Park, J. H., Stoffers, D. A., Nicholls, R. D., \& Simmons, R. A. (2008). Development of type 2 diabetes following intrauterine growth retardation in rats is associated with progressive epigenetic silencing of Pdx1. J Clin Invest, 118(6), 2316-2324. doi: 10.1172/JCI33655

Parton, L. E., Ye, C. P., Coppari, R., Enriori, P. J., Choi, B., Zhang, C. Y., et al. (2007). Glucose sensing by POMC neurons regulates glucose homeostasis and is impaired in obesity. Nature, 449(7159), 228-232. doi: nature06098 [pii]

10.1038/nature06098

Paulsen, S. J., Jelsing, J., Madsen, A. N., Hansen, G., Lykkegaard, K., Larsen, L. K., et al. (2010). Characterization of beta-cell mass and insulin resistance in dietinduced obese and diet-resistant rats. Obesity (Silver Spring), 18(2), 266-273. doi: oby2009245 [pii] 
10.1038/oby.2009.245

Perfetti, R., Zhou, J., Doyle, M. E., \& Egan, J. M. (2000). Glucagon-like peptide-1 induces cell proliferation and pancreatic-duodenum homeobox-1 expression and increases endocrine cell mass in the pancreas of old, glucose-intolerant rats. Endocrinology, 141(12), 4600-4605.

Phillips, D. I. (1996). Insulin resistance as a programmed response to fetal undernutrition. Diabetologia, 39(9), 1119-1122.

Pipeleers, D. G., Schuit, F. C., Van Schravendijk, C. F., \& Van de Winkel, M. (1985). Interplay of nutrients and hormones in the regulation of glucagon release. Endocrinology, 117(3), 817-823.

Piper, K., Brickwood, S., Turnpenny, L. W., Cameron, I. T., Ball, S. G., Wilson, D. I., et al. (2004). Beta cell differentiation during early human pancreas development. $J$ Endocrinol, 181(1), 11-23.

Pocai, A., Lam, T. K., Gutierrez-Juarez, R., Obici, S., Schwartz, G. J., Bryan, J., et al. (2005). Hypothalamic K(ATP) channels control hepatic glucose production. Nature, 434(7036), 1026-1031. doi: nature03439 [pii]

10.1038/nature03439

Poitout, V. (2008). Glucolipotoxicity of the pancreatic beta-cell: myth or reality? Biochem Soc Trans, 36(Pt 5), 901-904. doi: BST0360901 [pii]

10.1042/BST0360901

Postic, C., Shiota, M., \& Magnuson, M. A. (2001). Cell-specific roles of glucokinase in glucose homeostasis. Recent Prog Horm Res, 56, 195-217.

Prado, C. L., Pugh-Bernard, A. E., Elghazi, L., Sosa-Pineda, B., \& Sussel, L. (2004). Ghrelin cells replace insulin-producing beta cells in two mouse models of pancreas development. Proc Natl Acad Sci U S A, 101(9), 2924-2929. doi: 10.1073/pnas.0308604100

0308604100 [pii]

Quesada, I., Tuduri, E., Ripoll, C., \& Nadal, A. (2008). Physiology of the pancreatic alpha-cell and glucagon secretion: role in glucose homeostasis and diabetes. $J$ Endocrinol, 199(1), 5-19. doi: JOE-08-0290 [pii]

10.1677/JOE-08-0290

Radaelli, T., Lepercq, J., Varastehpour, A., Basu, S., Catalano, P. M., \& Hauguel-De Mouzon, S. (2009). Differential regulation of genes for fetoplacental lipid pathways in pregnancy with gestational and type 1 diabetes mellitus. Am J Obstet Gynecol, 201(2), 209 e201-209 e210. doi: S0002-9378(09)00405-0 [pii]

10.1016/j.ajog.2009.04.019

Rao, S., Yajnik, C. S., Kanade, A., Fall, C. H., Margetts, B. M., Jackson, A. A., et al. (2001). Intake of micronutrient-rich foods in rural Indian mothers is associated with the size of their babies at birth: Pune Maternal Nutrition Study. J Nutr, 131(4), 1217-1224.

Ravelli, A. C., van der Meulen, J. H., Michels, R. P., Osmond, C., Barker, D. J., Hales, C. N., et al. (1998). Glucose tolerance in adults after prenatal exposure to famine. Lancet, 351(9097), 173-177. doi: S0140673697072449 [pii]

Reddy, K. G., Nair, R. N., Sheehan, H. M., \& Hodgson, J. M. (1994). Evidence that selective endothelial dysfunction may occur in the absence of angiographic or

Comstock, 158 
ultrasound atherosclerosis in patients with risk factors for atherosclerosis. $J \mathrm{Am}$ Coll Cardiol, 23(4), 833-843. doi: 0735-1097(94)90627-0 [pii]

Reers, C., Erbel, S., Esposito, I., Schmied, B., Buchler, M. W., Nawroth, P. P., et al. (2009). Impaired islet turnover in human donor pancreata with aging. Eur J Endocrinol, 160(2), 185-191. doi: EJE-08-0596 [pii]

10.1530/EJE-08-0596

Remacle, C., Dumortier, O., Bol, V., Goosse, K., Romanus, P., Theys, N., et al. (2007). Intrauterine programming of the endocrine pancreas. Diabetes Obes Metab, 9 Suppl 2, 196-209. doi: DOM790 [pii]

10.1111/j.1463-1326.2007.00790.x

Rivas-Carrillo, J. D., Okitsu, T., Tanaka, N., \& Kobayashi, N. (2007). Pancreas development and beta-cell differentiation of embryonic stem cells. Curr Med Chem, 14(14), 1573-1578.

Roberts, V. H., Rasanen, J. P., Novy, M. J., Frias, A., Louey, S., Morgan, T. K., et al. (2012). Restriction of placental vasculature in a non-human primate: A unique model to study placental plasticity. Placenta, 33(1), 73-76. doi: S01434004(11)00494-2 [pii]

10.1016/j.placenta.2011.10.003

Robertson, R. P. (2004). Chronic oxidative stress as a central mechanism for glucose toxicity in pancreatic islet beta cells in diabetes. J Biol Chem, 279(41), 4235142354. doi: 10.1074/jbc.R400019200

R400019200 [pii]

Roseboom, T., de Rooij, S., \& Painter, R. (2006). The Dutch famine and its long-term consequences for adult health. Early Hum Dev, 82(8), 485-491. doi: S03783782(06)00184-8 [pii]

10.1016/j.earlhumdev.2006.07.001

Roseboom, T. J., van der Meulen, J. H., Osmond, C., Barker, D. J., Ravelli, A. C., Schroeder-Tanka, J. M., et al. (2000). Coronary heart disease after prenatal exposure to the Dutch famine, 1944-45. Heart, 84(6), 595-598.

Rosenberg, L., Lipsett, M., Yoon, J. W., Prentki, M., Wang, R., Jun, H. S., et al. (2004). A pentadecapeptide fragment of islet neogenesis-associated protein increases beta-cell mass and reverses diabetes in C57BL/6J mice. Ann Surg, 240(5), 875884. doi: 00000658-200411000-00021 [pii]

Rozance, P. J., Limesand, S. W., \& Hay, W. W., Jr. (2006). Decreased nutrientstimulated insulin secretion in chronically hypoglycemic late-gestation fetal sheep is due to an intrinsic islet defect. Am J Physiol Endocrinol Metab, 291(2), E404411. doi: 00643.2005 [pii]

10.1152/ajpendo.00643.2005

Sanz Sampelayo, M. R., Chilliard, Y., Schmidely, P., \& Boza, J. (2007). Influence of type of diet on the fat constituents of goat and sheep milk. Small Ruminant Research, 68(1-2), 42-63. doi: 10.1016/j.smallrumres.2006.09.017

Sato, Y., Ito, T., Udaka, N., Kanisawa, M., Noguchi, Y., Cushman, S. W., et al. (1996). Immunohistochemical localization of facilitated-diffusion glucose transporters in rat pancreatic islets. Tissue Cell, 28(6), 637-643. 
Scaglia, L., Cahill, C. J., Finegood, D. T., \& Bonner-Weir, S. (1997). Apoptosis participates in the remodeling of the endocrine pancreas in the neonatal rat. Endocrinology, 138(4), 1736-1741.

Scholzen, T., \& Gerdes, J. (2000). The Ki-67 protein: from the known and the unknown. J Cell Physiol, 182(3), 311-322. doi: 10.1002/(SICI)10974652(200003)182:3<311::AID-JCP1>3.0.CO;2-9 [pii]

10.1002/(SICI)1097-4652(200003)182:3<311::AID-JCP1>3.0.CO;2-9

Sebert, S. P., Dellschaft, N. S., Chan, L. L., Street, H., Henry, M., Francois, C., et al. (2011). Maternal nutrient restriction during late gestation and early postnatal growth in sheep differentially reset the control of energy metabolism in the gastric mucosa. Endocrinology, 152(7), 2816-2826. doi: en.2011-0169 [pii]

10.1210/en.2011-0169

Shafrir, E., \& Khassis, S. (1982). Maternal-fetal fat transport versus new fat synthesis in the pregnant diabetic rat. Diabetologia, 22(2), 111-117.

Sharma, A., Moore, M., Marcora, E., Lee, J. E., Qiu, Y., Samaras, S., et al. (1999). The NeuroD1/BETA2 sequences essential for insulin gene transcription colocalize with those necessary for neurogenesis and p300/CREB binding protein binding. Mol Cell Biol, 19(1), 704-713.

Sharma, A., Olson, L. K., Robertson, R. P., \& Stein, R. (1995). The reduction of insulin gene transcription in HIT-T15 beta cells chronically exposed to high glucose concentration is associated with the loss of RIPE3b1 and STF-1 transcription factor expression. Mol Endocrinol, 9(9), 1127-1134.

Slavin, B. G., Ong, J. M., \& Kern, P. A. (1994). Hormonal regulation of hormonesensitive lipase activity and mRNA levels in isolated rat adipocytes. $J$ Lipid Res, 35(9), 1535-1541.

Smith, C. A. (1947a). The effect of wartime starvation in Holland upon pregnancy and its product. Am J Obstet Gynecol, 53(4), 599-608.

Smith, C. A. (1947b). Effects of maternal under nutrition upon the newborn infant in Holland (1944-1945). J Pediatr, 30(3), 229-243.

Speakman, J., Hambly, C., Mitchell, S., \& Krol, E. (2007). Animal models of obesity. Obes Rev, 8 Suppl 1, 55-61.

Spruijt-Metz, D., Lindquist, C. H., Birch, L. L., Fisher, J. O., \& Goran, M. I. (2002). Relation between mothers' child-feeding practices and children's adiposity. Am J Clin Nutr, 75(3), 581-586.

Srinivasan, M., Aalinkeel, R., Song, F., Mitrani, P., Pandya, J. D., Strutt, B., et al. (2005). Maternal hyperinsulinemia predisposes rat fetuses for hyperinsulinemia and adult-onset obesity and maternal mild food restriction reverses this phenotype. Am J Physiol Endocrinol Metab.

Srinivasan, M., Katewa, S. D., Palaniyappan, A., Pandya, J. D., \& Patel, M. S. (2006). Maternal high-fat diet consumption results in fetal malprogramming predisposing to the onset of metabolic syndrome-like phenotype in adulthood. Am J Physiol Endocrinol Metab, 291(4), E792-799. doi: 00078.2006 [pii]

10.1152/ajpendo.00078.2006

Staub, A., Sinn, L., \& Behrens, O. K. (1955). Purification and crystallization of glucagon. J Biol Chem, 214(2), 619-632. 
Sullivan, E. L., Grayson, B., Takahashi, D., Robertson, N., Maier, A., Bethea, C. L., et al. Chronic consumption of a high-fat diet during pregnancy causes perturbations in the serotonergic system and increased anxiety-like behavior in nonhuman primate offspring. J Neurosci, 30(10), 3826-3830.

Sullivan, E. L., Grayson, B., Takahashi, D., Robertson, N., Maier, A., Bethea, C. L., et al. (2010). Chronic consumption of a high-fat diet during pregnancy causes perturbations in the serotonergic system and increased anxiety-like behavior in nonhuman primate offspring. J Neurosci, 30(10), 3826-3830. doi: 30/10/3826 [pii]

10.1523/JNEUROSCI.5560-09.2010

Taylor, P. D., McConnell, J., Khan, I. Y., Holemans, K., Lawrence, K. M., Asare-Anane, H., et al. (2005). Impaired glucose homeostasis and mitochondrial abnormalities in offspring of rats fed a fat-rich diet in pregnancy. Am J Physiol Regul Integr Comp Physiol, 288(1), R134-139. doi: 10.1152/ajpregu.00355.2004

00355.2004 [pii]

Teta, M., Rankin, M. M., Long, S. Y., Stein, G. M., \& Kushner, J. A. (2007). Growth and regeneration of adult beta cells does not involve specialized progenitors. Dev Cell, 12(5), 817-826. doi: S1534-5807(07)00158-X [pii]

10.1016/j.devcel.2007.04.011

Tomita, T., Masuzaki, H., Iwakura, H., Fujikura, J., Noguchi, M., Tanaka, T., et al. (2006). Expression of the gene for a membrane-bound fatty acid receptor in the pancreas and islet cell tumours in humans: evidence for GPR40 expression in pancreatic beta cells and implications for insulin secretion. Diabetologia, 49(5), 962-968. doi: 10.1007/s00125-006-0193-8

Uauy, R., Peirano, P., Hoffman, D., Mena, P., Birch, D., \& Birch, E. (1996). Role of essential fatty acids in the function of the developing nervous system. Lipids, 31 Suppl, S167-176.

Uchida, T., Nakamura, T., Hashimoto, N., Matsuda, T., Kotani, K., Sakaue, H., et al. (2005). Deletion of Cdkn1b ameliorates hyperglycemia by maintaining compensatory hyperinsulinemia in diabetic mice. Nat Med, 11(2), 175-182.

Uehara, S., Muroyama, A., Echigo, N., Morimoto, R., Otsuka, M., Yatsushiro, S., et al. (2004). Metabotropic glutamate receptor type 4 is involved in autoinhibitory cascade for glucagon secretion by alpha-cells of islet of Langerhans. Diabetes, 53(4), 998-1006.

Unger, R. H. (1995). Lipotoxicity in the pathogenesis of obesity-dependent NIDDM. Genetic and clinical implications. Diabetes, 44(8), 863-870.

Unger, R. H., \& Zhou, Y. T. (2001). Lipotoxicity of beta-cells in obesity and in other causes of fatty acid spillover. Diabetes, 50 Suppl 1, S118-121.

Van Assche, F. A., De Prins, F., Aerts, L., \& Verjans, M. (1977). The endocrine pancreas in small-for-dates infants. Br J Obstet Gynaecol, 84(10), 751-753.

van den Hoek, A. M., Voshol, P. J., Karnekamp, B. N., Buijs, R. M., Romijn, J. A., Havekes, L. M., et al. (2004). Intracerebroventricular neuropeptide Y infusion precludes inhibition of glucose and VLDL production by insulin. Diabetes, 53(10), 2529-2534. doi: 53/10/2529 [pii]

Comstock, 161 
Varastehpour, A., Radaelli, T., Minium, J., Ortega, H., Herrera, E., Catalano, P., et al. (2006). Activation of phospholipase A2 is associated with generation of placental lipid signals and fetal obesity. J Clin Endocrinol Metab, 91(1), 248-255. doi: jc.2005-0873 [pii]

$10.1210 /$ jc. $2005-0873$

Villar, J., Merialdi, M., Gulmezoglu, A. M., Abalos, E., Carroli, G., Kulier, R., et al. (2003). Nutritional interventions during pregnancy for the prevention or treatment of maternal morbidity and preterm delivery: an overview of randomized controlled trials. J Nutr, 133(5 Suppl 2), 1606S-1625S.

Wagner, J. D., Cline, J. M., Shadoan, M. K., Bullock, B. C., Rankin, S. E., \& Cefalu, W. T. (2001). Naturally occurring and experimental diabetes in cynomolgus monkeys: a comparison of carbohydrate and lipid metabolism and islet pathology. Toxicol Pathol, 29(1), 142-148.

Walker, J. N., Ramracheya, R., Zhang, Q., Johnson, P. R., Braun, M., \& Rorsman, P. (2011). Regulation of glucagon secretion by glucose: paracrine, intrinsic or both? Diabetes Obes Metab, 13 Suppl 1, 95-105. doi: 10.1111/j.14631326.2011.01450.x

Wang, X., Cahill, C. M., Pineyro, M. A., Zhou, J., Doyle, M. E., \& Egan, J. M. (1999). Glucagon-like peptide-1 regulates the beta cell transcription factor, PDX-1, in insulinoma cells. Endocrinology, 140(10), 4904-4907.

Weir, G. C., \& Bonner-Weir, S. (2004). Five stages of evolving beta-cell dysfunction during progression to diabetes. Diabetes, 53 Suppl 3, S16-21.

Weir, G. C., Marselli, L., Marchetti, P., Katsuta, H., Jung, M. H., \& Bonner-Weir, S. (2009). Towards better understanding of the contributions of overwork and glucotoxicity to the beta-cell inadequacy of type 2 diabetes. Diabetes Obes Metab, 11 Suppl 4, 82-90.

Weiss, R., Dziura, J., Burgert, T. S., Tamborlane, W. V., Taksali, S. E., Yeckel, C. W., et al. (2004). Obesity and the metabolic syndrome in children and adolescents. $N$ Engl J Med, 350(23), 2362-2374. doi: 10.1056/NEJMoa031049

350/23/2362 [pii]

Whitaker, R. C. (2004). Predicting preschooler obesity at birth: the role of maternal obesity in early pregnancy. Pediatrics, 114(1), e29-36.

Williams, J. A., \& Lee, M. (1976). Microtubules and pancreatic amylase release by mouse pancreas in vitro. J Cell Biol, 71(3), 795-806.

Wolfe-Coote, S., Louw, J., Woodroof, C., \& Du Toit, D. F. (1996). The non-human primate endocrine pancreas: development, regeneration potential and metaplasia. Cell Biol Int, 20(2), 95-101. doi: S1065-6995(96)90013-X [pii]

10.1006/cbir.1996.0013

Woods, S. C., Lotter, E. C., McKay, L. D., \& Porte, D., Jr. (1979). Chronic intracerebroventricular infusion of insulin reduces food intake and body weight of baboons. Nature, 282(5738), 503-505.

Xiao, X. Q., Williams, S. M., Grayson, B. E., Glavas, M. M., Cowley, M. A., Smith, M. S., et al. (2007). Excess weight gain during the early postnatal period is associated with permanent reprogramming of brown adipose tissue adaptive thermogenesis. Endocrinology, 148(9), 4150-4159. 
Yoon, J. C., Puigserver, P., Chen, G., Donovan, J., Wu, Z., Rhee, J., et al. (2001). Control of hepatic gluconeogenesis through the transcriptional coactivator PGC-1. Nature, 413(6852), 131-138. doi: 10.1038/35093050

35093050 [pii]

Zhang, L., Long, N. M., Hein, S. M., Ma, Y., Nathanielsz, P. W., \& Ford, S. P. (2011). Maternal obesity in ewes results in reduced fetal pancreatic beta-cell numbers in late gestation and decreased circulating insulin concentration at term. Domest Anim Endocrinol, 40(1), 30-39. doi: S0739-7240(10)00097-4 [pii]

10.1016/j.domaniend.2010.08.004

Zhang, X., Zhang, R., Raab, S., Zheng, W., Wang, J., Liu, N., et al. (2011). Rhesus macaques develop metabolic syndrome with reversible vascular dysfunction responsive to pioglitazone. Circulation, 124(1), 77-86. doi:

CIRCULATIONAHA.110.990333 [pii]

10.1161/CIRCULATIONAHA.110.990333

Zhu, M. J., Han, B., Tong, J., Ma, C., Kimzey, J. M., Underwood, K. R., et al. (2008). AMP-activated protein kinase signalling pathways are down regulated and skeletal muscle development impaired in fetuses of obese, over-nourished sheep. J Physiol, 586(10), 2651-2664. doi: jphysiol.2007.149633 [pii]

10.1113/jphysiol.2007.149633

Zhu, M. J., Ma, Y., Long, N. M., Du, M., \& Ford, S. P. (2010). Maternal obesity markedly increases placental fatty acid transporter expression and fetal blood triglycerides at midgestation in the ewe. Am J Physiol Regul Integr Comp Physiol, 299(5), R1224-1231. doi: ajpregu.00309.2010 [pii]

10.1152/ajpregu.00309.2010

Zimmet, P. Z. (1992). Kelly West Lecture 1991. Challenges in diabetes epidemiology-from West to the rest. Diabetes Care, 15(2), 232-252. 